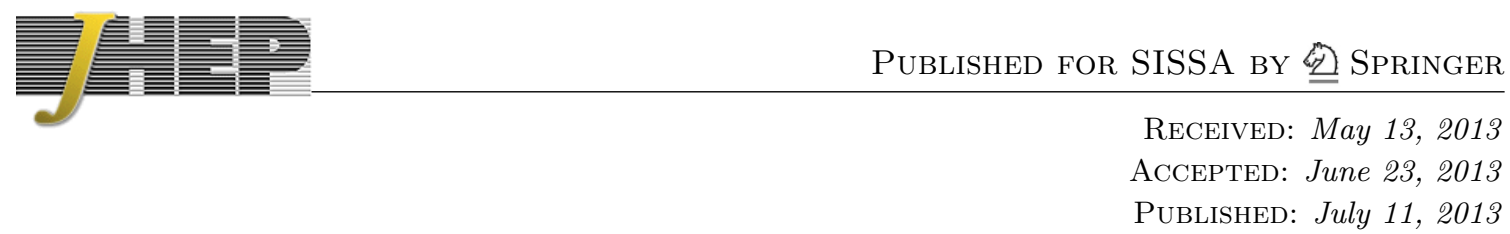

\title{
Heterotic non-abelian orbifolds
}

\author{
Maximilian Fischer, ${ }^{a}$ Saúl Ramos-Sánchez ${ }^{b}$ and Patrick K.S. Vaudrevange ${ }^{c}$ \\ ${ }^{a}$ Physik-Department T30, Technische Universität München, \\ James-Franck-Straße, 85748 Garching, Germany \\ ${ }^{b}$ Department of Theoretical Physics, Physics Institute, \\ UNAM, Mexico D.F. 04510, Mexico \\ ${ }^{c}$ Deutsches Elektronen-Synchrotron DESY, \\ Notkestraße 85, 22607 Hamburg, Germany \\ E-mail: maxi.fischer@mytum.de, ramos@fisica.unam.mx, \\ patrick.vaudrevange@desy.de
}

ABSTRACT: We perform the first systematic analysis of particle spectra obtained from heterotic string compactifications on non-Abelian toroidal orbifolds. After developing a new technique to compute the particle spectrum in the case of standard embedding based on higher dimensional supersymmetry, we compute the Hodge numbers for all recently classified 331 non-Abelian orbifold geometries which yield $\mathcal{N}=1$ supersymmetry for heterotic compactifications. Surprisingly, most Hodge numbers follow the empiric pattern $h^{(1,1)}-h^{(2,1)}=0 \bmod 6$, which might be related to the number of three standard model generations. Furthermore, we study the fundamental groups in order to identify the possibilities for non-local gauge symmetry breaking. Three examples are discussed in detail: the simplest non-Abelian orbifold $S_{3}$ and two more elaborate examples, $T_{7}$ and $\Delta(27)$, which have only one untwisted Kähler and no untwisted complex structure modulus. Such models might be especially interesting in the context of no-scale supergravity. Finally, we briefly discuss the case of orbifolds with vanishing Euler numbers in the context of enhanced (spontaneously broken) supersymmetry.

KEYWORDs: Flux compactifications, Superstrings and Heterotic Strings

ARXIV EPRINT: 1304.7742 


\section{Contents}

1 Introduction 1

2 Heterotic non-abelian orbifolds $\quad 2$

2.1 Hodge numbers 3

2.1.1 Contributions from the untwisted sectors 3

2.1.2 Contributions from the twisted sectors 5

2.2 Fundamental group 8

3 Examples $\quad 9$

$\begin{array}{lll}3.1 & \text { The heterotic } S_{3} \text { orbifold } & 10\end{array}$

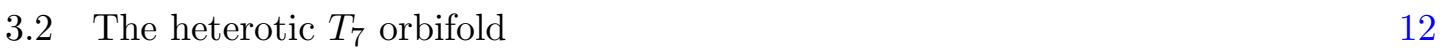

$\begin{array}{ll}3.3 \text { The heterotic } \Delta(27) \text { orbifold } & 14\end{array}$

4 Summary and discussion $\quad 16$

$\begin{array}{ll}\text { A Results } & 18\end{array}$

\section{Introduction}

Ten-dimensional superstring theory is perhaps the most promising candidate to yield an ultraviolet completion of particle physics and to explain some persisting cosmological puzzles. One useful mechanism to overcome the challenge of reducing the number of dimensions while preserving $\mathcal{N}=1$ supersymmetry and other phenomenologically appealing features is to compactify the six extra spatial dimensions on a toroidal orbifold.

Toroidal orbifolds offer a fairly simple geometrical structure that allows one to deal with the compactification in terms of the conformal-field framework of string theory [1,2]. Moreover, in recent years these constructions have become a fruitful source of semi-realistic models in the heterotic strings. ${ }^{1}$ The resulting scenarios can simultaneously reproduce the matter spectrum of the minimal supersymmetric version of the standard model [15-18] (or its singlet extensions [19]) and provide new approaches for solving puzzles such as the existence of hierarchies [20, 21], family symmetries [22] and proton stability [23, 24].

Despite these encouraging features, not all possible toroidal orbifold geometries have been explored. In the past, several efforts have led to partial classifications of orbifold geometries. The first attempts were restricted to $\mathbb{Z}_{N}$ orbifolds [25, 26]. Much later, $\mathbb{Z}_{2} \times \mathbb{Z}_{2}$

\footnotetext{
${ }^{1}$ References for other successful constructions are for example (see also references therein): for the free fermionic construction [3], for Gepner constructions [4], for type II with D-branes [5-7], for M-theory on $G_{2}$ manifolds [8] and for Calabi-Yau spaces [9, 10]. For the connection between (singular) orbifolds and (smooth) Calabi-Yau compactifications, see e.g. [11-14].
} 
orbifolds including so-called roto-translations ${ }^{2}$ were successfully classified [28] (see also [29, 30]). However, it is evident that more general orbifolds, which include not only Abelian (i.e. $\mathbb{Z}_{N}$ and $\mathbb{Z}_{M} \times \mathbb{Z}_{N}$ ) but also non-Abelian point groups (for example, $S_{n}$ for $n=3,4$ and $D_{2 n}$ for $n=2,3$ ), could also lead to appealing physics. Only recently a full classification of all $\left(\right.$ symmetric $^{3}$ ) toroidal orbifold geometries which preserve $\mathcal{N}=1$ SUSY in the context of heterotic compactifications has been achieved [32].

Even though most of the $\mathcal{N}=1$ heterotic orbifolds of ref. [32] (331 out of 469) are based on the discrete action of non-Abelian point groups, the geometrical aspects and phenomenology of these non-Abelian orbifolds have been studied only in few cases [33, 34]. The purpose of this paper is to provide the first tools to address these questions.

With this goal in mind, after a brief description of orbifold compactifications of the heterotic string, we develop a technique to systematically determine all twisted sectors and their fixed points/tori of all toroidal orbifolds in section 2. Further, for the so-called standard embedding of the orbifold action into the gauge degrees of freedom, which leads to an $\mathrm{E}_{6}$ gauge group, we develop a technique based on supersymmetry in four and six dimensions to compute the number of $\mathbf{2 7}$ and $\overline{\mathbf{2 7}}$ matter representations. This provides, as is known, the Hodge numbers $h^{1,1}$ and $h^{2,1}$, respectively. Furthermore, we determine the fundamental groups of all non-Abelian orbifolds in order to identify the possibility of non-local GUT breaking [35-37]. Section 3 is devoted to a detailed study of three sample non-Abelian orbifolds, with point groups $S_{3}, T_{7}$ and $\Delta(27)$, which illustrate the main properties of these constructions. Finally, we discuss our findings in section 4. Our most important results are summarized in table 3 for a list of all non-trivial fundamental groups and table 2 for a list of Hodge numbers and their geometrical origin.

\section{Heterotic non-abelian orbifolds}

We consider compactifications of the ten-dimensional heterotic string on (symmetric) toroidal orbifolds $[1,2]$, where points on the six-dimensional torus $T^{6}$ are identified under the action of the so-called orbifolding group $G$, i.e.

$$
\mathbb{O}=T^{6} / G=\mathbb{R}^{6} / S \text {. }
$$

Equivalently, the orbifold $\mathbb{O}$ is defined as $\mathbb{R}^{6}$ with an identification of points under the action of the so-called space group $S$. An element of $S$ consists of a rotational part and a translation. In detail,

$$
g=(\vartheta, \lambda) \in S \quad \text { acts on } x \in \mathbb{R}^{6} \text { as } \quad g x=\vartheta x+\lambda,
$$

where $\lambda$ is a shift of $x$ in $\mathbb{R}^{6}$ and $\vartheta \in \mathrm{SU}(3) \subset \mathrm{SO}(6)$ a rotation. Then, $g x \sim x$ for all $g \in S$ is the equivalence relation that defines $\mathbb{O}$. The pure-translational elements of $S$ have the form $(\mathbb{1}, \lambda)$, where $\lambda$ can be expanded in terms of six basis vectors $e_{i}$ as $\lambda=n_{i} e_{i}$ with

\footnotetext{
${ }^{2}$ They are also known as shift orbifolds; see e.g. ref. [27] for type IIA string theory on shift $\mathbb{Z}_{2} \times \mathbb{Z}_{2}$ orientifolds.

${ }^{3}$ For a recent work on asymmetric orbifolds, see e.g. [31].
} 
integer coefficients $n_{i}$ and summation over $i=1, \ldots, 6$. Hence, the pure translations define a lattice $\Lambda$ and hereby the torus $T^{6}=\mathbb{R}^{6} / \Lambda$. On the other hand, for $\vartheta \neq \mathbb{1}$ there can additionally be elements $(\vartheta, \lambda) \in S$ with $\lambda \notin \Lambda$ (i.e. with fractional $n_{i}$ ), which are called roto-translations [36].

The rotational part $\vartheta$ of all elements $g=(\vartheta, \lambda) \in S$ forms a group, the so-called point group $P$. We further define the orbifolding group $G$ as the group generated by all $g=(\vartheta, \lambda) \in S$, where two elements that are related by a pure lattice translation are identified. Therefore, the orbifolding group $G$ is equivalent to the point group $P$ if no roto-translations are present.

The Abelian case is well studied, resulting in many phenomenological interesting models in, for example, $\mathbb{Z}_{6}$-II [15-18, 38], $\mathbb{Z}_{12}$-I [39], $\mathbb{Z}_{2} \times \mathbb{Z}_{2}$ [40] and $\mathbb{Z}_{2} \times \mathbb{Z}_{4}$ [41] (there were also earlier phenomenological studies in $\mathbb{Z}_{3}$ and $\mathbb{Z}_{7}$, e.g. [42, 43]). In this paper we deal with nonAbelian orbifolds, i.e. with orbifolds whose point groups $P$ are non-Abelian. We consider all inequivalent point groups (also known as $\mathbb{Q}$-classes), all inequivalent lattices (called $\mathbb{Z}$ classes) and all roto-translations (i.e. affine classes) ${ }^{4}$. We use these six-dimensional spaces to compactify the $10 \mathrm{D} \mathrm{E}_{8} \times \mathrm{E}_{8}^{\prime}$ heterotic string to four dimensions.

On heterotic orbifolds, there are two kinds of closed strings which contribute to the massless particle spectrum of the resulting four-dimensional effective theory: (i) untwisted strings that close already in flat $\mathbb{R}^{6}$, and (ii) twisted strings that close only on the orbifold due to a non-trivial rotation $\vartheta$ (and possibly a translation $\lambda$ ) in the respective boundary condition, e.g. a twisted string generated by $g=(\vartheta, \lambda)$ closes under the boundary condition $X(\tau, \sigma+2 \pi)=\vartheta X(\tau, \sigma)+2 \pi \lambda$ for the bosonic string coordinate. It follows that twisted strings are localized at the fixed points/fixed tori of the orbifold geometry. In the case of standard embedding (which is a specific choice of how the orbifold acts in the gauge degrees of freedom of the heterotic string), the matter spectrum consists of $\mathbf{2 7}-, \overline{\mathbf{2 7}}$-plets and singlets of the four-dimensional observable gauge group $\mathrm{E}_{6}$. As we describe in more detail in the next section, counting the numbers of the non-trivial representations of $\mathrm{E}_{6}$ allows us to compute the Hodge numbers of these heterotic compactifications, which is the primary purpose of this paper.

\subsection{Hodge numbers}

The Hodge numbers $\left(h^{(1,1)}, h^{(2,1)}\right)$ count the Kähler and complex structure moduli, respectively, which correspond to deformations (of size and shape) of the geometry. For the heterotic orbifolds under consideration, we can split these numbers into contributions from the untwisted sector and from the twisted sectors,

$$
\left(h^{(1,1)}, h^{(2,1)}\right)=\left(h_{\mathrm{U}}^{(1,1)}, h_{\mathrm{U}}^{(2,1)}\right)+\left(h_{\mathrm{T}}^{(1,1)}, h_{\mathrm{T}}^{(2,1)}\right),
$$

and compute them as we explain in the following.

\subsubsection{Contributions from the untwisted sectors}

In this section, we demonstrate that the number of untwisted moduli can be computed directly from the point group $P$ using representation theory of finite groups.

\footnotetext{
${ }^{4}$ For further details on the definitions of these classes, we suggest refs. [32, 44, 45].
} 
The 4D untwisted Kähler and complex structure moduli, counted respectively by $h_{\mathrm{U}}^{(1,1)}$ and $h_{\mathrm{U}}^{(2,1)}$, originate from the nine plus nine internal components of the 10D supergravity multiplet of the heterotic string, which correspond to the following string excitations

$$
\begin{array}{ll}
|q\rangle_{\mathrm{R}} \otimes \tilde{\alpha}_{-1}^{\bar{j}}|0\rangle_{\mathrm{L}} & \text { for Kähler moduli, } \\
|q\rangle_{\mathrm{R}} \otimes \tilde{\alpha}_{-1}^{j}|0\rangle_{\mathrm{L}} & \text { for complex structure moduli, }
\end{array}
$$

where $j=1,2,3$ and $|q\rangle_{\mathrm{R}}$ denotes the ground state of the (supersymmetric) right-mover with (bosonized) momenta

$$
q=(0, \underline{-1,0,0}),
$$

where the underline denotes permutations. Furthermore, $\tilde{\alpha}_{-1}^{\bar{j}}$ or $\tilde{\alpha}_{-1}^{j}$ excites the left-moving ground state $|0\rangle_{\mathrm{L}}$ in the $j$-th complex plane spanned by the complex coordinate $\bar{z}^{\bar{j}}$ or $z^{j}$.

On the orbifold only the invariant combinations of these (untwisted) states survive as unfixed moduli. As untwisted moduli are uncharged with respect to the gauge group, they transform only under the action of the point group P. From table C.2 in ref. [32] we know the explicit form of the point group as a three-dimensional, in general reducible representation $\boldsymbol{\rho}$ of $P$, with $P$ being a finite sub-group of SU(3). Under the action of the point group $P$ the right-moving ground state and the oscillator excitations transform as

$$
\begin{array}{lll}
|q\rangle_{\mathrm{R}} & \text { transforms as } & \boldsymbol{\rho} \\
\tilde{\alpha}_{-1}^{\bar{j}} & \text { transforms as } & \overline{\boldsymbol{\rho}} \\
\tilde{\alpha}_{-1}^{j} & \text { transforms as } & \boldsymbol{\rho} .
\end{array}
$$

Hence, using equations (2.4) and (2.6), one can count the number of untwisted moduli $\left(h_{\mathrm{U}}^{(1,1)}, h_{\mathrm{U}}^{(2,1)}\right)$ from the tensor products

$$
\boldsymbol{\rho} \otimes \overline{\boldsymbol{\rho}} \rightarrow h_{\mathrm{U}}^{(1,1)} \boldsymbol{\rho}_{\mathbf{0}} \oplus \ldots \quad \text { and } \quad \boldsymbol{\rho} \otimes \boldsymbol{\rho} \rightarrow h_{\mathrm{U}}^{(2,1)} \boldsymbol{\rho}_{\mathbf{0}} \oplus \ldots,
$$

where $\rho_{\mathbf{0}}$ denotes the trivial singlet representation of $P$ and $h_{\mathrm{U}}^{(1,1)}$ and $h_{\mathrm{U}}^{(2,1)}$ are the multiplicities in the respective decomposition. These multiplicities can be computed most easily using characters (the character of an element $g \in P$ in the representation $\boldsymbol{\rho}$ is given by $\left.\chi_{\boldsymbol{\rho}}(g)=\operatorname{Tr}(\boldsymbol{\rho}(g))\right)$. In general, a decomposition of a tensor product reads $\boldsymbol{\rho}_{\mathbf{1}} \otimes \boldsymbol{\rho}_{\mathbf{2}}=\bigoplus_{i=1}^{c} n_{i} \boldsymbol{\rho}_{\boldsymbol{i}}$, where $c$ is the number of inequivalent irreducible representations, which are denoted as $\boldsymbol{\rho}_{\boldsymbol{i}}$, and $n_{i}$ are the corresponding multiplicities. Then, $\chi_{\boldsymbol{\rho}_{\mathbf{1}} \otimes \boldsymbol{\rho}_{\mathbf{2}}}(g)=\sum_{i=1}^{c} n_{i} \chi_{\boldsymbol{\rho}_{\boldsymbol{i}}}(g)$ and one can compute the multiplicities $n_{i}$ using the orthogonality of the rows of the character table, see e.g. section 4.2 of ref. [32].

We use the software GAP [46] and Mathematica to perform these computations. The results are listed in table 1 . Note that there are many cases with only one untwisted Kähler modulus (i.e. only the overall volume of $\mathbb{O}$ is unfixed) and no complex structure modulus (for example $P=T_{7}$, see section 3.2), which might be especially interesting in the context of no-scale supergravity [47-50]. This is in contrast to orbifolds with Abelian point groups where always at least the three Kähler moduli (associated with the sizes of the compact space split in three complex planes) survive the orbifold projection (see e.g. [51]). 


\begin{tabular}{|c|l|}
\hline $\begin{array}{c}\text { untwisted moduli } \\
\left(h_{\mathrm{U}}^{(1,1)}, h_{\mathrm{U}}^{(2,1)}\right)\end{array}$ & non-Abelian point groups \\
\hline$(2,2)$ & $S_{3}, D_{4}, D_{6}$ \\
\hline$(2,1)$ & $Q D_{16},\left(\mathbb{Z}_{4} \times \mathbb{Z}_{2}\right) \rtimes \mathbb{Z}_{2}, \mathbb{Z}_{4} \times S_{3},\left(\mathbb{Z}_{6} \times \mathbb{Z}_{2}\right) \rtimes \mathbb{Z}_{2}$, \\
& $\mathrm{GL}(2,3), \mathrm{SL}(2,3) \rtimes \mathbb{Z}_{2}$ \\
\hline$(2,0)$ & $\mathbb{Z}_{8} \rtimes \mathbb{Z}_{2}, \mathbb{Z}_{3} \times S_{3}, \mathbb{Z}_{3} \rtimes \mathbb{Z}_{8}, \mathrm{SL}(2,3)-\mathrm{I}, \mathbb{Z}_{3} \times D_{4}$, \\
& $\mathbb{Z}_{3} \times Q_{8},\left(\mathbb{Z}_{4} \times \mathbb{Z}_{4}\right) \rtimes \mathbb{Z}_{2}, \mathbb{Z}_{3} \times\left(\mathbb{Z}_{3} \rtimes \mathbb{Z}_{4}\right), \mathbb{Z}_{6} \times S_{3}$, \\
& $\mathbb{Z}_{3} \times \operatorname{SL}(2,3), \mathbb{Z}_{3} \times\left(\left(\mathbb{Z}_{6} \times \mathbb{Z}_{2}\right) \rtimes \mathbb{Z}_{2}\right), \operatorname{SL}(2,3) \rtimes \mathbb{Z}_{4}$ \\
\hline$(1,1)$ & $A_{4}, S_{4}$ \\
\hline$(1,0)$ & $T_{7}, \Delta(27), \mathbb{Z}_{3} \times A_{4}, \Delta(48), \Delta(54), \mathbb{Z}_{3} \times S_{4}, \Delta(96)$, \\
& $\Sigma(36 \phi), \Delta(108), \operatorname{PSL}(3,2), \Sigma(72 \phi), \Delta(216)$ \\
\hline
\end{tabular}

Table 1. List of non-Abelian point groups with specified number of untwisted moduli $\left(h_{\mathrm{U}}^{(1,1)}, h_{\mathrm{U}}^{(2,1)}\right)$. For example, orbifolds with point group $S_{3}, D_{4}$ and $D_{6}$ have two untwisted Kähler moduli and two untwisted complex structure moduli, i.e. $\left(h_{\mathrm{U}}^{(1,1)}, h_{\mathrm{U}}^{(2,1)}\right)=(2,2)$.

\subsubsection{Contributions from the twisted sectors}

The twisted sectors of the orbifold yield some twisted Kähler moduli (also known as blow-up modes) and twisted complex structure moduli (which describe the shapes of unorbifolded fixed tori, as explained in more detail later).

In order to determine their numbers, we analyze the standard embedding of the $\mathrm{E}_{8} \times \mathrm{E}_{8}^{\prime}$ heterotic string, which results in a four-dimensional $\mathcal{N}=1$ theory with $\mathrm{E}_{6} \times \mathrm{E}_{8}^{\prime}$ gauge group [34] (for Abelian point groups, the gauge group includes additional model-dependent gauge factors, such as $\mathrm{U}(1)^{2}, \mathrm{SU}(2) \times \mathrm{U}(1)$ or $\left.\mathrm{SU}(3)\right)$. Due to the $(2,2)$ world-sheet supersymmetry, the number of twisted 27-plets corresponds to $h_{\mathrm{T}}^{(1,1)}$ and the number of twisted $\overline{\mathbf{2 7}}$-plets gives $h_{\mathrm{T}}^{(2,1)}[52,53]$. In order to identify the number of twisted $\mathbf{2 7}$ - and $\overline{\mathbf{2 7}}$-plets we first have to consider the orbifold fixed points and fixed tori in some detail, with a special focus on four- and six-dimensional supersymmetry (see also ref. [54] for a related discussion).

Twisted sectors. In Abelian orbifolds, the twisted sectors are labeled by their point group elements, e.g. for a $\mathbb{Z}_{M} \times \mathbb{Z}_{N}$ point group with generators $\vartheta$ and $\omega$ we use $T_{k, \ell}$, with $k=0, \ldots, M-1$ and $\ell=0, \ldots, N-1$, to denote the (twisted) sector produced by $\vartheta^{k} \omega^{\ell} \in P$. In contrast, for non-Abelian orbifolds a twisted sector is characterized by a conjugation class $[\vartheta]$ for $\vartheta \in P$ and hence it is denoted as $T_{[\vartheta]}$.

Fixed points/tori. For a given twisted sector $T_{[\vartheta]}$, a space group element $g=(\vartheta, \lambda) \in S$ with $\vartheta \neq \mathbb{1}$ is called a constructing element of a massless string if the fixed point equation associated with $g$,

$$
g f=f \quad \Leftrightarrow \quad \vartheta f+\lambda=f \quad \text { for } f \in \mathbb{R}^{6},
$$

has a zero- or a two-dimensional solution $f$. In the former case, $f$ is called a fixed point, while in the latter it is called a fixed torus. 
Equivalence of fixed points/tori. Different solutions $f$ can be geometrically equivalent in the compact space due to the symmetries induced by the compactification. Equivalences between the solutions are easily identified via their corresponding constructing elements. We distinguish two different kinds of equivalences:

(i) Equivalence on the torus. Take two constructing elements of massless strings with the same point group element, i.e. $g_{1}=\left(\vartheta, \lambda_{1}\right) \in S$ and $g_{2}=\left(\vartheta, \lambda_{2}\right) \in S$. They are said to be equivalent on the torus if $g_{1}$ and $g_{2}$ are in the same conjugacy class with respect to translations, i.e.

$$
g_{1}=h g_{2} h^{-1} \quad \text { for some } h=(\mathbb{1}, \lambda) \in S .
$$

In other words, $g_{1} \sim g_{2}$ on the torus if $\lambda \in \Lambda$ exists such that $\lambda_{1}-\lambda_{2}=(\mathbb{1}-\vartheta) \lambda$. Then, the corresponding fixed points/tori differ by a lattice vector. Using this definition of equivalence one can determine for each twisted sector $T_{[\vartheta]}$ all inequivalent constructing elements on the torus.

(ii) Equivalence on the orbifold. Fixed points/tori that are inequivalent on the torus can be equivalent on the orbifold, i.e. fixed points/tori of a given twisted sector can be identified by a further orbifold action. Again, this equivalence can be determined using the concept of conjugacy classes, now allowing for general $h \in S$, i.e.

$$
g_{1} \sim g_{2} \quad \text { if } \quad g_{1}, g_{2} \in[g]=\left\{h g h^{-1} \text { for all } h \in S\right\} .
$$

Fixed points/tori associated with elements of the same conjugacy class are identified on the orbifold. In more detail, take $g_{1}, g_{2} \in[g]$ with

$$
g_{1} f_{1}=f_{1} \quad \text { and } \quad g_{2} f_{2}=f_{2},
$$

where $f_{1}, f_{2} \in \mathbb{R}^{6}$ denote the fixed points/tori. As $g_{1}, g_{2} \in[g]$ there exists an element $h \in S$ such that $g_{2}=h g_{1} h^{-1}$. Then, $\left(h^{-1} f_{2}\right)=\left(h^{-1} g_{2}\right) f_{2}=\left(g_{1} h^{-1}\right) f_{2}=$ $g_{1}\left(h^{-1} f_{2}\right)$. Consequently, $f_{1}=h^{-1} f_{2}$ and we see that the fixed points/tori $f_{1}$ and $f_{2}$ are identified on the orbifold.

Massless twisted matter. After obtaining all inequivalent constructing elements on the orbifold we start with the determination of the associated twisted matter spectrum. Each constructing element $g=(\vartheta, \lambda) \in S$ defines a boundary condition for a closed string on the orbifold, i.e.

$$
Z(\tau, \sigma+2 \pi)=g Z(\tau, \sigma)=\vartheta Z(\tau, \sigma)+2 \pi \lambda .
$$

For each twisted sector one can choose a basis of the three compactified complex coordinates $Z^{i}$ such that the twist $\vartheta \in \mathrm{SU}(3)$ becomes diagonal. Using this basis, the twist can be expressed by the so-called twist vector $v=\left(v_{1}, v_{2}, v_{3}\right)$, whose components are the rotational phases in units of $2 \pi$. These twist vectors are analogous to the well-known ones for the case of Abelian point groups (see e.g. table 1 in ref. [2] and table 5.2 in ref. [32]). Furthermore, the gauge embedding can be diagonalized such that its action is 
parametrized by a shift, similarly as in the Abelian case. For the standard embedding, we choose $V=\left(v_{1}, v_{2}, v_{3}, 0^{5}\right)\left(0^{8}\right)$. In order to compute the twisted matter, one may need a basis change for each twisted sector of a non-Abelian orbifold. However, for each individual sector one can use the standard tools and the intuition developed from the wellknown Abelian case, such as the usual masslessness equations for left- and right-movers and their solutions.

Invariance of twisted matter. In this way one can construct the Hilbert space $\mathcal{H}_{[g]}$ of massless twisted strings with constructing element $g$. However, not all states from $\mathcal{H}_{[g]}$ are necessarily invariant under the full orbifold action. One has to consider projections, i.e. one has to project the Hilbert space $\mathcal{H}_{[g]}$ of massless strings to the invariant subspace with respect to all space group elements $h$ that commute with the constructing element $g$, $g h=h g$. The set of commuting elements is called the centralizer of $g$. It is important to note that the rotational part of $g$ and $h$ and their gauge embeddings can be diagonalized simultaneously, as they commute.

For each constructing element $g \in[g]$ one distinguishes two cases:

1. In the first case, $g$ is related to a fixed point (not a fixed torus). Then, ten-dimensional $\mathcal{N}=1$ supersymmetry is broken down to $\mathcal{N}=1$ in four dimensions at the fixed point of $g$, and the Hilbert space $\mathcal{H}_{[g]}$ only respects $4 \mathrm{D} \mathcal{N}=1$. Fixed points with these properties contribute only one twisted $\mathbf{2 7}$-plet, which can be related to one twisted Kähler modulus (i.e. blow-up mode), but no $\overline{\mathbf{2 7}}$-plet and therefore no twisted complex structure modulus. In other words, the constructing element $g$ yields a contribution $(1,0)$ to the Hodge numbers $\left(h_{\mathrm{T}}^{(1,1)}, h_{\mathrm{T}}^{(2,1)}\right)$. Let us point out that $\mathcal{H}_{[g]}$ and $\mathcal{H}_{\left[g^{-1}\right]}$ are not independent, since $\mathcal{H}_{\left[g^{-1}\right]}$ contains the CPT conjugate partners of $\mathcal{H}_{[g]}$. Thus, it suffices to consider only $\mathcal{H}_{[g]}$ in the computations.

2. In the second case, $g$ has a fixed torus. Considering only the action of $g$ (and $g^{-1}$ ) on the internal space, the theory on this fixed torus has $\mathcal{N}=1$ in six dimensions (i.e. $4 \mathrm{D} \mathcal{N}=2$ ) with $\mathrm{E}_{7}$ observable gauge group and a twisted $\mathbf{5 6}$ hypermultiplet (or half-hypermultiplet). In terms of $4 \mathrm{D} \mathcal{N}=1$ this twisted 56-plet originates from the sector $g$ contributing a left-chiral superfield, which transforms as $\mathbf{5 6}$ of $\mathrm{E}_{7}$, and from the sector $g^{-1}$ contributing another left-chiral superfield, which transforms in the complex conjugate representation, e.g. as a $5 \mathbf{6}$-plet with negative $\mathrm{U}(1)$ charge. However, in the case $g=g^{-1}$ (or $[g]=\left[g^{-1}\right]$ ) the twisted 56-plet is real, e.g. a 56-plet with zero U(1) charge. Hence, it transforms as a half-hypermultiplet.

From the full $4 \mathrm{D}$ perspective the $\mathrm{E}_{7}$ is broken to $\mathrm{E}_{6}$ and the left-chiral 56-plet from $g$ branches into $\mathbf{2 7} \oplus \overline{\mathbf{2 7}}$ plus two singlets. Thus, 4D matter originates from $\mathcal{N}=2$ (half-)hypermultiplets and in terms of $4 \mathrm{D} \mathcal{N}=1$ a constructing element $g$ with fixed torus contributes both, one twisted 27-plet and one twisted $\overline{\mathbf{2 7}}$-plet, to the Hilbert space $\mathcal{H}_{[g]}$. This would result in one twisted Kähler modulus and one twisted complex structure modulus per fixed torus.

However, in the whole orbifold one has to perform the projection on invariant states: if there is (at least) one element in the centralizer of $g$ which breaks $\mathcal{N}=1$ in six 
dimensions to $\mathcal{N}=1$ in four dimensions, the twisted $\overline{\mathbf{2 7}}$-plet is removed from $\mathcal{H}_{[g]}$ and, consequently, the twisted complex structure modulus of this orbifolded fixed torus is projected out. Then, the fixed torus of $g$ contributes $(1,0)$ to the Hodge numbers. On the other hand, if all elements of the centralizer keep $\mathcal{N}=1$ in six dimensions, the twisted $\mathbf{2 7}$ - and $\overline{\mathbf{2 7}}$-plet and hence the respective moduli survive this projection. In this case, the fixed torus is not orbifolded further by the action of the centralizer and the twisted complex structure modulus describes the shape of this torus. Then, the fixed torus of $g$ contributes $(1,1)$ to the Hodge numbers.

Based on these observations, we notice that it is enough to know the geometrical aspects (space group, constructing elements, etc.) of the orbifold and not the details of the gauge embedding in order to arrive at the Hodge numbers. As a test, we have first used this procedure to corroborate the Hodge numbers for all 138 orbifolds with Abelian point groups of ref. [32] (originally obtained using the orbifolder [55]). Then, we applied this procedure to the 331 orbifolds with non-Abelian point groups. The results are listed in table 2 of appendix A. We discuss three examples in detail in section 3. It is interesting to note that, like in the Abelian case of ref. [32], also the Hodge numbers of most non-Abelian cases satisfy the empiric rule

$$
h^{(1,1)}-h^{(2,1)}=0 \bmod 6,
$$

for which we have not found an explanation yet (see also ref. [26]). In the cases where equation (2.13) is satisfied and the Euler number $\chi=2\left(h^{(1,1)}-h^{(2,1)}\right)$ does not vanish, it seems conceivable that the addition of discrete Wilson lines $[2,56]$ can lead to candidate models with three generations of standard model particles.

\section{$2.2 \quad$ Fundamental group}

The fundamental group $\pi_{1}$ of a toroidal orbifold is given by the following quotient group $[1,57]$

$$
\pi_{1}=S /\langle F\rangle,
$$

where $S$ is the space group that defines the orbifold, $F$ is the set of all constructing elements and $\langle F\rangle$ is the group generated by the elements of $F$.

There are two possible origins for a generator of $\pi_{1}$ : either it arises from a rototranslation (i.e. from the orbifolding group $G$ ) or from a pure translation (i.e. from the lattice $\Lambda$ ). In order to identify this, we compute in addition to $\pi_{1}=S /\langle F\rangle$ also $G / G_{F}$ and $\Lambda / \Lambda_{F}$, where $G_{F} \subset G$ is generated by the roto-translations of $\langle F\rangle$ and $\Lambda_{F} \subset \Lambda$ is the lattice of $\langle F\rangle$.

In total we find that 38 out of 331 orbifolds with non-Abelian point group and $\mathcal{N}=1$ have a non-trivial fundamental group, for example $\pi_{1}=\mathbb{Z}_{2}, \mathbb{Z}_{3}, \mathbb{Z}_{2} \times \mathbb{Z}_{2}$ and $\mathbb{Z}_{3} \times \mathbb{Z}_{3}$. They are listed in table 3 of appendix A. In the next section we discuss one of them in detail. Combined with the results of [32] we have a complete list of (toroidal, $\mathcal{N}=1$ ) orbifold geometries which offer a non-trivial fundamental group: there are 69 cases out of 469. These cases are of special interest for phenomenology as they may allow for non-local 
GUT breaking [35-37]. Therefore, the gauge embeddings of the (freely-acting) elements of the fundamental group and the conditions from modular invariance must be analyzed, cf. [11].

Example: $\boldsymbol{D}_{\mathbf{4}}$ orbifold. Let us discuss the case $D_{4}-1-5$ (i.e. $\mathbb{Z}$-class \#1, affine class \#5) with Hodge numbers $(6,6)$ in detail. $D_{4}$ is generated by $\vartheta$ and $\omega$ fulfilling $\vartheta^{2}=\omega^{2}=$ $(\vartheta \omega)^{4}=\mathbb{1}$. In the case (1-5) the space group $S$ is generated by $g_{1}=\left(\vartheta, \frac{1}{2} e_{1}+\frac{1}{4} e_{5}\right)$ and $g_{2}=(\omega, 0)$, where

$$
\vartheta_{\mathfrak{e}}=\left(\begin{array}{cccccc}
1 & 0 & 0 & 0 & 0 & 0 \\
0 & 0 & 0 & -1 & 0 & 0 \\
0 & 0 & -1 & 0 & 0 & 0 \\
0 & -1 & 0 & 0 & 0 & 0 \\
0 & 0 & 0 & 0 & -1 & 0 \\
0 & 0 & 0 & 0 & 0 & -1
\end{array}\right) \text { and } \omega_{\mathfrak{e}}=\left(\begin{array}{cccccc}
0 & 0 & 1 & 0 & 0 & 0 \\
0 & 1 & 0 & 0 & 0 & 0 \\
1 & 0 & 0 & 0 & 0 & 0 \\
0 & 0 & 0 & -1 & 0 & 0 \\
0 & 0 & 0 & 0 & -1 & 0 \\
0 & 0 & 0 & 0 & 0 & -1
\end{array}\right)
$$

and by the lattice $\Lambda=\left\{e_{1}, \ldots, e_{6}\right\}$.

On the other hand, the group $\langle F\rangle$ is generated by two roto-translations ${ }^{5}$

$$
h_{1}=(\omega, 0) \quad \text { and } \quad h_{2}=g_{1} g_{2} g_{1}=\left(\vartheta \omega \vartheta, \frac{1}{2}\left(e_{1}+e_{3}+e_{5}\right)\right)
$$

and six translations $\left(\mathbb{1}, e_{i}\right)$ for $i=2,4,5,6,\left(\mathbb{1}, e_{1}+e_{3}\right)$ and $\left(\mathbb{1},-e_{1}+e_{3}\right)$, which define a (six-dimensional) sublattice $\Lambda_{F} \subset \Lambda$.

As a subgroup of $G$ the roto-translations $h_{1}$ and $h_{2}$ generate $G_{F}=\mathbb{Z}_{2} \times \mathbb{Z}_{2}$ and one can show that $D_{4} /\left(\mathbb{Z}_{2} \times \mathbb{Z}_{2}\right)=\mathbb{Z}_{2}$, which is generated by $g_{1}$ using $g_{1}^{2}=\left(\mathbb{1}, e_{1}\right) \sim(\mathbb{1}, 0)$ in the orbifolding group. Furthermore, one can take the quotient of the respective lattices and obtains $\Lambda / \Lambda_{F}=\mathbb{Z}_{2}$, which is generated by $\left(\mathbb{1}, e_{3}\right)$ using $\left(\mathbb{1}, e_{3}\right)\left(\mathbb{1}, e_{3}\right)=\left(\mathbb{1}, 2 e_{3}\right) \sim(\mathbb{1}, 0)$ (or equivalently generated by $\left(\mathbb{1}, e_{1}\right)$ using $\left(\mathbb{1}, e_{1}\right)\left(\mathbb{1}, e_{1}\right)=\left(\mathbb{1}, 2 e_{1}\right) \sim(\mathbb{1}, 0)$ ).

The full fundamental group $\pi_{1}=S /\langle F\rangle$ of the orbifold $D_{4}-1-5$ is generated by $g_{1}$. Then, $g_{1}^{2}=\left(\mathbb{1}, e_{1}\right)$ (not identified with $(\mathbb{1}, 0)$ in $\left.\pi_{1}\right), g_{1}^{3}=\left(\vartheta, \frac{3}{2} e_{1}+\frac{1}{4} e_{5}\right)$ and $g_{1}^{4}=\left(\mathbb{1}, 2 e_{1}\right) \sim(\mathbb{1}, 0)$. Thus, we find $\pi_{1}=\mathbb{Z}_{4}$, see table 3 .

\section{$3 \quad$ Examples}

In this section we discuss three examples of orbifolds with non-Abelian point group in detail. The first example in section 3.1 considers $S_{3}$ [34], the easiest non-Abelian case, which unfortunately yields only non-chiral spectra. Then, in section 3.2 we discuss a $T_{7}$ orbifold which yields chirality. Furthermore, this model has the interesting property of having just one untwisted Kähler modulus and no untwisted complex structure modulus. Last, in section 3.3 we describe a $\Delta(27)$ orbifold which possesses a non-trivial fundamental group and gives chirality.

\footnotetext{
${ }^{5}$ Note that $\omega$ and $\vartheta \omega \vartheta$ belong to the same conjugacy class of $D_{4}$.
} 


\subsection{The heterotic $S_{3}$ orbifold}

The symmetric group $S_{3}$ is generated by two generators $\vartheta$ and $\omega$ of orders 2 and 3, i.e. $\vartheta^{2}=\omega^{3}=\mathbb{1}$. They fulfill the relation $\vartheta \omega \vartheta=\omega^{2} . S_{3}$ has $3 !=6$ elements which split into three conjugacy classes as follows:

$$
\begin{array}{ll}
{[\mathbb{1}]=\{\mathbb{1}\},} & v_{[\mathbb{1}]}=(0,0,0), \\
{[\omega]=\left\{\omega, \omega^{2}\right\},} & v_{[\omega]}=\left(\frac{1}{3},-\frac{1}{3}, 0\right), \\
{[\vartheta]=\left\{\vartheta, \vartheta \omega, \vartheta \omega^{2}\right\},} & v_{[\vartheta]}=\left(\frac{1}{2},-\frac{1}{2}, 0\right),
\end{array}
$$

where we listed for later use the corresponding twist vectors related to the corresponding $\mathrm{SU}(3)$-compatible point-group generators given below in equation (3.5) (obtained by choosing appropriate bases that diagonalize the respective rotation matrices).

From crystallography $[32,45]$, we know that for this Q-class (i.e. point group $P=S_{3}$ ), there are six $\mathbb{Z}$-classes (i.e. inequivalent lattices) and in total eleven affine classes (i.e. for each lattice except for lattice \#6 there are two affine classes: first the trivial affine class without roto-translations and a second affine class where $g_{\omega}$ is a roto-translation), see table 2 .

Let us discuss the first affine class, i.e. $S_{3}-1-1$. In this case the generators of the $S_{3}$ orbifolding group are $g_{\vartheta}=(\vartheta, 0)$ and $g_{\omega}=(\omega, 0)$, where

$$
\vartheta_{\mathfrak{e}}=\left(\begin{array}{cccccc}
1 & -1 & 0 & 0 & 0 & 0 \\
0 & -1 & 0 & 0 & 0 & 0 \\
0 & 0 & 0 & -1 & 0 & 0 \\
0 & 0 & -1 & 0 & 0 & 0 \\
0 & 0 & 0 & 0 & -1 & 0 \\
0 & 0 & 0 & 0 & 0 & -1
\end{array}\right) \quad \text { and } \quad \omega_{\mathfrak{e}}=\left(\begin{array}{cccccc}
-1 & 1 & 0 & 0 & 0 & 0 \\
-1 & 0 & 0 & 0 & 0 & 0 \\
0 & 0 & 0 & 1 & 0 & 0 \\
0 & 0 & -1 & -1 & 0 & 0 \\
0 & 0 & 0 & 0 & 1 & 0 \\
0 & 0 & 0 & 0 & 0 & 1
\end{array}\right) \text {, }
$$

given in the lattice basis as matrices from $\operatorname{GL}(6, \mathbb{Z})$, for example, $\vartheta_{\mathfrak{e}} e_{1}=e_{1}$. One can go to the $\mathrm{SO}(6)$ form by a basis change $\vartheta=B_{\mathfrak{e}} \vartheta_{\mathfrak{e}} B_{\mathfrak{e}}^{-1}$ and $\omega=B_{\mathfrak{e}} \omega_{\mathfrak{e}} B_{\mathfrak{e}}^{-1}$, where the columns of $B_{\mathfrak{e}}$ are the basis vectors $e_{i}, i=1, \ldots, 6$. In the $\mathrm{SU}(3)$ basis these generators read (see table C.2 of [32])

$$
\vartheta^{(3)}=\left(\begin{array}{ccc}
-1 & 0 & 0 \\
0 & 0 & 1 \\
0 & 1 & 0
\end{array}\right) \quad \text { and } \omega^{(3)}=\left(\begin{array}{ccc}
1 & 0 & 0 \\
0 \mathrm{e}^{-2 \pi \mathrm{i} \frac{1}{3}} & 0 \\
0 & 0 & \mathrm{e}^{2 \pi \mathrm{i} \frac{1}{3}}
\end{array}\right) .
$$

These matrices generate a reducible three-dimensional representation 3 of $S_{3}$, which decomposes into irreducible representations as $\mathbf{3}=\mathbf{2} \oplus \mathbf{1}^{\prime}$. Furthermore, there exists one additional irreducible representation of $S_{3}: \mathbf{1}$, the trivial singlet.

As discussed in section 2.1, the number of untwisted Kähler and complex structure moduli is determined by the tensor products of the three-dimensional representation of 
equation (3.5), i.e.

$$
\begin{aligned}
& \mathbf{3} \otimes \overline{\mathbf{3}}=\left(\mathbf{2} \oplus \mathbf{1}^{\prime}\right) \otimes\left(\overline{\mathbf{2}} \oplus \overline{\mathbf{1}^{\prime}}\right) \rightarrow \mathbf{2} \oplus \mathbf{2} \oplus \mathbf{2} \oplus \mathbf{1}^{\prime} \oplus \mathbf{1} \oplus \mathbf{1}, \\
& \mathbf{3} \otimes \mathbf{3}=\left(\mathbf{2} \oplus \mathbf{1}^{\prime}\right) \otimes\left(\mathbf{2} \oplus \mathbf{1}^{\prime}\right) \rightarrow \mathbf{2} \oplus \mathbf{2} \oplus \mathbf{2} \oplus \mathbf{1}^{\prime} \oplus \mathbf{1} \oplus \mathbf{1} .
\end{aligned}
$$

Since equation (3.6a) contains two trivial singlets 1, there are two orbifold-invariant untwisted Kähler moduli from the states given in equation (2.4a). Further, also equation (3.6b) contains two singlets $\mathbf{1}$ and hence there are also two orbifold-invariant untwisted complex structure moduli from equation (2.4b). In total, we find

$$
\left(h_{\mathrm{U}}^{(1,1)}, h_{\mathrm{U}}^{(2,1)}\right)=(2,2) .
$$

Next, we discuss the contributions from the two twisted sectors of the $S_{3}$ orbifold, specified by the inequivalent conjugacy classes given in equation (3.1). The $[\omega]$ twisted sector has nine inequivalent constructing elements $g^{(i)} \in S, i=1, \ldots, 9$ on the torus,

$$
\begin{array}{llll}
g^{(1)}=(\omega, 0) & g^{(2)}=\left(\omega, e_{4}\right) & , & g^{(3)}=\left(\omega, 2 e_{4}\right), \\
g^{(4)}=\left(\omega, e_{2}\right) & g^{(5)}=\left(\omega, e_{2}+e_{4}\right) & , & g^{(6)}=\left(\omega, e_{2}+2 e_{4}\right), \\
g^{(7)}=\left(\omega, e_{1}+e_{2}\right), & g^{(8)}=\left(\omega, e_{1}+e_{2}+e_{4}\right), & g^{(9)}=\left(\omega, e_{1}+e_{2}+2 e_{4}\right) .
\end{array}
$$

These constructing elements by themselves lead to a six-dimensional $\mathcal{N}=1$ supersymmetric theory, where the six dimensions include the uncompactified $4 \mathrm{D}$ space along with the twotorus defined by the basis vectors $e_{5}$ and $e_{6}$.

Finally, the $[\vartheta]$ sector has four inequivalent constructing elements on the torus,

$$
\left(\vartheta, n_{5} e_{5}+n_{6} e_{6}\right) \quad \text { with } \quad n_{5}, n_{6}=0,1
$$

which are also inequivalent on the orbifold. As the $[\omega]$ sector, the $[\vartheta]$ twisted sector yields an $\mathcal{N}=1$ supersymmetric theory in the six dimensions composed of the uncompactified $4 \mathrm{D}$ space and the two-torus defined by the basis vectors $e_{1}$ and $e_{4}-e_{3}$.

The centralizer elements of the constructing elements of both twisted sectors do not further break supersymmetry in their respective six-dimensional $\mathcal{N}=1$ theories. Therefore, all $9+4$ fixed tori are endowed with both a $\mathbf{2 7}$ - and a $\overline{\mathbf{2 7}}$-plet in four dimensions, contributing with as many twisted Kähler and complex-structure moduli as the number of inequivalent constructing elements.

In summary, the Hodge numbers are $\left(h^{(1,1)}, h^{(2,1)}\right)=(15,15)$ arising from the various sectors as

$$
(2,2) U+(9,9) T_{[\omega]}+(4,4) T_{[\vartheta]},
$$

confirming the results of [34]. Unfortunately, in the standard heterotic CFT description the $S_{3}$ orbifold necessarily leads to a non-chiral spectrum in $4 \mathrm{D}$, as we can see from the Hodge numbers $h^{(1,1)}=h^{(2,1)}$. Hence, the $S_{3}$ orbifold seems phenomenologically not promising. It might be possible to circumvent this by introducing magnetized tori [54]. 


\subsection{The heterotic $T_{7}$ orbifold}

The Frobenius group $T_{7}$ is generated by two generators $\vartheta$ and $\omega$ of orders 3 and 7 , i.e. $\vartheta^{3}=\omega^{7}=\mathbb{1}$. They fulfill the relation $\omega \vartheta=\vartheta \omega^{2} . T_{7}$ has 21 elements, they split into five conjugacy classes, i.e.

$$
\begin{aligned}
{[\mathbb{1}] } & =\{\mathbb{1}\}, & v_{[\mathbb{1}]} & =(0,0,0), \\
{[\omega] } & =\left\{\omega, \omega^{2}, \omega^{4}\right\}, & v_{[\omega]} & =\left(\frac{1}{7}, \frac{2}{7},-\frac{3}{7}\right), \\
{\left[\omega^{3}\right] } & =\left\{\omega^{3}, \omega^{5}, \omega^{6}\right\}, & v_{\left[\omega^{3}\right]} & =\left(-\frac{1}{7},-\frac{2}{7}, \frac{3}{7}\right), \\
{[\vartheta] } & =\left\{\vartheta, \vartheta \omega, \vartheta \omega^{2}, \vartheta \omega^{3}, \vartheta \omega^{4}, \vartheta \omega^{5}, \vartheta \omega^{6}\right\}, & v_{[\vartheta]} & =\left(\frac{1}{3},-\frac{1}{3}, 0\right), \\
{\left[\vartheta^{2}\right] } & =\left\{\vartheta^{2}, \vartheta^{2} \omega, \vartheta^{2} \omega^{2}, \vartheta^{2} \omega^{3}, \vartheta^{2} \omega^{4}, \vartheta^{2} \omega^{5}, \vartheta^{2} \omega^{6}\right\}, & v_{\left[\vartheta^{2}\right]} & =\left(\frac{1}{3},-\frac{1}{3}, 0\right) .
\end{aligned}
$$

where, as in the $S_{3}$ example, we provide for later use the corresponding twist vectors associated with equation (3.17).

From crystallography $[32,45]$ we know that for this $\mathbb{Q}$-class (i.e. point group $P=T_{7}$ ), there are three $\mathbb{Z}$-classes (i.e. inequivalent lattices) and in total three affine classes (i.e. for each lattice there is only the trivial affine class without roto-translations), see table 2 .

Let us discuss the first $\mathbb{Z}$-class, i.e. $T_{7}-1-1$. In this case the generators of the $T_{7}$ orbifolding group are $g_{\vartheta}=(\vartheta, 0)$ and $g_{\omega}=(\omega, 0)$, where

$$
\vartheta_{\mathfrak{e}}=\left(\begin{array}{cccccc}
0 & -1 & 0 & 0 & -1 & 0 \\
0 & -1 & 0 & 1 & -1 & 0 \\
0 & 1 & 0 & -1 & 1 & -1 \\
-1 & 0 & 0 & 0 & 0 & -1 \\
0 & 0 & -1 & 0 & 1 & 0 \\
1 & 0 & 0 & 0 & 1 & 0
\end{array}\right) \quad \text { and } \quad \omega_{\mathfrak{e}}=\left(\begin{array}{cccccc}
0 & -1 & 0 & 0 & 0 & -1 \\
1 & -1 & 0 & 0 & 0 & -1 \\
-1 & 1 & 0 & 0 & -1 & 1 \\
1 & 0 & 1 & 0 & -1 & 0 \\
-1 & 1 & 0 & 0 & 0 & 0 \\
-1 & 1 & 0 & -1 & 0 & 0
\end{array}\right)
$$

given in the lattice basis as matrices from $\mathrm{GL}(6, \mathbb{Z})$, for example, $\vartheta_{\mathfrak{e}} e_{1}=-e_{4}+e_{6}$. One can go to the $\mathrm{SO}(6)$ form by a basis change $\vartheta=B_{\mathfrak{e}} \vartheta_{\mathfrak{e}} B_{\mathfrak{e}}^{-1}$ and $\omega=B_{\mathfrak{e}} \omega_{\mathfrak{e}} B_{\mathfrak{e}}^{-1}$, where the columns of $B_{\mathfrak{e}}$ are the basis vectors $e_{i}, i=1, \ldots, 6$. In the $\mathrm{SU}(3)$ basis these generators read (see table C.2 of [32])

$$
\vartheta^{(3)}=\left(\begin{array}{lll}
0 & 1 & 0 \\
0 & 0 & 1 \\
1 & 0 & 0
\end{array}\right) \quad \text { and } \quad \omega^{(3)}=\left(\begin{array}{ccc}
\mathrm{e}^{2 \pi \mathrm{i} \frac{4}{7}} & 0 & 0 \\
0 & \mathrm{e}^{2 \pi \mathrm{i} \frac{2}{7}} & 0 \\
0 & 0 & \mathrm{e}^{2 \pi \mathrm{i} \frac{1}{7}}
\end{array}\right) \text {. }
$$

These matrices generate an irreducible three-dimensional representation 3 of $T_{7}$. Furthermore, there exist four additional irreducible representations of $T_{7}: \overline{\mathbf{3}}$ is the complex conjugate of $\mathbf{3}, \mathbf{1}^{\prime}$ and its complex conjugate $\overline{\mathbf{1}^{\prime}}$ are two non-trivial one-dimensional representations and, finally, $\mathbf{1}$ is the trivial singlet. 
One can think of the $T_{7}$ orbifold as a standard $\mathbb{Z}_{7}$ orbifold generated by $\omega$ with an additional, non-freely acting $\mathbb{Z}_{3}$ generated by $\vartheta$ that permutes the three complex planes $\left(z_{1}, z_{2}, z_{3}\right)$ as $z_{1} \mapsto z_{3} \mapsto z_{2} \mapsto z_{1}$.

As discussed in section 2.1, the number of untwisted Kähler and complex structure moduli is determined by the tensor products of the three-dimensional representation of equation (3.17), i.e.

$$
\begin{aligned}
& \mathbf{3} \otimes \overline{\mathbf{3}} \rightarrow \mathbf{3} \oplus \overline{\mathbf{3}} \oplus \mathbf{1} \oplus \mathbf{1}^{\prime} \oplus \overline{\mathbf{1}^{\prime}}, \\
& \mathbf{3} \otimes \mathbf{3} \rightarrow(\mathbf{3} \oplus \overline{\mathbf{3}})_{s} \oplus \overline{\mathbf{3}}_{a},
\end{aligned}
$$

where $s$ and $a$ denotes the symmetric and anti-symmetric part, respectively. As equation (3.18a) contains one singlet $\mathbf{1}$, there is one orbifold-invariant untwisted Kähler modulus from equation (2.4a). Furthermore, equation (3.18b) does not contain the singlet 1 and hence there is no orbifold-invariant untwisted complex structure modulus from equation (2.4b). In summary, we find

$$
\left(h_{\mathrm{U}}^{(1,1)}, h_{\mathrm{U}}^{(2,1)}\right)=(1,0) .
$$

Next, we study the contributions from the four twisted sectors of the $T_{7}$ orbifold arising from its conjugacy classes (see equation (3.11)). The $[\omega]$ twisted sector has seven inequivalent constructing elements $g^{(i)} \in S, i=1, \ldots, 7$, on the torus:

$$
\begin{array}{ll}
g^{(1)}=(\omega, 0) & , g^{(2)}=\left(\omega, e_{1}+e_{2}\right) \\
g^{(3)}=\left(\omega, e_{1}+e_{2}+e_{6}\right) & , g^{(4)}=\left(\omega, e_{1}+e_{3}+e_{5}+e_{6}\right), \\
g^{(5)}=\left(\omega, 2 e_{1}+2 e_{2}+e_{6}\right) & , g^{(6)}=\left(\omega, 2 e_{1}+e_{2}+e_{3}+e_{5}+e_{6}\right) \\
g^{(7)}=\left(\omega, 2 e_{1}+e_{2}+e_{3}+e_{5}+2 e_{6}\right) &
\end{array}
$$

They are also inequivalent on the orbifold. The corresponding fixed points are given in the $e_{\alpha}$ basis by $f^{(i)}=\frac{1}{7} \hat{f}_{\alpha}^{(i)} e_{\alpha}, i=1, \ldots, 7$, with

$$
\begin{array}{lll}
\hat{f}^{(1)}=(0,0,0,0,0,0) \quad, \hat{f}^{(2)}=(2,4,1,1,2,1), & \hat{f}^{(3)}=(1,2,4,4,1,4), \\
\hat{f}^{(4)}=(4,1,2,2,4,2), & \hat{f}^{(5)}=(3,6,5,5,3,5), & \hat{f}^{(6)}=(6,5,3,3,6,3), \\
\hat{f}^{(7)}=(5,3,6,6,5,6) . &
\end{array}
$$

As these are fixed points (and not tori) and the centralizers of $g^{i}$ are trivial, the $[\omega]$ twisted sector combines with the inverse twisted sector $\left[\omega^{6}\right]=\left[\omega^{3}\right]$ and gives seven left-chiral 27plets plus their CPT conjugate partners. Hence, this sector contributes with $(7,0)$ to the Hodge numbers.

The $[\vartheta]$ twisted sector has one inequivalent constructing element with associated fixed torus,

$$
(\vartheta, 0) \quad \text { with } f=\left(f_{1}, f_{2}, 0,-f_{1}+f_{2},-f_{1}-f_{2},-f_{2}\right),
$$

where the torus is parametrized by $f_{1}, f_{2} \in \mathbb{R}$. As the centralizer of this sector is trivial, the $[\vartheta]$ twisted sector feels the full $\mathcal{N}=1$ in six dimensions and hence contributes $(1,1)$ to the Hodge numbers. 
Finally, the $\left[\vartheta^{2}\right]$ is very similar to the $[\vartheta]$ twisted sector. It has one inequivalent constructing element with associated fixed torus,

$$
\left(\vartheta^{2}, 0\right) \text { with } f=\left(f_{1}, f_{2}, 0,-f_{1}+f_{2},-f_{1}-f_{2},-f_{2}\right),
$$

where the torus is parametrized by $f_{1}, f_{2} \in \mathbb{R}$. Again, as the centralizer is trivial, it gives rise to one twisted Kähler and one twisted complex structure modulus and therefore contributes $(1,1)$ to the Hodge numbers.

In summary, the Hodge numbers are $\left(h^{(1,1)}, h^{(2,1)}\right)=(10,2)$, distributed in the various sectors according to

$$
(1,0) U+(7,0) T_{[\omega]}+(1,1) T_{[\vartheta]}+(1,1) T_{\left[\vartheta^{2}\right]} .
$$

\subsection{The heterotic $\Delta(27)$ orbifold}

The group $\Delta(27)$ is generated by two generators $\vartheta$ and $\omega$ both of order 3, i.e. such that $\vartheta^{3}=\omega^{3}=\mathbb{1} . \Delta(27)$ has 27 elements, they split into the following eleven conjugacy classes

$$
\begin{array}{llll}
{[\mathbb{1 1}]} & =\{\mathbb{1}\}, & v_{[1]]} & =(0,0,0), \\
{[\omega]} & =\left\{\omega, \vartheta \omega \vartheta^{2}, \vartheta \omega^{2} \vartheta^{2} \omega^{2}\right\}, & v_{[\omega]} & =\left(\frac{1}{3},-\frac{1}{3}, 0\right), \\
{\left[\omega^{2}\right]} & =\left\{\omega^{2}, \vartheta \omega^{2} \vartheta^{2}, \vartheta \omega \vartheta^{2} \omega\right\}, & v_{\left[\omega^{2}\right]} & =\left(\frac{1}{3},-\frac{1}{3}, 0\right), \\
{[\vartheta]} & =\left\{\vartheta, \omega \vartheta \omega^{2}, \omega^{2} \vartheta \omega\right\}, & v_{[\vartheta]} & =\left(\frac{1}{3},-\frac{1}{3}, 0\right), \\
{\left[\vartheta^{2}\right]} & =\left\{\vartheta^{2}, \omega^{2} \vartheta^{2} \omega, \omega \vartheta^{2} \omega^{2}\right\}, & v_{\left[\vartheta^{2}\right]} & =\left(\frac{1}{3},-\frac{1}{3}, 0\right), \\
{[\omega \vartheta]} & =\left\{\omega \vartheta, \omega^{2} \vartheta \omega^{2}, \vartheta \omega\right\}, & v_{[\omega \vartheta]} & =\left(\frac{1}{3},-\frac{1}{3}, 0\right), \\
{\left[\omega \vartheta^{2}\right]} & =\left\{\omega \vartheta^{2}, \vartheta^{2} \omega, \omega^{2} \vartheta^{2} \omega^{2}\right\}, & v_{\left[\omega \vartheta^{2}\right]} & =\left(\frac{1}{3},-\frac{1}{3}, 0\right), \\
{\left[\omega^{2} \vartheta\right]} & =\left\{\omega^{2} \vartheta, \vartheta \omega^{2}, \omega \vartheta \omega\right\}, & v_{\left[\omega^{2} \vartheta\right]} & =\left(\frac{1}{3},-\frac{1}{3}, 0\right), \\
{\left[\omega^{2} \vartheta^{2}\right]} & =\left\{\omega^{2} \vartheta^{2}, \omega \vartheta^{2} \omega, \vartheta^{2} \omega^{2}\right\}, & v_{\left[\omega^{2} \vartheta^{2}\right]} & =\left(\frac{1}{3},-\frac{1}{3}, 0\right), \\
{\left[\vartheta \omega \vartheta^{2} \omega^{2}\right]} & =\left\{\vartheta \omega \vartheta^{2} \omega^{2}\right\}, & v_{\left[\vartheta \omega \vartheta^{2} \omega^{2}\right]} & =\left(\frac{1}{3}, \frac{1}{3},-\frac{2}{3}\right), \\
{\left[\vartheta \omega^{2} \vartheta^{2} \omega\right]} & =\left\{\vartheta \omega^{2} \vartheta^{2} \omega\right\}, & v_{\left[\vartheta \omega^{2} \vartheta^{2} \omega\right]} & =\left(\frac{1}{3}, \frac{1}{3},-\frac{2}{3}\right),
\end{array}
$$

where we also give the corresponding twist vectors obtained, as before, by choosing bases in which the rotation matrices are diagonal, as in equation (3.27).

Once again, it is known that there are three lattices and a total of ten affine classes (three orbifolding groups without roto-translations and seven ones which include them) for the point group $P=\Delta(27)$.

Let us discuss the fourth affine class of the first $\mathbb{Z}$-class, i.e. $\Delta(27)-1-4$, see table 2 . In this case the generators of the $\Delta(27)$ orbifolding group are $g_{\vartheta}=\left(\vartheta, \frac{1}{3}\left(2 e_{2}+e_{3}+2 e_{5}\right)\right)$ and $g_{\omega}=\left(\omega, \frac{1}{3} e_{1}\right)$, where

$$
\vartheta_{\mathfrak{e}}=\left(\begin{array}{cccccc}
0 & 1 & 0 & 0 & 1 & 0 \\
0 & 0 & 0 & 0 & 0 & 1 \\
0 & 1 & 0 & -1 & 2 & 1 \\
0 & 0 & 1 & -1 & 1 & 1 \\
0 & 0 & 0 & 0 & 1 & 0 \\
1 & 0 & 0 & 0 & -1 & 0
\end{array}\right) \quad \text { and } \quad \omega_{\mathfrak{e}}=\left(\begin{array}{cccccc}
1 & 1 & -1 & 0 & 0 & -1 \\
0 & -1 & 1 & -1 & 0 & 1 \\
0 & 0 & 0 & -1 & 0 & -1 \\
0 & 0 & 0 & 0 & -1 & -1 \\
0 & 1 & 0 & 0 & 0 & -1 \\
0 & -1 & 0 & 1 & -1 & 0
\end{array}\right) \text {, }
$$


given in the lattice basis as matrices from $\mathrm{GL}(6, \mathbb{Z})$, for example, $\vartheta_{\mathfrak{e}} e_{1}=e_{6}$. One can go to the $\mathrm{SO}(6)$ form by a basis change $\vartheta=B_{\mathfrak{e}} \vartheta_{\mathfrak{e}} B_{\mathfrak{e}}^{-1}$ and $\omega=B_{\mathfrak{e}} \omega_{\mathfrak{e}} B_{\mathfrak{e}}^{-1}$, where the columns of $B_{\mathfrak{e}}$ are the basis vectors $e_{i}, i=1, \ldots, 6$. In the $\mathrm{SU}(3)$ basis these generators read (see table C.2 of [32])

$$
\vartheta^{(3)}=\left(\begin{array}{lll}
0 & 1 & 0 \\
0 & 0 & 1 \\
1 & 0 & 0
\end{array}\right) \text { and } \omega^{(3)}=\left(\begin{array}{ccc}
1 & 0 & 0 \\
0 & \mathrm{e}^{2 \pi \mathrm{i} \frac{1}{3}} & 0 \\
0 & 0 & \mathrm{e}^{2 \pi \mathrm{i} \frac{2}{3}}
\end{array}\right),
$$

which generate an irreducible three-dimensional representation 3 of $\Delta(27)$.

The number of untwisted moduli corresponds to the number of invariant singlets within the tensor products of the three-dimensional representation and its conjugate:

$$
\begin{aligned}
& \mathbf{3} \otimes \overline{\mathbf{3}} \rightarrow \mathbf{1}_{\mathbf{0}} \oplus \mathbf{1}_{\mathbf{1}} \oplus \mathbf{1}_{\mathbf{2}} \oplus \mathbf{1}_{\mathbf{3}} \oplus \mathbf{1}_{\mathbf{4}} \oplus \mathbf{1}_{\mathbf{5}} \oplus \mathbf{1}_{\mathbf{6}} \oplus \mathbf{1}_{\mathbf{7}} \oplus \mathbf{1}_{\mathbf{8}} \\
& \mathbf{3} \otimes \mathbf{3} \rightarrow \overline{\mathbf{3}} \oplus \overline{\mathbf{3}} \oplus \overline{\mathbf{3}},
\end{aligned}
$$

where only $\mathbf{1}_{\mathbf{0}}$ in equation (3.28a) denotes a $\Delta(27)$-invariant singlet. Therefore, by using equation (2.7), we conclude that there is only one orbifold-invariant untwisted Kähler modulus and no orbifold-invariant untwisted complex-structure modulus, i.e.

$$
\left(h_{\mathrm{U}}^{(1,1)}, h_{\mathrm{U}}^{(2,1)}\right)=(1,0) .
$$

The only nonvanishing contributions to the Hodge numbers from the twisted sectors arise from the 27 fixed points of the $T_{\left[\vartheta \omega \vartheta^{2} \omega^{2}\right]}$ sector, which are inequivalent on the torus. The constructing elements associated to these fixed points are $g^{(i)}=\left(\vartheta \omega \vartheta^{2} \omega^{2}, \lambda^{(i)}\right)$ with:

$$
\begin{array}{lll}
\lambda^{(1)}=\frac{1}{3}\left(-2 e_{1}+5 e_{2}+e_{3}-e_{4}-e_{5}+5 e_{6}\right), & \lambda^{(2)}=\frac{1}{3}\left(-2 e_{1}+5 e_{2}+e_{3}+2 e_{4}-e_{5}+5 e_{6}\right), \\
\lambda^{(3)}=\frac{1}{3}\left(e_{1}+2 e_{2}+e_{3}-e_{4}-e_{5}+2 e_{6}\right), & \lambda^{(4)}=\frac{1}{3}\left(e_{1}+2 e_{2}+e_{3}+2 e_{4}-e_{5}+2 e_{6}\right), \\
\lambda^{(5)}=\frac{1}{3}\left(e_{1}+2 e_{2}+e_{3}+5 e_{4}-e_{5}+2 e_{6}\right), & \lambda^{(6)}=\frac{1}{3}\left(e_{1}+5 e_{2}+e_{3}-e_{4}-e_{5}+2 e_{6}\right), \\
\lambda^{(7)}=\frac{1}{3}\left(e_{1}+5 e_{2}+e_{3}+2 e_{4}-e_{5}+2 e_{6}\right), & \lambda^{(8)}=\frac{1}{3}\left(e_{1}+5 e_{2}+e_{3}+5 e_{4}-e_{5}+2 e_{6}\right), \\
\lambda^{(9)}=\frac{1}{3}\left(-2 e_{1}+5 e_{2}-2 e_{3}-e_{4}-4 e_{5}+5 e_{6}\right), & \lambda^{(10)}=\frac{1}{3}\left(-2 e_{1}+5 e_{2}-2 e_{3}-e_{4}-e_{5}+5 e_{6}\right), \\
\lambda^{(11)}=\frac{1}{3}\left(-2 e_{1}+5 e_{2}+e_{3}-e_{4}-4 e_{5}+5 e_{6}\right), & \lambda^{(12)}=\frac{1}{3}\left(-2 e_{1}+5 e_{2}+e_{3}+2 e_{4}-4 e_{5}+5 e_{6}\right), \\
\lambda^{(13)}=\frac{1}{3}\left(-2 e_{1}+8 e_{2}-2 e_{3}-e_{4}-4 e_{5}+5 e_{6}\right), & \lambda^{(14)}=\frac{1}{3}\left(-2 e_{1}+8 e_{2}+e_{3}-e_{4}-4 e_{5}+5 e_{6}\right), \\
\lambda^{(15)}=\frac{1}{3}\left(-2 e_{1}+8 e_{2}+e_{3}+2 e_{4}-4 e_{5}+5 e_{6}\right), & \lambda^{(16)}=\frac{1}{3}\left(e_{1}+2 e_{2}-2 e_{3}-e_{4}-4 e_{5}+5 e_{6}\right), \\
\lambda^{(17)}=\frac{1}{3}\left(e_{1}+2 e_{2}-2 e_{3}+2 e_{4}-4 e_{5}+5 e_{6}\right), & \lambda^{(18)}=\frac{1}{3}\left(e_{1}+2 e_{2}+e_{3}+2 e_{4}-4 e_{5}+5 e_{6}\right), \\
\lambda^{(19)}=\frac{1}{3}\left(e_{1}+5 e_{2}-2 e_{3}-e_{4}-4 e_{5}+5 e_{6}\right), & \lambda^{(20)}=\frac{1}{3}\left(e_{1}+5 e_{2}-2 e_{3}+2 e_{4}-4 e_{5}+5 e_{6}\right), \\
\lambda^{(21)}=\frac{1}{3}\left(e_{1}+5 e_{2}+e_{3}-e_{4}-4 e_{5}+2 e_{6}\right), & \lambda^{(22)}=\frac{1}{3}\left(e_{1}+5 e_{2}+e_{3}+2 e_{4}-4 e_{5}+2 e_{6}\right), \\
\lambda^{(23)}=\frac{1}{3}\left(e_{1}+5 e_{2}+e_{3}+2 e_{4}-4 e_{5}+5 e_{6}\right), & \lambda^{(24)}=\frac{1}{3}\left(e_{1}+5 e_{2}+e_{3}+5 e_{4}-4 e_{5}+2 e_{6}\right), \\
\lambda^{(25)}=\frac{1}{3}\left(e_{1}+8 e_{2}-2 e_{3}-e_{4}-4 e_{5}+5 e_{6}\right), & \lambda^{(26)}=\frac{1}{3}\left(e_{1}+8 e_{2}-2 e_{3}+2 e_{4}-4 e_{5}+5 e_{6}\right), \\
\lambda^{(27)}=\frac{1}{3}\left(e_{1}+8 e_{2}+e_{3}+2 e_{4}-4 e_{5}+5 e_{6}\right) . &
\end{array}
$$


Out of these 27 constructing elements, only three are inequivalent on the orbifold. We choose $g^{(1)}, g^{(2)}$ and $g^{(3)}$. The corresponding fixed points are localized at $f^{(i)}=\frac{1}{9} \hat{f}_{\alpha}^{(i)} e_{\alpha}$, $i=1,2,3$, with

$$
\hat{f}^{(1)}=(1,1,6,5,6,7), \hat{f}^{(2)}=(1,1,3,8,6,7), \hat{f}^{(3)}=(4,1,6,2,3,1) .
$$

Since these are fixed points (and not tori) and the centralizers of $g^{(i)}$ are trivial, the $T_{\left[\vartheta \omega \vartheta^{2} \omega^{2}\right]}$ twisted sector combines with the inverse twisted sector $T_{\left[\vartheta \omega^{2} \vartheta^{2} \omega\right]}$ yielding three left-chiral 27-plets plus their CPT conjugate partners. Hence, the only twisted contribution to the Hodge numbers is $(3,0)$.

In summary, the Hodge numbers are $\left(h^{(1,1)}, h^{(2,1)}\right)=(4,0)$ originating from the various sectors as

$$
(1,0) U+(3,0) T_{\left[\vartheta \omega \vartheta^{2} \omega^{2}\right]} .
$$

The main feature that distinguishes this case from the previous examples is the existence of a non-trivial fundamental group $\pi_{1}=S /\langle F\rangle$. The group $\langle F\rangle$ generated by the set $F$ of the constructing elements listed in equation (3.30) contains the full lattice $\Lambda$ of the space group $S$ and a (normal subgroup) $\mathbb{Z}_{3} \subset \Delta(27)$ generated by $\vartheta \omega \vartheta^{2} \omega^{2}$. Thus, we identify the fundamental group of the $\Delta(27)-1-4$ orbifold as

$$
\pi_{1}=S /\langle F\rangle=\Delta(27) / \mathbb{Z}_{3}=\mathbb{Z}_{3} \times \mathbb{Z}_{3} .
$$

\section{Summary and discussion}

We have computed systematically the number of (untwisted and twisted) moduli and fundamental groups of all 331 recently classified [32] $\mathcal{N}=1$ non-Abelian (symmetric) orbifold compactifications of the $\mathrm{E}_{8} \times \mathrm{E}_{8}^{\prime}$ heterotic string with standard gauge embedding. We have developed the tools that allow us to determine the number of Kähler and complexstructure moduli by using group-theoretical and geometrical properties of the orbifolds rather than by direct computation. Our results are presented in table 2, where the Hodge numbers, classified by sector, are displayed. Furthermore, we list all 38 non-trivial fundamental groups in table 3. Further details (such as orbifold generators, constructing elements, non-trivial centralizer elements, compactification lattices, etc.) are made available at http://einrichtungen.physik.tu-muenchen.de/T30e/codes/NonAbelianOrbifolds/ in a Mathematica-compatible format.

Most of the fundamental groups (35 out of 38) are Abelian (see table 3), such as $\mathbb{Z}_{2}$, $\mathbb{Z}_{3}, \mathbb{Z}_{4}$ and $\mathbb{Z}_{2} \times \mathbb{Z}_{2} .{ }^{6}$ In 14 cases the fundamental group is generated by translations, in 16 cases all generators are rotations and in the remaining 8 cases the fundamental group is generated by translations and rotations. From a phenomenological point of view, orbifolds with non-trivial fundamental groups are very interesting as they may allow for non-local GUT breaking, which can improve gauge coupling unification. Furthermore, it would be interesting to study the connection of these orbifolds to smooth Calabi-Yau spaces [11, 40],

\footnotetext{
${ }^{6}$ This is in contrast to smooth Calabi-Yau spaces, which have a much wider variety of fundamental groups, see e.g. [58, 59] for fundamental groups of complete intersection Calabi-Yau threefolds.
} 
since the standard model gauge group (especially the hypercharge) can survive a full blowup of the orbifold to a smooth Calabi-Yau when the fundamental group of the orbifold is non-trivial and the gauge group is broken non-locally.

Besides the fact that, like almost all Abelian cases, most non-Abelian orbifold geometries satisfy the relation $\chi=0 \bmod 12$, for which we have no explanation, we observe that, in contrast to Abelian orbifolds, there is a large number of geometries (and a greater number of models) with the overall volume modulus as the only untwisted modulus available. These models should be further analyzed in the context of no-scale supergravity. Note also that this might be a positive feature for moduli stabilization, although unfortunately it prevents anisotropic compactifications, which are desirable to solve the tension between the string scale and the GUT scale [36, 60].

An interesting observation is that 42 out of the 331 orbifold geometries have vanishing Euler numbers $\chi$ (i.e. $\left.h^{(1,1)}=h^{(2,1)}\right)$. In these cases we note that we have $h_{\mathrm{U}}^{(1,1)}=h_{\mathrm{U}}^{(2,1)}$ and $h_{\mathrm{T}}^{(1,1)}=h_{\mathrm{T}}^{(2,1)}$, independently. The latter, $h_{\mathrm{T}}^{(1,1)}=h_{\mathrm{T}}^{(2,1)}$, is related to higher-dimensional supersymmetry. Hence, 4D chiral spectra can never be obtained in these cases using standard heterotic orbifold CFT techniques alone. The inclusion of magnetized tori [54, 61] may offer a plausible way to circumvent this hurdle. However, their description is only known in blow-up, but not on the singular orbifold.

Furthermore, it would be interesting to analyze the cases of vanishing Euler numbers in the context of [62], which states that type II string theory compactified on CalabiYau threefolds with vanishing Euler numbers leads to $\mathcal{N}=4$ enhanced supersymmetry (spontaneously broken to $\mathcal{N}=2$ ). Translated to the case of heterotic orbifolds with standard embedding and vanishing Euler numbers, one might expect $\mathcal{N}=2$ enhanced supersymmetry (spontaneously broken to $\mathcal{N}=1$ ). In addition, we find cases where $h_{\mathrm{T}}^{(1,1)}=$ $h_{\mathrm{T}}^{(2,1)}=0$, for example, $D_{4}-1-6$ has only untwisted Hodge numbers $(2,2)$ (see table 2 ) and there are few other similar cases in Abelian orbifolds [32]. One might conjecture that these cases give even higher enhanced supersymmetry, i.e. (spontaneously broken) $\mathcal{N}=4$. On the other hand, there are cases of orbifolds with vanishing Euler numbers where the orbifold intuition naively contradicts the general results of [62]: e.g. consider the first case of table 2, $S_{3}-1-1$, with Hodge numbers $(15,15)$ decomposed as $(2,2) U+(4,4) T_{[\vartheta]}+(9,9) T_{[\omega]}$. In this case, the two twisted sectors $T_{[\vartheta]}$ and $T_{[\omega]}$ both feel $\mathcal{N}=2$ supersymmetry, while the untwisted sector $U$ is $\mathcal{N}=1$ in four dimensions. However, $T_{[\vartheta]}$ has different $\mathcal{N}=2$ than $T_{[\omega]}$, as one can easily verify by noticing that the generators $\vartheta$ and $\omega$ leave untouched different two-tori. This implies that the full action of the orbifold breaks explicitly (not spontaneously) $\mathcal{N}=2$ to $\mathcal{N}=1$ supersymmetry, even though $\chi=0$.

In addition, we have presented the details of three sample models with the point groups $S_{3}$ (confirming the results of ref. [34]), $T_{7}$ and $\Delta(27)$. We have chosen these point groups because they illustrate the main properties of non-Abelian orbifold compactifications and because of their relevance in particle physics, for example in the context of neutrino mixing and family symmetries (see e.g. [63-65]). As in the Abelian case, we expect the (nonAbelian) point group of the orbifold to be in close connection with the family symmetry of the $4 \mathrm{D}$ effective theory via string-selection rules [22]. If this was the case, our examples 
would be of phenomenological interest. Yet the specifics of the string selection rules for non-Abelian orbifolds should still be worked out.

The results of this work lay the foundation stone of future phenomenological studies based on non-Abelian orbifolds and can be extended in various ways. Particularly, it would be interesting to extend this study to type IIA strings on orientifolds [27, 66, 67], where appealing phenomenology can also emerge. Likewise, it might be desirable to apply our techniques to compactifications of the heterotic strings on four-dimensional orbifolds, in order to reveal further connections to K3 manifolds [68, 69]. Finally, one is now in position to tackle the technical details of the gauge embedding in order to possibly arrive at promising constructions. In this respect, it is phenomenologically relevant to emphasize that in general the rank of the gauge group shall be reduced for non-Abelian orbifolds, which is in contrast to the situation in Abelian orbifolds, where the rank is always 16 after compactification. This can help avoiding multiple Higgs mechanisms to arrive at phenomenologically viable constructions from string theory.

\section{Acknowledgments}

We would like to thank Sebastian Konopka, Jan Louis and Michael Ratz for useful discussions. The authors thank the Bethe Center for Theoretical Physics in Bonn, where part of this project was done, for hospitality and support. P.V. is supported by SFB grant 676 and is grateful to the Institute of Physics, UNAM, for hospitality and support. S.R-S. is partially supported by CONACyT grant 151234 and DGAPA-PAPIIT grant IB101012. M.F. is supported by the Deutsche Forschungsgemeinschaft (DFG) through the cluster of excellence "Origin and Structure of the Universe".

\section{A Results}

In this appendix we list the generators of the orbifolding group, the total Hodge numbers, their contributions from the various twisted and untwisted sectors and the mechanism of higher-dimensional gauge group breaking (local or non-local, see table 3) for all 331 orbifolds with non-Abelian point group. For example, consider the $S_{3}$ point group with $\mathbb{Z}$-class \# 6 and affine class \# 1 (i.e. no roto-translations and the orbifolding group is generated by $(\vartheta, 0)$ and $(\omega, 0))$. The higher-dimensional gauge group is broken locally in higher dimensions, which corresponds to a trivial fundamental group. The Hodge numbers are $(7,7)$, where $(2,2)$ originate from the untwisted sector $U,(4,4)$ from the twisted sector $T_{[\vartheta]}$ and, finally, $(1,1)$ from $T_{[\omega]}$. 


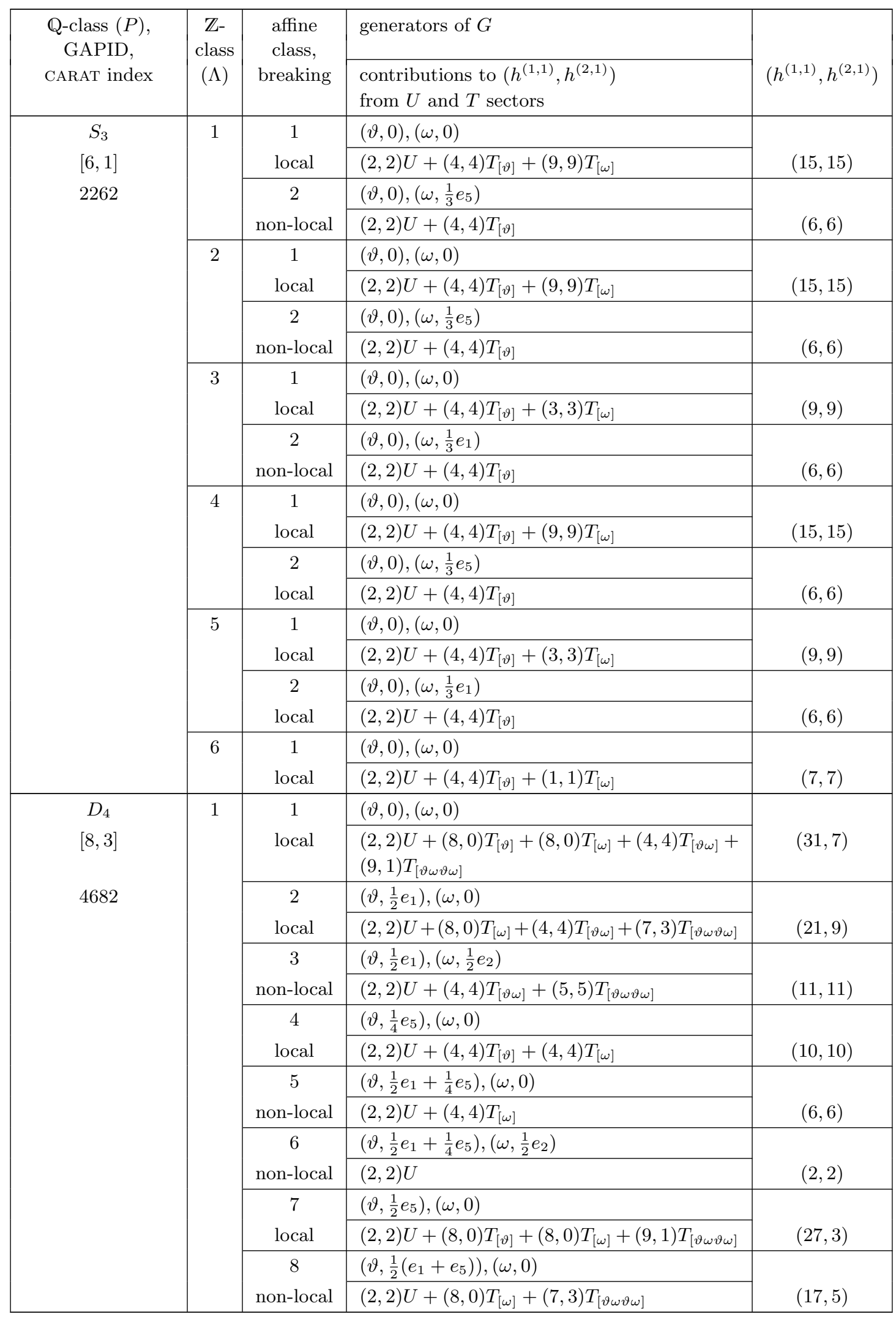

(Table 2. Continued...) 


\begin{tabular}{|c|c|c|c|c|}
\hline \multirow{2}{*}{$\begin{array}{l}\text { Q-class }(P), \\
\text { GAPID, } \\
\text { CARAT index }\end{array}$} & \multirow{2}{*}{$\begin{array}{c}\mathbb{Z}- \\
\text { class } \\
(\Lambda)\end{array}$} & \multirow{2}{*}{$\begin{array}{c}\text { affine } \\
\text { class, } \\
\text { breaking }\end{array}$} & generators of $G$ & \multirow[b]{2}{*}{$\left(h^{(1,1)}, h^{(2,1)}\right)$} \\
\hline & & & $\begin{array}{l}\text { contributions to }\left(h^{(1,1)}, h^{(2,1)}\right) \\
\text { from } U \text { and } T \text { sectors }\end{array}$ & \\
\hline & & \multirow{2}{*}{$\begin{array}{c}9 \\
\text { non-local }\end{array}$} & $\left(\vartheta, \frac{1}{2}\left(e_{1}+e_{5}\right)\right),\left(\omega, \frac{1}{2} e_{2}\right)$ & \multirow[b]{2}{*}{$(7,7)$} \\
\hline & & & $(2,2) U+(5,5) T_{[\vartheta \omega \vartheta \omega]}$ & \\
\hline & \multirow[t]{16}{*}{2} & \multirow{2}{*}{$\begin{array}{c}1 \\
\text { local }\end{array}$} & $(\vartheta, 0),(\omega, 0)$ & \multirow[b]{2}{*}{$(22,4)$} \\
\hline & & & $\begin{array}{l}(2,2) U+(4,0) T_{[\vartheta]}+(8,0) T_{[\omega]}+(2,2) T_{[\vartheta \omega]}+ \\
(6,0) T_{[\vartheta \omega \vartheta \omega]}\end{array}$ & \\
\hline & & \multirow{2}{*}{$\begin{array}{c}2 \\
\text { local }\end{array}$} & $\left(\vartheta, \frac{1}{2} e_{5}\right),(\omega, 0)$ & \multirow[b]{2}{*}{$(13,7)$} \\
\hline & & & $(2,2) U+(4,0) T_{[\vartheta]}+(4,4) T_{[\omega]}+(3,1) T_{[\vartheta \omega \vartheta \omega]}$ & \\
\hline & & \multirow{2}{*}{$\begin{array}{c}3 \\
\text { local }\end{array}$} & $(\vartheta, 0),\left(\omega, \frac{1}{2} e_{1}\right)$ & \multirow[b]{2}{*}{$(12,6)$} \\
\hline & & & $(2,2) U+(4,0) T_{[\vartheta]}+(2,2) T_{[\vartheta \omega]}+(4,2) T_{[\vartheta \omega \vartheta \omega]}$ & \\
\hline & & \multirow{2}{*}{$\begin{array}{c}4 \\
\text { non-local }\end{array}$} & $\left(\vartheta, \frac{1}{2} e_{5}\right),\left(\omega, \frac{1}{2} e_{1}\right)$ & \multirow[b]{2}{*}{$(9,3)$} \\
\hline & & & $(2,2) U+(4,0) T_{[\vartheta]}+(3,1) T_{[\vartheta \omega \vartheta \omega]}$ & \\
\hline & & \multirow{2}{*}{$\begin{array}{c}5 \\
\text { local }\end{array}$} & $\left(\vartheta, \frac{1}{4} e_{3}\right),(\omega, 0)$ & \multirow[b]{2}{*}{$(8,8)$} \\
\hline & & & $(2,2) U+(2,2) T_{[\vartheta]}+(4,4) T_{[\omega]}$ & \\
\hline & & \multirow{2}{*}{$\begin{array}{c}6 \\
\text { non-local }\end{array}$} & $\left(\vartheta, \frac{1}{4} e_{3}\right),\left(\omega, \frac{1}{2} e_{1}\right)$ & \multirow[b]{2}{*}{$(4,4)$} \\
\hline & & & $(2,2) U+(2,2) T_{[\vartheta]}$ & \\
\hline & & \multirow{2}{*}{$\begin{array}{c}7 \\
\text { local }\end{array}$} & $\left(\vartheta, \frac{1}{2} e_{3}\right),(\omega, 0)$ & \multirow[b]{2}{*}{$(20,2)$} \\
\hline & & & $(2,2) U+(4,0) T_{[\vartheta]}+(8,0) T_{[\omega]}+(6,0) T_{[\vartheta \omega \vartheta \omega]}$ & \\
\hline & & \multirow{2}{*}{$\begin{array}{c}8 \\
\text { non-local }\end{array}$} & $\left(\vartheta, \frac{1}{2} e_{3}\right),\left(\omega, \frac{1}{2} e_{1}\right)$ & \multirow[b]{2}{*}{$(10,4)$} \\
\hline & & & $(2,2) U+(4,0) T_{[\vartheta]}+(4,2) T_{[\vartheta \omega \vartheta \omega]}$ & \\
\hline & \multirow[t]{8}{*}{3} & \multirow{2}{*}{$\begin{array}{c}1 \\
\text { local }\end{array}$} & $(\vartheta, 0),(\omega, 0)$ & \multirow[b]{2}{*}{$(17,5)$} \\
\hline & & & $\begin{array}{l}(2,2) U+(4,0) T_{[\vartheta]}+(4,0) T_{[\omega]}+(2,2) T_{[\vartheta \omega]}+ \\
(5,1) T_{[\vartheta \omega \vartheta \omega]}\end{array}$ & \\
\hline & & \multirow{2}{*}{$\begin{array}{c}2 \\
\text { local }\end{array}$} & $\left(\vartheta, \frac{1}{2} e_{3}\right),(\omega, 0)$ & \multirow[b]{2}{*}{$(14,2)$} \\
\hline & & & $(2,2) U+(4,0) T_{[\vartheta]}+(4,0) T_{[\omega]}+(4,0) T_{[\vartheta \omega \vartheta \omega]}$ & \\
\hline & & 3 & $\left(\vartheta, \frac{1}{4} e_{1}\right),(\omega, 0)$ & \\
\hline & & local & $(2,2) U+(2,2) T_{[\vartheta]}+(2,2) T_{[\omega]}$ & $(6,6)$ \\
\hline & & 4 & $\left(\vartheta, \frac{1}{2} e_{1}\right),(\omega, 0)$ & \\
\hline & & local & $(2,2) U+(4,0) T_{[\vartheta]}+(4,0) T_{[\omega]}+(5,1) T_{[\vartheta \omega \vartheta \omega]}$ & $(15,3)$ \\
\hline & 4 & 1 & $(\vartheta, 0),(\omega, 0)$ & \\
\hline & & local & $\begin{array}{l}(2,2) U+(4,0) T_{[\vartheta]}+(4,0) T_{[\omega]}+(1,1) T_{[\vartheta \omega]}+ \\
(4,0) T_{[\vartheta \omega \vartheta \omega]}\end{array}$ & $(15,3)$ \\
\hline & & 2 & $\left(\vartheta, \frac{1}{2} e_{3}\right),(\omega, 0)$ & \\
\hline & & local & $(2,2) U+(4,0) T_{[\vartheta]}+(2,2) T_{[\omega]}+(2,0) T_{[\vartheta \omega \vartheta \omega]}$ & $(10,4)$ \\
\hline & & 3 & $\left(\vartheta, \frac{1}{2} e_{3}\right),\left(\omega, \frac{1}{2} e_{4}\right)$ & \\
\hline & & local & $(2,2) U+(2,2) T_{[\vartheta]}+(2,2) T_{[\omega]}+(1,1) T_{[\vartheta \omega \vartheta \omega]}$ & $(7,7)$ \\
\hline & 5 & 1 & $(\vartheta, 0),(\omega, 0)$ & \\
\hline & & local & $\begin{array}{l}(2,2) U+(4,0) T_{[\vartheta]}+(16,0) T_{[\omega]}+(4,4) T_{[\vartheta \omega]}+ \\
(10,0) T_{[\vartheta \omega \vartheta \omega]}\end{array}$ & $(36,6)$ \\
\hline & & 2 & $(\vartheta, 0),\left(\omega, \frac{1}{2} e_{1}\right)$ & \\
\hline & & local & $(2,2) U+(4,0) T_{[\vartheta]}+(4,4) T_{[\vartheta \omega]}+(6,4) T_{[\vartheta \omega \vartheta \omega]}$ & $(16,10)$ \\
\hline
\end{tabular}

(Table 2. Continued ...) 


\begin{tabular}{|c|c|c|c|c|}
\hline \multirow{2}{*}{$\begin{array}{l}\text { Q-class }(P), \\
\text { GAPID, } \\
\text { CARAT index }\end{array}$} & \multirow{2}{*}{$\begin{array}{c}\mathbb{Z}^{-} \\
\text {class } \\
(\Lambda)\end{array}$} & \multirow{2}{*}{$\begin{array}{c}\text { affine } \\
\text { class, } \\
\text { breaking }\end{array}$} & generators of $G$ & \multirow[b]{2}{*}{$\left(h^{(1,1)}, h^{(2,1)}\right)$} \\
\hline & & & $\begin{array}{l}\text { contributions to }\left(h^{(1,1)}, h^{(2,1)}\right) \\
\text { from } U \text { and } T \text { sectors }\end{array}$ & \\
\hline & & 3 & $\left(\vartheta, \frac{1}{4} e_{5}\right),(\omega, 0)$ & \multirow[b]{2}{*}{$(12,12)$} \\
\hline & & local & $(2,2) U+(2,2) T_{[\vartheta]}+(8,8) T_{[\omega]}$ & \\
\hline & & \multirow{2}{*}{$\begin{array}{c}4 \\
\text { non-local }\end{array}$} & $\left(\vartheta, \frac{1}{4} e_{5}\right),\left(\omega, \frac{1}{2} e_{1}\right)$ & \multirow[b]{2}{*}{$(4,4)$} \\
\hline & & & $(2,2) U+(2,2) T_{[\vartheta]}$ & \\
\hline & & \multirow{2}{*}{$\begin{array}{c}5 \\
\text { local }\end{array}$} & $\left(\vartheta, \frac{1}{2} e_{5}\right),(\omega, 0)$ & \multirow[b]{2}{*}{$(32,2)$} \\
\hline & & & $(2,2) U+(4,0) T_{[\vartheta]}+(16,0) T_{[\omega]}+(10,0) T_{[\vartheta \omega \vartheta \omega]}$ & \\
\hline & & \multirow{2}{*}{$\begin{array}{c}6 \\
\text { non-local }\end{array}$} & $\left(\vartheta, \frac{1}{2} e_{5}\right),\left(\omega, \frac{1}{2} e_{1}\right)$ & \multirow[b]{2}{*}{$(12,6)$} \\
\hline & & & $(2,2) U+(4,0) T_{[\vartheta]}+(6,4) T_{[\vartheta \omega \vartheta \omega]}$ & \\
\hline & \multirow[t]{16}{*}{6} & \multirow{2}{*}{$\begin{array}{c}1 \\
\text { local }\end{array}$} & $(\vartheta, 0),(\omega, 0)$ & \multirow[b]{2}{*}{$(22,4)$} \\
\hline & & & $\begin{array}{l}(2,2) U+(4,0) T_{[\vartheta]}+(8,0) T_{[\omega]}+(2,2) T_{[\vartheta \omega]}+ \\
(6,0) T_{[\vartheta \omega \vartheta \omega]}\end{array}$ & \\
\hline & & \multirow{2}{*}{$\begin{array}{c}2 \\
\text { local }\end{array}$} & $(\vartheta, 0),\left(\omega, \frac{1}{2} e_{4}\right)$ & \multirow[b]{2}{*}{$(16,4)$} \\
\hline & & & $(2,2) U+(2,2) T_{[\vartheta]}+(8,0) T_{[\omega]}+(4,0) T_{[\vartheta \omega \vartheta \omega]}$ & \\
\hline & & \multirow{2}{*}{$\begin{array}{c}3 \\
\text { non-local }\end{array}$} & $(\vartheta, 0),\left(\omega, \frac{1}{2} e_{1}\right)$ & \multirow[b]{2}{*}{$(12,6)$} \\
\hline & & & $(2,2) U+(4,0) T_{[\vartheta]}+(2,2) T_{[\vartheta \omega]}+(4,2) T_{[\vartheta \omega \vartheta \omega]}$ & \\
\hline & & \multirow{2}{*}{$\begin{array}{c}4 \\
\text { non-local } \\
\end{array}$} & $(\vartheta, 0),\left(\omega, \frac{1}{2}\left(e_{1}+e_{4}\right)\right)$ & \multirow[b]{2}{*}{$(6,6)$} \\
\hline & & & $(2,2) U+(2,2) T_{[\vartheta]}+(2,2) T_{[\vartheta \omega \vartheta \omega]}$ & \\
\hline & & \multirow{2}{*}{$\begin{array}{c}5 \\
\text { local }\end{array}$} & $\left(\vartheta, \frac{1}{4} e_{3}\right),(\omega, 0)$ & \multirow[b]{2}{*}{$(8,8)$} \\
\hline & & & $(2,2) U+(2,2) T_{[\vartheta]}+(4,4) T_{[\omega]}$ & \\
\hline & & \multirow{2}{*}{$\begin{array}{c}6 \\
\text { non-local } \\
\end{array}$} & $\left(\vartheta, \frac{1}{4} e_{3}\right),\left(\omega, \frac{1}{2} e_{1}\right)$ & \multirow[b]{2}{*}{$(4,4)$} \\
\hline & & & $(2,2) U+(2,2) T_{[\vartheta]}$ & \\
\hline & & 7 & $\left(\vartheta, \frac{1}{2} e_{3}\right),(\omega, 0)$ & \multirow[b]{2}{*}{$(20,2)$} \\
\hline & & local & $(2,2) U+(4,0) T_{[\vartheta]}+(8,0) T_{[\omega]}+(6,0) T_{[\vartheta \omega \vartheta \omega]}$ & \\
\hline & & \multirow{2}{*}{$\begin{array}{c}8 \\
\text { non-local }\end{array}$} & $\left(\vartheta, \frac{1}{2} e_{3}\right),\left(\omega, \frac{1}{2} e_{1}\right)$ & \multirow[b]{2}{*}{$(10,4)$} \\
\hline & & & $(2,2) U+(4,0) T_{[\vartheta]}+(4,2) T_{[\vartheta \omega \vartheta \omega]}$ & \\
\hline & \multirow[t]{4}{*}{7} & 1 & $(\vartheta, 0),(\omega, 0)$ & \\
\hline & & local & $\begin{array}{l}(2,2) U+(4,0) T_{[\vartheta]}+(4,0) T_{[\omega]}+(1,1) T_{[\vartheta \omega]}+ \\
(4,0) T_{[\vartheta \omega \vartheta \omega]}\end{array}$ & $(15,3)$ \\
\hline & & 2 & $(\vartheta, 0),\left(\omega, \frac{1}{2} e_{4}\right)$ & \\
\hline & & local & $(2,2) U+(2,2) T_{[\vartheta]}+(4,0) T_{[\omega]}+(2,0) T_{[\vartheta \omega \vartheta \omega]}$ & $(10,4)$ \\
\hline & 8 & 1 & $(\vartheta, 0),(\omega, 0)$ & \\
\hline & & local & $\begin{array}{l}(2,2) U+(4,0) T_{[\vartheta]}+(4,0) T_{[\omega]}+(4,4) T_{[\vartheta \omega]}+ \\
(7,3) T_{[\vartheta \omega \vartheta \omega]}\end{array}$ & $(21,9)$ \\
\hline & & 2 & $\left(\vartheta, \frac{1}{4} e_{5}\right),(\omega, 0)$ & \\
\hline & & non-local & $(2,2) U+(2,2) T_{[\vartheta]}+(2,2) T_{[\omega]}$ & $(6,6)$ \\
\hline & & 3 & $\left(\vartheta, \frac{1}{2} e_{5}\right),(\omega, 0)$ & \\
\hline & & local & $(2,2) U+(4,0) T_{[\vartheta]}+(4,0) T_{[\omega]}+(7,3) T_{[\vartheta \omega \vartheta \omega]}$ & $(17,5)$ \\
\hline & 9 & 1 & $(\vartheta, 0),(\omega, 0)$ & \\
\hline & & non-local & $\begin{array}{l}(2,2) U+(4,0) T_{[\vartheta]}+(4,0) T_{[\omega]}+(2,2) T_{[\vartheta \omega]}+ \\
(5,1) T_{[\vartheta \omega \vartheta \omega]}\end{array}$ & $(17,5)$ \\
\hline
\end{tabular}

(Table 2. Continued ...) 


\begin{tabular}{|c|c|c|c|c|}
\hline \multirow{10}{*}{$\begin{array}{c}\text { Q-class }(P), \\
\text { GAPID, } \\
\text { CARAT index }\end{array}$} & \multirow{2}{*}{$\begin{array}{c}\mathbb{Z}- \\
\text { class } \\
(\Lambda)\end{array}$} & \multirow{2}{*}{$\begin{array}{c}\text { affine } \\
\text { class, } \\
\text { breaking }\end{array}$} & generators of $G$ & \multirow[b]{2}{*}{$\left(h^{(1,1)}, h^{(2,1)}\right)$} \\
\hline & & & $\begin{array}{l}\text { contributions to }\left(h^{(1,1)}, h^{(2,1)}\right) \\
\text { from } U \text { and } T \text { sectors }\end{array}$ & \\
\hline & & \multirow{2}{*}{$\begin{array}{c}2 \\
\text { non-local }\end{array}$} & $\left(\vartheta, \frac{1}{4} e_{1}\right),(\omega, 0)$ & \multirow[b]{2}{*}{$(6,6)$} \\
\hline & & & $(2,2) U+(2,2) T_{[\vartheta]}+(2,2) T_{[\omega]}$ & \\
\hline & & \multirow{2}{*}{$\begin{array}{c}3 \\
\text { non-local }\end{array}$} & $\left(\vartheta, \frac{1}{2} e_{1}\right),(\omega, 0)$ & \multirow[b]{2}{*}{$(15,3)$} \\
\hline & & & $(2,2) U+(4,0) T_{[\vartheta]}+(4,0) T_{[\omega]}+(5,1) T_{[\vartheta \omega \vartheta \omega]}$ & \\
\hline & & \multirow{2}{*}{$\begin{array}{c}4 \\
\text { local }\end{array}$} & $(\vartheta, 0),\left(\omega, \frac{1}{2}\left(e_{2}+\frac{1}{2} e_{3}+e_{5}\right)\right)$ & \multirow[b]{2}{*}{$(8,8)$} \\
\hline & & & $(2,2) U+(2,2) T_{[\vartheta]}+(2,2) T_{[\omega]}+(2,2) T_{[\vartheta \omega \vartheta \omega]}$ & \\
\hline & & \multirow{2}{*}{$\begin{array}{c}5 \\
\text { local }\end{array}$} & $(\vartheta, 0),\left(\omega, \frac{1}{2} e_{3}\right)$ & \multirow[b]{2}{*}{$(17,5)$} \\
\hline & & & $\begin{array}{l}(2,2) U+(4,0) T_{[\vartheta]}+(4,0) T_{[\omega]}+(2,2) T_{[\vartheta \omega]}+ \\
(5,1) T_{[\vartheta \omega \vartheta \omega]}\end{array}$ & \\
\hline$A_{4}$ & 1 & 1 & $(\vartheta, 0),(\omega, 0)$ & \multirow[b]{2}{*}{$(6,4)$} \\
\hline$[12,3]$ & & local & $(1,1) U+(1,1) T_{[\vartheta]}+(3,1) T_{[\omega]}+(1,1) T_{\left[\vartheta^{2}\right]}$ & \\
\hline \multirow[t]{28}{*}{4893} & \multirow[t]{6}{*}{2} & \multirow{2}{*}{$\begin{array}{c}1 \\
\text { non-local }\end{array}$} & $(\vartheta, 0),(\omega, 0)$ & \multirow[b]{2}{*}{$(11,3)$} \\
\hline & & & $(1,1) U+(1,1) T_{[\vartheta]}+(8,0) T_{[\omega]}+(1,1) T_{\left[\vartheta^{2}\right]}$ & \\
\hline & & \multirow{2}{*}{$\begin{array}{c}2 \\
\text { non-local }\end{array}$} & $(\vartheta, 0),\left(\omega, \frac{1}{2}\left(e_{1}+e_{2}\right)\right)$ & \multirow[b]{2}{*}{$(3,3)$} \\
\hline & & & $(1,1) U+(1,1) T_{[\vartheta]}+(1,1) T_{\left[\vartheta^{2}\right]}$ & \\
\hline & & \multirow{2}{*}{$\begin{array}{c}3 \\
\text { local }\end{array}$} & $(\vartheta, 0),\left(\omega, \frac{1}{2}\left(e_{1}+e_{3}\right)\right)$ & \multirow[b]{2}{*}{$(7,7)$} \\
\hline & & & $(1,1) U+(1,1) T_{[\vartheta]}+(4,4) T_{[\omega]}+(1,1) T_{\left[\vartheta^{2}\right]}$ & \\
\hline & \multirow[t]{2}{*}{3} & 1 & $(\vartheta, 0),(\omega, 0)$ & \multirow[b]{2}{*}{$(7,3)$} \\
\hline & & local & $(1,1) U+(1,1) T_{[\vartheta]}+(4,0) T_{[\omega]}+(1,1) T_{\left[\vartheta^{2}\right]}$ & \\
\hline & \multirow[t]{4}{*}{4} & 1 & $(\vartheta, 0),(\omega, 0)$ & \multirow[b]{2}{*}{$(7,3)$} \\
\hline & & non-local & $(1,1) U+(1,1) T_{[\vartheta]}+(4,0) T_{[\omega]}+(1,1) T_{\left[\vartheta^{2}\right]}$ & \\
\hline & & \multirow{2}{*}{$\begin{array}{c}2 \\
\text { non-local }\end{array}$} & $(\vartheta, 0),\left(\omega, \frac{1}{2}\left(e_{1}+e_{3}\right)\right)$ & \multirow[b]{2}{*}{$(5,5)$} \\
\hline & & & $(1,1) U+(1,1) T_{[\vartheta]}+(2,2) T_{[\omega]}+(1,1) T_{\left[\vartheta^{2}\right]}$ & \\
\hline & 5 & 1 & $(\vartheta, 0),(\omega, 0)$ & \\
\hline & & non-local & $(1,1) U+(1,1) T_{[\vartheta]}+(4,0) T_{[\omega]}+(1,1) T_{\left[\vartheta^{2}\right]}$ & $(7,3)$ \\
\hline & & 2 & $\left(\vartheta, \frac{1}{2}\left(e_{1}+e_{5}\right)\right),\left(\omega, \frac{1}{2}\left(e_{2}+e_{5}\right)\right)$ & \\
\hline & & local & $(1,1) U+(1,1) T_{[\vartheta]}+(2,2) T_{[\omega]}+(1,1) T_{\left[\vartheta^{2}\right]}$ & $(5,5)$ \\
\hline & 6 & 1 & $(\vartheta, 0),(\omega, 0)$ & \\
\hline & & local & $(1,1) U+(1,1) T_{[\vartheta]}+(16,0) T_{[\omega]}+(1,1) T_{\left[\vartheta^{2}\right]}$ & $(19,3)$ \\
\hline & & 2 & $(\vartheta, 0),\left(\omega, \frac{1}{2}\left(e_{2}+e_{4}\right)\right)$ & \\
\hline & & non-local & $(1,1) U+(1,1) T_{[\vartheta]}+(1,1) T_{\left[\vartheta^{2}\right]}$ & $(3,3)$ \\
\hline & 7 & 1 & $(\vartheta, 0),(\omega, 0)$ & \\
\hline & & local & $(1,1) U+(1,1) T_{[\vartheta]}+(8,0) T_{[\omega]}+(1,1) T_{\left[\vartheta^{2}\right]}$ & $(11,3)$ \\
\hline & & 2 & $\left(\vartheta, \frac{1}{2}\left(e_{1}+e_{3}+e_{5}\right)\right),\left(\omega, \frac{1}{2}\left(e_{1}+e_{2}\right)\right)$ & \\
\hline & & local & $(1,1) U+(1,1) T_{[\vartheta]}+(1,1) T_{\left[\vartheta^{2}\right]}$ & $(3,3)$ \\
\hline & 8 & 1 & $(\vartheta, 0),(\omega, 0)$ & \\
\hline & & local & $(1,1) U+(1,1) T_{[\vartheta]}+(4,0) T_{[\omega]}+(1,1) T_{\left[\vartheta^{2}\right]}$ & $(7,3)$ \\
\hline & 9 & 1 & $(\vartheta, 0),(\omega, 0)$ & \\
\hline & & local & $(1,1) U+(1,1) T_{[\vartheta]}+(4,0) T_{[\omega]}+(1,1) T_{\left[\vartheta^{2}\right]}$ & $(7,3)$ \\
\hline
\end{tabular}

(Table 2. Continued...) 


\begin{tabular}{|c|c|c|c|c|}
\hline \multirow{2}{*}{$\begin{array}{l}\text { Q-class }(P), \\
\text { GAPID, } \\
\text { CARAT index }\end{array}$} & \multirow{2}{*}{$\begin{array}{c}\mathbb{Z}- \\
\text { class } \\
(\Lambda)\end{array}$} & \multirow{2}{*}{$\begin{array}{c}\text { affine } \\
\text { class, } \\
\text { breaking }\end{array}$} & generators of $G$ & \multirow[b]{2}{*}{$\left(h^{(1,1)}, h^{(2,1)}\right)$} \\
\hline & & & $\begin{array}{l}\text { contributions to }\left(h^{(1,1)}, h^{(2,1)}\right) \\
\text { from } U \text { and } T \text { sectors }\end{array}$ & \\
\hline \multirow{16}{*}{$\begin{array}{c}D_{6} \\
{[12,4]}\end{array}$} & \multirow[t]{8}{*}{1} & \multirow{2}{*}{$\begin{array}{c}1 \\
\text { local }\end{array}$} & $(\vartheta, 0),(\omega, 0)$ & \multirow[b]{2}{*}{$(21,9)$} \\
\hline & & & $\begin{array}{l}(2,2) U+(4,0) T_{[\vartheta]}+(1,1) T_{[\omega]}+(4,0) T_{[\vartheta \omega]}+ \\
(5,5) T_{\left[\omega^{2}\right]}+(5,1) T_{\left[\omega^{3}\right]}\end{array}$ & \\
\hline & & \multirow{2}{*}{$\begin{array}{c}2 \\
\text { local } \\
\end{array}$} & $(\vartheta, 0),\left(\omega, \frac{1}{6} e_{5}\right)$ & \multirow[b]{2}{*}{$(6,6)$} \\
\hline & & & $(2,2) U+(2,2) T_{[\vartheta]}+(2,2) T_{[\vartheta \omega]}$ & \\
\hline & & \multirow{2}{*}{$\begin{array}{c}3 \\
\text { local }\end{array}$} & $(\vartheta, 0),\left(\omega, \frac{1}{3} e_{5}\right)$ & \multirow[b]{2}{*}{$(15,3)$} \\
\hline & & & $(2,2) U+(4,0) T_{[\vartheta]}+(4,0) T_{[\vartheta \omega]}+(5,1) T_{\left[\omega^{3}\right]}$ & \\
\hline & & \multirow{2}{*}{$\begin{array}{c}4 \\
\text { local }\end{array}$} & $(\vartheta, 0),\left(\omega, \frac{1}{2} e_{5}\right)$ & \multirow[b]{2}{*}{$(11,11)$} \\
\hline & & & $(2,2) U+(2,2) T_{[\vartheta]}+(2,2) T_{[\vartheta \omega]}+(5,5) T_{\left[\omega^{2}\right]}$ & \\
\hline & \multirow[t]{8}{*}{2} & \multirow{2}{*}{$\begin{array}{c}1 \\
\text { local }\end{array}$} & $(\vartheta, 0),(\omega, 0)$ & \multirow[b]{2}{*}{$(21,9)$} \\
\hline & & & $\begin{array}{l}(2,2) U+(4,0) T_{[\vartheta]}+(1,1) T_{[\omega]}+(4,0) T_{[\vartheta \omega]}+ \\
(5,5) T_{\left[\omega^{2}\right]}+(5,1) T_{\left[\omega^{3}\right]}\end{array}$ & \\
\hline & & \multirow{2}{*}{$\begin{array}{c}2 \\
\text { local }\end{array}$} & $(\vartheta, 0),\left(\omega, \frac{1}{6} e_{5}\right)$ & \multirow[b]{2}{*}{$(6,6)$} \\
\hline & & & $(2,2) U+(2,2) T_{[\vartheta]}+(2,2) T_{[\vartheta \omega]}$ & \\
\hline & & \multirow{2}{*}{$\begin{array}{c}3 \\
\text { local }\end{array}$} & $(\vartheta, 0),\left(\omega, \frac{1}{3} e_{5}\right)$ & \multirow[b]{2}{*}{$(15,3)$} \\
\hline & & & $(2,2) U+(4,0) T_{[\vartheta]}+(4,0) T_{[\vartheta \omega]}+(5,1) T_{\left[\omega^{3}\right]}$ & \\
\hline & & \multirow{2}{*}{$\begin{array}{c}4 \\
\text { local }\end{array}$} & $(\vartheta, 0),\left(\omega, \frac{1}{2} e_{5}\right)$ & \multirow[b]{2}{*}{$(11,11)$} \\
\hline & & & $(2,2) U+(2,2) T_{[\vartheta]}+(2,2) T_{[\vartheta \omega]}+(5,5) T_{\left[\omega^{2}\right]}$ & \\
\hline \multirow{14}{*}{$\begin{array}{c}\mathbb{Z}_{8} \rtimes \mathbb{Z}_{2} \\
{[16,6]}\end{array}$} & \multirow[t]{8}{*}{1} & \multirow{2}{*}{$\begin{array}{c}1 \\
\text { local }\end{array}$} & $(\vartheta, 0),(\omega, 0)$ & \multirow[b]{2}{*}{$(37,1)$} \\
\hline & & & $\begin{array}{l}(2,0) U+(4,0) T_{[\vartheta]}+(8,0) T_{[\omega]}+(10,0) T_{\left[\vartheta^{2}\right]}+ \\
(4,0) T_{[\vartheta \omega]}+(4,0) T_{\left[\vartheta^{2} \omega\right]}+(5,1) T_{\left[\vartheta^{4}\right]}\end{array}$ & \\
\hline & & \multirow{2}{*}{$\begin{array}{c}2 \\
\text { local }\end{array}$} & $\left(\vartheta, \frac{1}{2}\left(e_{2}+e_{4}\right)\right),\left(\omega, \frac{1}{2}\left(e_{1}+e_{2}+e_{3}+e_{4}\right)\right)$ & \multirow[b]{2}{*}{$(27,3)$} \\
\hline & & & $\begin{array}{l}(2,0) U+(4,0) T_{[\vartheta]}+(4,0) T_{[\omega]}+(6,0) T_{\left[\vartheta^{2}\right]}+ \\
(4,0) T_{[\vartheta \omega]}+(2,2) T_{\left[\vartheta^{2} \omega\right]}+(5,1) T_{\left[\vartheta^{4}\right]}\end{array}$ & \\
\hline & & \multirow{2}{*}{$\begin{array}{c}3 \\
\text { local }\end{array}$} & $(\vartheta, 0),\left(\omega, \frac{1}{2}\left(e_{5}+e_{6}\right)\right)$ & \multirow[b]{2}{*}{$(25,1)$} \\
\hline & & & $\begin{array}{l}(2,0) U+(4,0) T_{[\vartheta]}+(4,0) T_{[\omega]}+(6,0) T_{\left[\vartheta^{2}\right]}+ \\
(4,0) T_{[\vartheta \omega]}+(5,1) T_{\left[\vartheta^{4}\right]}\end{array}$ & \\
\hline & & 4 & $\left(\vartheta, \frac{1}{2}\left(e_{2}+e_{4}\right)\right),\left(\omega, \frac{1}{2}\left(e_{1}+e_{2}+e_{3}+e_{4}+e_{5}+e_{6}\right)\right)$ & \\
\hline & & local & $\begin{array}{l}(2,0) U+(4,0) T_{[\vartheta]}+(4,0) T_{[\omega]}+(6,0) T_{\left[\vartheta^{2}\right]}+ \\
(4,0) T_{[\vartheta \omega]}+(5,1) T_{\left[\vartheta^{4}\right]}\end{array}$ & $(25,1)$ \\
\hline & 2 & 1 & $(\vartheta, 0),(\omega, 0)$ & \\
\hline & & local & $\begin{array}{l}(2,0) U+(4,0) T_{[\vartheta]}+(6,0) T_{[\omega]}+(8,0) T_{\left[\vartheta^{2}\right]}+ \\
(4,0) T_{[\vartheta \omega]}+(2,0) T_{\left[\vartheta^{2} \omega\right]}+(4,0) T_{\left[\vartheta^{4}\right]}\end{array}$ & $(30,0)$ \\
\hline & & 2 & $\left(\vartheta, \frac{1}{2}\left(e_{2}+e_{4}+e_{5}\right)\right),\left(\omega, \frac{1}{2}\left(e_{2}+e_{3}+e_{4}+e_{6}\right)\right)$ & \\
\hline & & local & $\begin{array}{l}(2,0) U+(4,0) T_{[\vartheta]}+(2,2) T_{[\omega]}+(6,0) T_{\left[\vartheta^{2}\right]}+ \\
(4,0) T_{[\vartheta \omega]}+(3,1) T_{\left[\vartheta^{4}\right]}\end{array}$ & $(21,3)$ \\
\hline & & 3 & $(\vartheta, 0),\left(\omega, \frac{1}{2}\left(e_{1}+e_{3}\right)\right)$ & \\
\hline & & local & $\begin{array}{l}(2,0) U+(4,0) T_{[\vartheta]}+(6,0) T_{[\omega]}+(8,0) T_{\left[\vartheta^{2}\right]}+ \\
(4,0) T_{[\vartheta \omega]}+(2,0) T_{\left[\vartheta^{2} \omega\right]}+(4,0) T_{\left[\vartheta^{4}\right]}\end{array}$ & $(30,0)$ \\
\hline
\end{tabular}

(Table 2. Continued...) 


\begin{tabular}{|c|c|c|c|c|}
\hline \multirow{2}{*}{$\begin{array}{c}\text { Q-class }(P), \\
\text { GAPID, } \\
\text { CARAT index }\end{array}$} & \multirow{2}{*}{$\begin{array}{c}\mathbb{Z}- \\
\text { class } \\
(\Lambda)\end{array}$} & \multirow{2}{*}{$\begin{array}{l}\text { affine } \\
\text { class, } \\
\text { breaking }\end{array}$} & generators of $G$ & \multirow[b]{2}{*}{$\left(h^{(1,1)}, h^{(2,1)}\right)$} \\
\hline & & & $\begin{array}{l}\text { contributions to }\left(h^{(1,1)}, h^{(2,1)}\right) \\
\text { from } U \text { and } T \text { sectors }\end{array}$ & \\
\hline & \multirow[t]{4}{*}{3} & \multirow{2}{*}{$\begin{array}{c}1 \\
\text { local }\end{array}$} & $(\vartheta, 0),(\omega, 0)$ & \multirow[b]{2}{*}{$(24,0)$} \\
\hline & & & $\begin{array}{l}(2,0) U+(4,0) T_{[\vartheta]}+(3,0) T_{[\omega]}+(7,0) T_{\left[\vartheta^{2}\right]}+ \\
(4,0) T_{[\vartheta \omega]}+(1,0) T_{\left[\vartheta^{2} \omega\right]}+(3,0) T_{\left[\vartheta^{4}\right]}\end{array}$ & \\
\hline & & \multirow{2}{*}{$\begin{array}{c}2 \\
\text { local }\end{array}$} & $\left(\vartheta, \frac{1}{2} e_{1}\right),\left(\omega, \frac{1}{2}\left(e_{1}+e_{3}+e_{4}+e_{6}\right)\right)$ & \multirow[b]{2}{*}{$(19,1)$} \\
\hline & & & $\begin{array}{l}(2,0) U+(4,0) T_{[\vartheta]}+(1,1) T_{[\omega]}+(6,0) T_{\left[\vartheta^{2}\right]}+ \\
(4,0) T_{[\vartheta \omega]}+(2,0) T_{\left[\vartheta^{4}\right]}\end{array}$ & \\
\hline & \multirow[t]{8}{*}{4} & \multirow{2}{*}{$\begin{array}{c}1 \\
\text { local }\end{array}$} & $(\vartheta, 0),(\omega, 0)$ & \multirow[b]{2}{*}{$(42,0)$} \\
\hline & & & $\begin{array}{l}(2,0) U+(4,0) T_{[\vartheta]}+(12,0) T_{[\omega]}+(10,0) T_{\left[\vartheta^{2}\right]}+ \\
(4,0) T_{[\vartheta \omega]}+(4,0) T_{\left[\vartheta^{2} \omega\right]}+(6,0) T_{\left[\vartheta^{4}\right]}\end{array}$ & \\
\hline & & \multirow{2}{*}{$\begin{array}{c}2 \\
\text { local }\end{array}$} & $\left(\vartheta, \frac{1}{2}\left(e_{3}+e_{4}\right)\right),\left(\omega, \frac{1}{2}\left(e_{1}+e_{2}+e_{3}+e_{4}\right)\right)$ & \multirow[b]{2}{*}{$(22,4)$} \\
\hline & & & $\begin{array}{l}(2,0) U+(4,0) T_{[\vartheta]}+(6,0) T_{\left[\vartheta^{2}\right]}+(4,0) T_{[\vartheta \omega]}+ \\
(2,2) T_{\left[\vartheta^{2} \omega\right]}+(4,2) T_{\left[\vartheta^{4}\right]}\end{array}$ & \\
\hline & & \multirow{2}{*}{$\begin{array}{c}3 \\
\text { local }\end{array}$} & $(\vartheta, 0),\left(\omega, \frac{1}{2}\left(e_{5}+e_{6}\right)\right)$ & \multirow[b]{2}{*}{$(30,0)$} \\
\hline & & & $\begin{array}{l}(2,0) U+(4,0) T_{[\vartheta]}+(8,0) T_{[\omega]}+(6,0) T_{\left[\vartheta^{2}\right]}+ \\
(4,0) T_{[\vartheta \omega]}+(6,0) T_{\left[\vartheta^{4}\right]}\end{array}$ & \\
\hline & & \multirow{2}{*}{$\begin{array}{c}4 \\
\text { local }\end{array}$} & $\left(\vartheta, \frac{1}{2}\left(e_{3}+e_{4}\right)\right),\left(\omega, \frac{1}{2}\left(e_{1}+e_{2}+e_{3}+e_{4}+e_{5}+e_{6}\right)\right)$ & \multirow[b]{2}{*}{$(20,2)$} \\
\hline & & & $\begin{array}{l}(2,0) U+(4,0) T_{[\vartheta]}+(6,0) T_{\left[\vartheta^{2}\right]}+(4,0) T_{[\vartheta \omega]}+ \\
(4,2) T_{\left[\vartheta^{4}\right]}\end{array}$ & \\
\hline & \multirow[t]{6}{*}{5} & \multirow{2}{*}{$\begin{array}{c}1 \\
\text { local }\end{array}$} & $(\vartheta, 0),(\omega, 0)$ & \multirow[b]{2}{*}{$(30,0)$} \\
\hline & & & $\begin{array}{l}(2,0) U+(4,0) T_{[\vartheta]}+(6,0) T_{[\omega]}+(8,0) T_{\left[\vartheta^{2}\right]}+ \\
(4,0) T_{[\vartheta \omega]}+(2,0) T_{\left[\vartheta^{2} \omega\right]}+(4,0) T_{\left[\vartheta^{4}\right]}\end{array}$ & \\
\hline & & \multirow{2}{*}{$\begin{array}{c}2 \\
\text { local }\end{array}$} & $\left(\vartheta, \frac{1}{2}\left(e_{1}+e_{2}+e_{3}+e_{4}\right)\right),\left(\omega, \frac{1}{2}\left(e_{1}+e_{6}\right)\right)$ & \multirow[b]{2}{*}{$(30,0)$} \\
\hline & & & $\begin{array}{l}(2,0) U+(4,0) T_{[\vartheta]}+(6,0) T_{[\omega]}+(8,0) T_{\left[\vartheta^{2}\right]}+ \\
(4,0) T_{[\vartheta \omega]}+(2,0) T_{\left[\vartheta^{2} \omega\right]}+(4,0) T_{\left[\vartheta^{4}\right]}\end{array}$ & \\
\hline & & \multirow{2}{*}{$\begin{array}{c}3 \\
\text { local }\end{array}$} & $\left(\vartheta, \frac{1}{2}\left(e_{1}+e_{2}\right)\right),\left(\omega, \frac{1}{2}\left(e_{1}+e_{2}+e_{3}+e_{4}+e_{5}\right)\right)$ & \multirow[b]{2}{*}{$(19,1)$} \\
\hline & & & $\begin{array}{l}(2,0) U+(4,0) T_{[\vartheta]}+(6,0) T_{\left[\vartheta^{2}\right]}+(4,0) T_{[\vartheta \omega]}+ \\
(3,1) T_{\left[\vartheta^{4}\right]}\end{array}$ & \\
\hline & \multirow[t]{4}{*}{6} & \multirow{2}{*}{$\begin{array}{c}1 \\
\text { local }\end{array}$} & $(\vartheta, 0),(\omega, 0)$ & \multirow[b]{2}{*}{$(25,1)$} \\
\hline & & & $\begin{array}{l}(2,0) U+(4,0) T_{[\vartheta]}+(4,1) T_{[\omega]}+(7,0) T_{\left[\vartheta^{2}\right]}+ \\
(4,0) T_{[\vartheta \omega]}+(1,0) T_{\left[\vartheta^{2} \omega\right]}+(3,0) T_{\left[\vartheta^{4}\right]}\end{array}$ & \\
\hline & & \multirow{2}{*}{$\begin{array}{c}2 \\
\text { local }\end{array}$} & $\left(\vartheta, \frac{1}{2}\left(e_{1}+e_{2}+e_{4}\right)\right),\left(\omega, \frac{1}{2}\left(e_{1}+e_{4}+e_{5}+e_{6}\right)\right)$ & \multirow[b]{2}{*}{$(18,0)$} \\
\hline & & & $\begin{array}{l}(2,0) U+(4,0) T_{[\vartheta]}+(6,0) T_{\left[\vartheta^{2}\right]}+(4,0) T_{[\vartheta \omega]}+ \\
(2,0) T_{\left[\vartheta^{4}\right]}\end{array}$ & \\
\hline \multirow{6}{*}{$\begin{array}{l}Q D_{16} \\
{[16,8]}\end{array}$} & \multirow[t]{6}{*}{1} & 1 & $(\vartheta, 0),(\omega, 0)$ & \\
\hline & & local & $\begin{array}{l}(2,1) U+(4,4) T_{[\vartheta]}+(4,0) T_{[\omega]}+(5,2) T_{\left[\vartheta^{2}\right]}+ \\
(8,0) T_{[\vartheta \omega]}+(3,1) T_{[\vartheta \omega \vartheta \omega]}\end{array}$ & $(26,8)$ \\
\hline & & 2 & $\left(\vartheta, \frac{1}{2} e_{6}\right),(\omega, 0)$ & \\
\hline & & local & $\begin{array}{l}(2,1) U+(4,0) T_{[\omega]}+(5,2) T_{\left[\vartheta^{2}\right]}+(8,0) T_{[\vartheta \omega]}+ \\
(3,1) T_{[\vartheta \omega \vartheta \omega]}\end{array}$ & $(22,4)$ \\
\hline & & 3 & $\left(\vartheta, \frac{1}{2} e_{1}\right),(\omega, 0)$ & \\
\hline & & local & $\begin{array}{l}(2,1) U+(4,4) T_{[\vartheta]}+(4,0) T_{[\omega]}+(5,2) T_{\left[\vartheta^{2}\right]}+ \\
(8,0) T_{[\vartheta \omega]}+(3,1) T_{[\vartheta \omega \vartheta \omega]}\end{array}$ & $(26,8)$ \\
\hline
\end{tabular}

(Table 2. Continued...) 


\begin{tabular}{|c|c|c|c|c|}
\hline \multirow{2}{*}{$\begin{array}{l}\text { Q-class }(P) \\
\text { GAPID, } \\
\text { CARAT index }\end{array}$} & \multirow{2}{*}{$\begin{array}{l}\mathbb{Z}- \\
\text { class } \\
(\Lambda)\end{array}$} & \multirow{2}{*}{$\begin{array}{l}\text { affine } \\
\text { class, } \\
\text { breaking }\end{array}$} & generators of $G$ & \multirow[b]{2}{*}{$\left(h^{(1,1)}, h^{(2,1)}\right)$} \\
\hline & & & $\begin{array}{l}\text { contributions to }\left(h^{(1,1)}, h^{(2,1)}\right) \\
\text { from } U \text { and } T \text { sectors }\end{array}$ & \\
\hline & & \multirow{2}{*}{$\begin{array}{c}4 \\
\text { local }\end{array}$} & $\left(\vartheta, \frac{1}{2}\left(e_{1}+e_{6}\right)\right),(\omega, 0)$ & \multirow[b]{2}{*}{$(22,4)$} \\
\hline & & & $\begin{array}{l}(2,1) U+(4,0) T_{[\omega]}+(5,2) T_{\left[\vartheta^{2}\right]}+(8,0) T_{[\vartheta \omega]}+ \\
(3,1) T_{[\vartheta \omega \vartheta \omega]}\end{array}$ & \\
\hline & \multirow[t]{6}{*}{2} & \multirow{2}{*}{$\begin{array}{c}1 \\
\text { local }\end{array}$} & $(\vartheta, 0),(\omega, 0)$ & \multirow[b]{2}{*}{$(22,4)$} \\
\hline & & & $\begin{array}{l}(2,1) U+(2,2) T_{[\vartheta]}+(4,0) T_{[\omega]}+(4,1) T_{\left[\vartheta^{2}\right]}+ \\
(8,0) T_{[\vartheta \omega]}+(2,0) T_{[\vartheta \omega \vartheta \omega]}\end{array}$ & \\
\hline & & \multirow{2}{*}{$\begin{array}{c}2 \\
\text { local }\end{array}$} & $\left(\vartheta, \frac{1}{2} e_{1}\right),(\omega, 0)$ & \multirow[b]{2}{*}{$(20,2)$} \\
\hline & & & $\begin{array}{l}(2,1) U+(4,0) T_{[\omega]}+(4,1) T_{\left[\vartheta^{2}\right]}+(8,0) T_{[\vartheta \omega]}+ \\
(2,0) T_{[\vartheta \omega \vartheta \omega]}\end{array}$ & \\
\hline & & \multirow{2}{*}{$\begin{array}{c}3 \\
\text { local }\end{array}$} & $\left(\vartheta, \frac{1}{2}\left(e_{2}+e_{3}+e_{6}\right)\right),(\omega, 0)$ & \multirow[b]{2}{*}{$(19,1)$} \\
\hline & & & $\begin{array}{l}(2,1) U+(4,0) T_{[\omega]}+(3,0) T_{\left[\vartheta^{2}\right]}+(8,0) T_{[\vartheta \omega]}+ \\
(2,0) T_{[\vartheta \omega \vartheta \omega]}\end{array}$ & \\
\hline & \multirow[t]{8}{*}{3} & \multirow{2}{*}{$\begin{array}{c}1 \\
\text { local }\end{array}$} & $(\vartheta, 0),(\omega, 0)$ & \multirow[b]{2}{*}{$(31,7)$} \\
\hline & & & $\begin{array}{l}(2,1) U+(4,4) T_{[\vartheta]}+(8,0) T_{[\omega]}+(6,1) T_{\left[\vartheta^{2}\right]}+ \\
(8,0) T_{[\vartheta \omega]}+(3,1) T_{[\vartheta \omega \vartheta \omega]}\end{array}$ & \\
\hline & & \multirow{2}{*}{$\begin{array}{c}2 \\
\text { local }\end{array}$} & $\left(\vartheta, \frac{1}{2} e_{6}\right),(\omega, 0)$ & \multirow[b]{2}{*}{$(27,3)$} \\
\hline & & & $\begin{array}{l}(2,1) U+(8,0) T_{[\omega]}+(6,1) T_{\left[\vartheta^{2}\right]}+(8,0) T_{[\vartheta \omega]}+ \\
(3,1) T_{[\vartheta \omega \vartheta \omega]}\end{array}$ & \\
\hline & & \multirow{2}{*}{$\begin{array}{c}3 \\
\text { local }\end{array}$} & $\left(\vartheta, \frac{1}{2}\left(e_{1}+e_{2}\right)\right),\left(\omega, \frac{1}{2} e_{1}\right)$ & \multirow[b]{2}{*}{$(21,9)$} \\
\hline & & & $\begin{array}{l}(2,1) U+(4,4) T_{[\vartheta]}+(4,3) T_{\left[\vartheta^{2}\right]}+(8,0) T_{[\vartheta \omega]}+ \\
(3,1) T_{[\vartheta \omega \vartheta \omega]}\end{array}$ & \\
\hline & & \multirow{2}{*}{$\begin{array}{c}4 \\
\text { non-local }\end{array}$} & $\left(\vartheta, \frac{1}{2}\left(e_{1}+e_{2}+e_{6}\right)\right),\left(\omega, \frac{1}{2} e_{1}\right)$ & \multirow[b]{2}{*}{$(17,5)$} \\
\hline & & & $(2,1) U+(4,3) T_{\left[\vartheta^{2}\right]}+(8,0) T_{[\vartheta \omega]}+(3,1) T_{[\vartheta \omega \vartheta \omega]}$ & \\
\hline & \multirow[t]{6}{*}{4} & \multirow{2}{*}{$\begin{array}{c}1 \\
\text { local }\end{array}$} & $(\vartheta, 0),(\omega, 0)$ & \multirow[b]{2}{*}{$(22,4)$} \\
\hline & & & $\begin{array}{l}(2,1) U+(2,2) T_{[\vartheta]}+(4,0) T_{[\omega]}+(4,1) T_{\left[\vartheta^{2}\right]}+ \\
(8,0) T_{[\vartheta \omega]}+(2,0) T_{[\vartheta \omega \vartheta \omega]}\end{array}$ & \\
\hline & & \multirow{2}{*}{$\begin{array}{c}2 \\
\text { local }\end{array}$} & $\left(\vartheta, \frac{1}{2} e_{1}\right),(\omega, 0)$ & \multirow[b]{2}{*}{$(20,2)$} \\
\hline & & & $\begin{array}{l}(2,1) U+(4,0) T_{[\omega]}+(4,1) T_{\left[\vartheta^{2}\right]}+(8,0) T_{[\vartheta \omega]}+ \\
(2,0) T_{[\vartheta \omega \vartheta \omega]}\end{array}$ & \\
\hline & & \multirow{2}{*}{$\begin{array}{c}3 \\
\text { local }\end{array}$} & $\left(\vartheta, \frac{1}{2}\left(e_{2}+e_{6}\right)\right),(\omega, 0)$ & \multirow[b]{2}{*}{$(22,4)$} \\
\hline & & & $\begin{array}{l}(2,1) U+(2,2) T_{[\vartheta]}+(4,0) T_{[\omega]}+(4,1) T_{\left[\vartheta^{2}\right]}+ \\
(8,0) T_{[\vartheta \omega]}+(2,0) T_{[\vartheta \omega \vartheta \omega]}\end{array}$ & \\
\hline \multirow{4}{*}{$\begin{array}{c}\left(\mathbb{Z}_{4} \times \mathbb{Z}_{2}\right) \rtimes \mathbb{Z}_{2} \\
{[16,13]}\end{array}$} & \multirow[t]{4}{*}{1} & \multirow{2}{*}{$\begin{array}{c}1 \\
\text { local }\end{array}$} & $(\vartheta, 0),(\omega, 0),(\rho, 0)$ & \\
\hline & & & $\begin{array}{l}(2,1) U+(8,0) T_{[\vartheta]}+(8,0) T_{[\omega]}+(16,0) T_{[\rho]}+ \\
(4,0) T_{[\vartheta \omega]}+(4,0) T_{[\vartheta \rho]}+(4,0) T_{[\omega \rho]} \\
+(8,0) T_{\left[\vartheta \omega \rho^{3}\right]}+(7,0) T_{\left[\rho^{2}\right]}\end{array}$ & $(61,1)$ \\
\hline & & \multirow{2}{*}{$\begin{array}{c}2 \\
\text { local }\end{array}$} & $\left(\vartheta, \frac{1}{2} e_{5}\right),(\omega, 0),\left(\rho, \frac{1}{2} e_{5}\right)$ & \\
\hline & & & $\begin{array}{l}(2,1) U+(8,0) T_{[\vartheta]}+(4,0) T_{[\omega]}+(8,0) T_{[\rho]}+ \\
(4,0) T_{[\vartheta \rho]}+(4,0) T_{\left[\vartheta \omega \rho^{3}\right]}+(7,0) T_{\left[\rho^{2}\right]}\end{array}$ & $(37,1)$ \\
\hline
\end{tabular}

(Table 2. Continued...) 


\begin{tabular}{|c|c|c|c|c|}
\hline \multirow{2}{*}{$\begin{array}{l}\text { Q-class }(P), \\
\text { GAPID, } \\
\text { CARAT index }\end{array}$} & \multirow{2}{*}{$\begin{array}{c}\mathbb{Z} \text { - } \\
\text { class } \\
(\Lambda)\end{array}$} & \multirow{2}{*}{$\begin{array}{c}\text { affine } \\
\text { class, } \\
\text { breaking }\end{array}$} & generators of $G$ & \multirow[b]{2}{*}{$\left(h^{(1,1)}, h^{(2,1)}\right)$} \\
\hline & & & $\begin{array}{l}\text { contributions to }\left(h^{(1,1)}, h^{(2,1)}\right) \\
\text { from } U \text { and } T \text { sectors }\end{array}$ & \\
\hline & \multirow{2}{*}{\multicolumn{2}{|c|}{$\begin{array}{c}3 \\
\text { local }\end{array}$}} & $\left(\vartheta, \frac{1}{2}\left(e_{1}+e_{3}\right)\right),(\omega, 0),\left(\rho, \frac{1}{2}\left(e_{1}+e_{3}\right)\right)$ & \\
\hline & & & $\begin{array}{l}(2,1) U+(8,0) T_{[\omega]}+(8,0) T_{[\rho]}+(2,2) T_{[\vartheta \omega]}+ \\
(2,2) T_{[\vartheta \rho]}+(4,0) T_{[\omega \rho]}+(4,0) T_{\left[\vartheta \omega \rho^{3}\right]} \\
+(6,1) T_{\left[\rho^{2}\right]}\end{array}$ & $(36,6)$ \\
\hline & & \multirow{2}{*}{$\begin{array}{c}4 \\
\text { local }\end{array}$} & $\left(\vartheta, \frac{1}{2}\left(e_{1}+e_{3}+e_{5}\right)\right),(\omega, 0),\left(\rho, \frac{1}{2}\left(e_{1}+e_{3}+e_{5}\right)\right)$ & \multirow[b]{2}{*}{$(22,4)$} \\
\hline & & & $\begin{array}{l}(2,1) U+(4,0) T_{[\omega]}+(4,0) T_{[\rho]}+(2,2) T_{[\vartheta \rho]}+ \\
(4,0) T_{\left[\vartheta \omega \rho^{3}\right]}+(6,1) T_{\left[\rho^{2}\right]}\end{array}$ & \\
\hline & & \multirow{2}{*}{$\begin{array}{c}5 \\
\text { local }\end{array}$} & $\left(\vartheta, \frac{1}{2} e_{1}\right),(\omega, 0),\left(\rho, \frac{1}{2}\left(e_{1}+e_{4}\right)\right)$ & \multirow[b]{2}{*}{$(31,7)$} \\
\hline & & & $\begin{array}{l}(2,1) U+(8,0) T_{[\omega]}+(8,0) T_{[\rho]}+(2,2) T_{[\vartheta \omega]}+ \\
(2,2) T_{[\vartheta \rho]}+(4,0) T_{[\omega \rho]}+(5,2) T_{\left[\rho^{2}\right]}\end{array}$ & \\
\hline & & \multirow{2}{*}{$\begin{array}{c}6 \\
\text { local }\end{array}$} & $\left(\vartheta, \frac{1}{2}\left(e_{1}+e_{5}\right)\right),(\omega, 0),\left(\rho, \frac{1}{2}\left(e_{1}+e_{4}+e_{5}\right)\right)$ & \multirow[b]{2}{*}{$(17,5)$} \\
\hline & & & $\begin{array}{l}(2,1) U+(4,0) T_{[\omega]}+(4,0) T_{[\rho]}+(2,2) T_{[\vartheta \rho]}+ \\
(5,2) T_{\left[\rho^{2}\right]}\end{array}$ & \\
\hline & & \multirow{2}{*}{$\begin{array}{c}7 \\
\text { local }\end{array}$} & $\left(\vartheta, \frac{1}{2}\left(e_{3}+e_{5}\right)\right),(\omega, 0),\left(\rho, \frac{1}{2}\left(e_{3}+e_{4}+e_{5}\right)\right)$ & \multirow[b]{2}{*}{$(22,4)$} \\
\hline & & & $\begin{array}{l}(2,1) U+(4,0) T_{[\vartheta]}+(4,0) T_{[\omega]}+(4,0) T_{[\rho]}+ \\
(2,2) T_{[\vartheta \rho]}+(6,1) T_{\left[\rho^{2}\right]}\end{array}$ & \\
\hline & & \multirow{2}{*}{$\begin{array}{c}8 \\
\text { local }\end{array}$} & $\left(\vartheta, \frac{1}{2} e_{6}\right),(\omega, 0),\left(\rho, \frac{1}{2}\left(e_{5}+e_{6}\right)\right)$ & \multirow[b]{2}{*}{$(25,1)$} \\
\hline & & & $\begin{array}{l}(2,1) U+(4,0) T_{[\vartheta]}+(4,0) T_{[\omega]}+(4,0) T_{[\rho]}+ \\
(4,0) T_{\left[\vartheta \omega \rho^{3}\right]}+(7,0) T_{\left[\rho^{2}\right]}\end{array}$ & \\
\hline & & \multirow{2}{*}{$\begin{array}{c}9 \\
\text { local }\end{array}$} & $\left(\vartheta, \frac{1}{2}\left(e_{1}+e_{3}+e_{5}\right)\right),(\omega, 0),\left(\rho, \frac{1}{2}\left(e_{1}+e_{3}\right)\right)$ & \multirow[b]{2}{*}{$(32,2)$} \\
\hline & & & $\begin{array}{l}(2,1) U+(8,0) T_{[\omega]}+(8,0) T_{[\rho]}+(4,0) T_{[\omega \rho]}+ \\
(4,0) T_{\left[\vartheta \omega \rho^{3}\right]}+(6,1) T_{\left[\rho^{2}\right]}\end{array}$ & \\
\hline & & \multirow[b]{2}{*}{ local } & $\begin{array}{l}\left(\vartheta, \frac{1}{2}\left(e_{1}+e_{3}+e_{6}\right)\right),(\omega, 0),\left(\rho, \frac{1}{2}\left(e_{1}+e_{3}+e_{5}+\right.\right. \\
\left.\left.e_{6}\right)\right)\end{array}$ & \multirow[b]{2}{*}{$(20,2)$} \\
\hline & & & $\begin{array}{l}(2,1) U+(4,0) T_{[\omega]}+(4,0) T_{[\rho]}+(4,0) T_{\left[\vartheta \omega \rho^{3}\right]}+ \\
(6,1) T_{\left[\rho^{2}\right]}\end{array}$ & \\
\hline & & \multirow{2}{*}{$\begin{array}{c}11 \\
\text { non-local }\end{array}$} & $\left(\vartheta, \frac{1}{2}\left(e_{1}+e_{5}\right)\right),(\omega, 0),\left(\rho, \frac{1}{2}\left(e_{1}+e_{4}\right)\right)$ & \multirow[b]{2}{*}{$(27,3)$} \\
\hline & & & $\begin{array}{l}(2,1) U+(8,0) T_{[\omega]}+(8,0) T_{[\rho]}+(4,0) T_{[\omega \rho]}+ \\
(5,2) T_{\left[\rho^{2}\right]}\end{array}$ & \\
\hline & & \multirow{2}{*}{$\begin{array}{c}12 \\
\text { non-local } \\
\end{array}$} & $\left(\vartheta, \frac{1}{2}\left(e_{1}+e_{6}\right)\right),(\omega, 0),\left(\rho, \frac{1}{2}\left(e_{1}+e_{4}+e_{5}+e_{6}\right)\right)$ & \multirow[b]{2}{*}{$(15,3)$} \\
\hline & & & $(2,1) U+(4,0) T_{[\omega]}+(4,0) T_{[\rho]}+(5,2) T_{\left[\rho^{2}\right]}$ & \\
\hline & & \multirow{2}{*}{$\begin{array}{c}13 \\
\text { local }\end{array}$} & $\left(\vartheta, \frac{1}{2}\left(e_{2}+e_{4}\right)\right),(\omega, 0),\left(\rho, \frac{1}{2}\left(e_{2}+e_{4}\right)\right)$ & \multirow[b]{2}{*}{$(31,7)$} \\
\hline & & & $\begin{array}{l}(2,1) U+(4,0) T_{[\vartheta]}+(4,0) T_{[\omega]}+(4,0) T_{[\rho]}+ \\
(2,2) T_{[\vartheta \omega]}+(2,2) T_{[\vartheta \rho]}+(2,2) T_{[\omega \rho]} \\
+(4,0) T_{\left[\vartheta \omega \rho^{3}\right]}+(7,0) T_{\left[\rho^{2}\right]}\end{array}$ & \\
\hline & & \multirow{2}{*}{$\begin{array}{c}14 \\
\text { local }\end{array}$} & $\left(\vartheta, \frac{1}{2}\left(e_{2}+e_{4}+e_{5}\right)\right),(\omega, 0),\left(\rho, \frac{1}{2}\left(e_{2}+e_{4}+e_{5}\right)\right)$ & \multirow[b]{2}{*}{$(27,3)$} \\
\hline & & & $\begin{array}{l}(2,1) U+(4,0) T_{[\vartheta]}+(4,0) T_{[\omega]}+(4,0) T_{[\rho]}+ \\
(2,2) T_{[\vartheta \rho]}+(4,0) T_{\left[\vartheta \omega \rho^{3}\right]}+(7,0) T_{\left[\rho^{2}\right]}\end{array}$ & \\
\hline & & \multirow{2}{*}{$\begin{array}{c}15 \\
\text { local }\end{array}$} & $\left(\vartheta, \frac{1}{2}\left(e_{1}+e_{2}+e_{4}\right)\right),(\omega, 0),\left(\rho, \frac{1}{2}\left(e_{1}+e_{2}\right)\right)$ & \multirow[b]{2}{*}{$(21,9)$} \\
\hline & & & $\begin{array}{l}(2,1) U+(4,0) T_{[\omega]}+(4,0) T_{[\rho]}+(2,2) T_{[\vartheta \omega]}+ \\
(2,2) T_{[\vartheta \rho]}+(2,2) T_{[\omega \rho]}+(5,2) T_{\left[\rho^{2}\right]}\end{array}$ & \\
\hline
\end{tabular}

(Table 2. Continued...) 


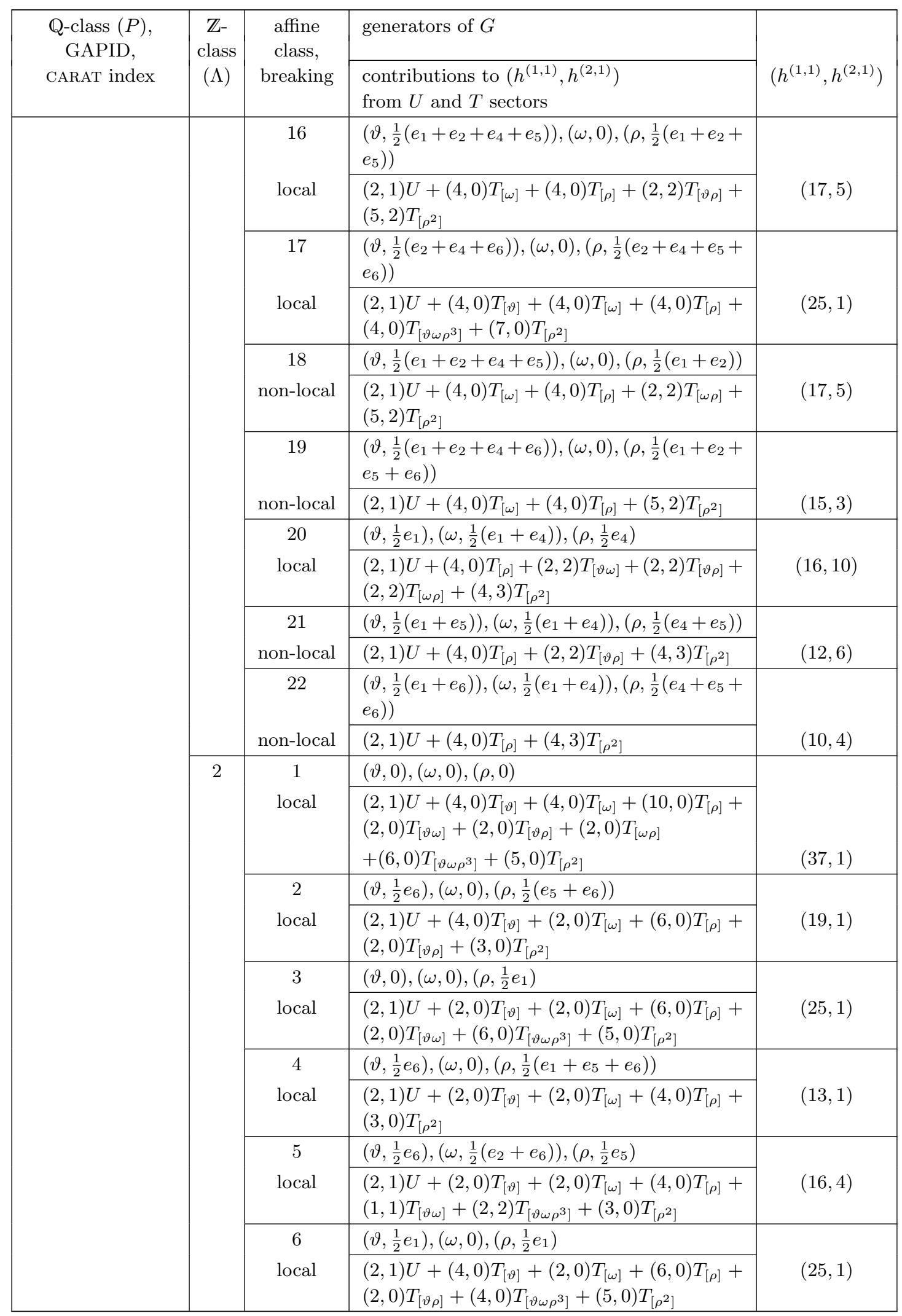

(Table 2. Continued...) 


\begin{tabular}{|c|c|c|c|c|}
\hline \multirow{2}{*}{$\begin{array}{l}\text { Q-class }(P), \\
\text { GAPID, } \\
\text { CARAT index }\end{array}$} & \multirow{2}{*}{$\begin{array}{c}\mathbb{Z}^{-} \\
\text {class } \\
(\Lambda)\end{array}$} & \multirow{2}{*}{$\begin{array}{c}\text { affine } \\
\text { class, } \\
\text { breaking }\end{array}$} & generators of $G$ & \multirow[b]{2}{*}{$\left(h^{(1,1)}, h^{(2,1)}\right)$} \\
\hline & & & $\begin{array}{l}\text { contributions to }\left(h^{(1,1)}, h^{(2,1)}\right) \\
\text { from } U \text { and } T \text { sectors }\end{array}$ & \\
\hline & & \multirow{2}{*}{$\begin{array}{c}7 \\
\text { local }\end{array}$} & $\left(\vartheta, \frac{1}{2}\left(e_{1}+e_{6}\right)\right),\left(\omega, \frac{1}{2}\left(e_{2}+e_{6}\right)\right),\left(\rho, \frac{1}{2}\left(e_{1}+e_{5}\right)\right)$ & \multirow[b]{2}{*}{$(15,3)$} \\
\hline & & & $\begin{array}{l}(2,1) U+(2,0) T_{[\vartheta]}+(2,0) T_{[\omega]}+(4,0) T_{[\rho]}+ \\
(2,2) T_{\left[\vartheta \omega \rho^{3}\right]}+(3,0) T_{\left[\rho^{2}\right]}\end{array}$ & \\
\hline & \multirow[t]{8}{*}{3} & \multirow{2}{*}{$\begin{array}{c}1 \\
\text { local }\end{array}$} & $(\vartheta, 0),(\omega, 0),(\rho, 0)$ & \multirow[b]{2}{*}{$(25,1)$} \\
\hline & & & $\begin{array}{l}(2,1) U+(3,0) T_{[\vartheta]}+(3,0) T_{[\omega]}+(7,0) T_{[\rho]}+ \\
(1,0) T_{[\vartheta \omega]}+(1,0) T_{[\vartheta \rho]}+(1,0) T_{[\omega \rho]} \\
+(3,0) T_{\left[\vartheta \omega \rho^{3}\right]}+(4,0) T_{\left[\rho^{2}\right]}\end{array}$ & \\
\hline & & \multirow{2}{*}{$\begin{array}{c}2 \\
\text { local }\end{array}$} & $\left(\vartheta, \frac{1}{2}\left(e_{2}+e_{6}\right)\right),(\omega, 0),\left(\rho, \frac{1}{2}\left(e_{2}+e_{6}\right)\right)$ & \multirow[b]{2}{*}{$(25,1)$} \\
\hline & & & $\begin{array}{l}(2,1) U+(3,0) T_{[\vartheta]}+(3,0) T_{[\omega]}+(7,0) T_{[\rho]}+ \\
(1,0) T_{[\vartheta \omega]}+(1,0) T_{[\vartheta \rho]}+(1,0) T_{[\omega \rho]} \\
+(3,0) T_{\left[\vartheta \omega \rho^{3}\right]}+(4,0) T_{\left[\rho^{2}\right]}\end{array}$ & \\
\hline & & \multirow[t]{2}{*}{$\begin{array}{c}3 \\
\text { local }\end{array}$} & $\begin{array}{l}\left(\vartheta, \frac{1}{4}\left(e_{1}+2 e_{2}+2 e_{3}+3 e_{4}+3 e_{5}+3 e_{6}\right)\right),(\omega, 0) \\
\left(\rho, \frac{1}{4}\left(e_{1}+3 e_{2}+e_{3}+2 e_{4}+3 e_{6}\right)\right)\end{array}$ & \multirow[b]{2}{*}{$(10,4)$} \\
\hline & & & $\begin{array}{l}(2,1) U+(1,1) T_{[\vartheta]}+(1,1) T_{[\omega]}+(4,0) T_{[\rho]}+ \\
(1,1) T_{\left[\vartheta \omega \rho^{3}\right]}+(1,0) T_{\left[\rho^{2}\right]}\end{array}$ & \\
\hline & & \multirow[b]{2}{*}{ local } & $\begin{array}{l}\left(\vartheta, \frac{1}{2}\left(e_{1}+e_{4}+e_{5}+e_{6}\right)\right),(\omega, 0),\left(\rho, \frac{1}{2}\left(e_{1}+e_{2}+\right.\right. \\
\left.\left.e_{3}+e_{6}\right)\right)\end{array}$ & \multirow[b]{2}{*}{$(25,1)$} \\
\hline & & & $\begin{array}{l}(2,1) U+(3,0) T_{[\vartheta]}+(3,0) T_{[\omega]}+(7,0) T_{[\rho]}+ \\
(1,0) T_{[\vartheta \omega]}+(1,0) T_{[\vartheta \rho]}+(1,0) T_{[\omega \rho]} \\
+(3,0) T_{\left[\vartheta \omega \rho^{3}\right]}+(4,0) T_{\left[\rho^{2}\right]}\end{array}$ & \\
\hline & \multirow[t]{12}{*}{4} & \multirow{2}{*}{$\begin{array}{c}1 \\
\text { local }\end{array}$} & $(\vartheta, 0),(\omega, 0),(\rho, 0)$ & \multirow[b]{2}{*}{$(51,3)$} \\
\hline & & & $\begin{array}{l}(2,1) U+(4,0) T_{[\vartheta]}+(4,0) T_{[\omega]}+(12,0) T_{[\rho]}+ \\
(4,0) T_{[\vartheta \omega]}+(3,1) T_{[\vartheta \rho]}+(3,1) T_{[\omega \rho]} \\
+(12,0) T_{\left[\vartheta \omega \rho^{3}\right]}+(7,0) T_{\left[\rho^{2}\right]}\end{array}$ & \\
\hline & & \multirow{2}{*}{$\begin{array}{c}2 \\
\text { local }\end{array}$} & $\left(\vartheta, \frac{1}{2}\left(e_{1}+e_{2}\right)\right),(\omega, 0),\left(\rho, \frac{1}{2}\left(e_{1}+e_{4}\right)\right)$ & \multirow[b]{2}{*}{$(31,7)$} \\
\hline & & & $\begin{array}{l}(2,1) U+(4,0) T_{[\vartheta]}+(4,0) T_{[\omega]}+(8,0) T_{[\rho]}+ \\
(2,2) T_{[\vartheta \omega]}+(3,1) T_{[\vartheta \rho]}+(3,1) T_{[\omega \rho]} \\
+(5,2) T_{\left[\rho^{2}\right]}\end{array}$ & \\
\hline & & 3 & $(\vartheta, 0),(\omega, 0),\left(\rho, \frac{1}{2} e_{5}\right)$ & \multirow[b]{2}{*}{$(37,1)$} \\
\hline & & local & $\begin{array}{l}(2,1) U+(2,0) T_{[\vartheta]}+(2,0) T_{[\omega]}+(8,0) T_{[\rho]}+ \\
(4,0) T_{[\vartheta \omega]}+(12,0) T_{\left[\vartheta \omega \rho^{3}\right]}+(7,0) T_{\left[\rho^{2}\right]}\end{array}$ & \\
\hline & & \multirow{2}{*}{$\begin{array}{c}4 \\
\text { local }\end{array}$} & $\left(\vartheta, \frac{1}{2}\left(e_{1}+e_{2}\right)\right),(\omega, 0),\left(\rho, \frac{1}{2}\left(e_{1}+e_{4}+e_{5}\right)\right)$ & \multirow[b]{2}{*}{$(17,5)$} \\
\hline & & & $\begin{array}{l}(2,1) U+(2,0) T_{[\vartheta]}+(2,0) T_{[\omega]}+(4,0) T_{[\rho]}+ \\
(2,2) T_{[\vartheta \omega]}+(5,2) T_{\left[\rho^{2}\right]}\end{array}$ & \\
\hline & & \multirow{2}{*}{$\begin{array}{c}5 \\
\text { local }\end{array}$} & $\left(\vartheta, \frac{1}{2} e_{6}\right),(\omega, 0),\left(\rho, \frac{1}{2} e_{6}\right)$ & \multirow[b]{2}{*}{$(32,2)$} \\
\hline & & & $\begin{array}{l}(2,1) U+(4,0) T_{[\vartheta]}+(2,0) T_{[\omega]}+(6,0) T_{[\rho]}+ \\
(3,1) T_{[\vartheta \rho]}+(8,0) T_{\left[\vartheta \omega \rho^{3}\right]}+(7,0) T_{\left[\rho^{2}\right]} \\
\end{array}$ & \\
\hline & & \multirow{2}{*}{$\begin{array}{c}6 \\
\text { local }\end{array}$} & $\left(\vartheta, \frac{1}{2}\left(e_{1}+e_{2}+e_{6}\right)\right),(\omega, 0),\left(\rho, \frac{1}{2}\left(e_{1}+e_{4}+e_{6}\right)\right)$ & \multirow[b]{2}{*}{$(22,4)$} \\
\hline & & & $\begin{array}{l}(2,1) U+(4,0) T_{[\vartheta]}+(2,0) T_{[\omega]}+(6,0) T_{[\rho]}+ \\
(3,1) T_{[\vartheta \rho]}+(5,2) T_{\left[\rho^{2}\right]}\end{array}$ & \\
\hline
\end{tabular}

(Table 2. Continued...) 


\begin{tabular}{|c|c|c|c|c|}
\hline \multirow{2}{*}{$\begin{array}{l}\text { Q-class }(P), \\
\text { GAPID, } \\
\text { CARAT index }\end{array}$} & \multirow{2}{*}{$\begin{array}{c}\mathbb{Z}^{-} \\
\text {class } \\
(\Lambda)\end{array}$} & \multirow{2}{*}{$\begin{array}{c}\text { affine } \\
\text { class, } \\
\text { breaking }\end{array}$} & generators of $G$ & \multirow[b]{2}{*}{$\left(h^{(1,1)}, h^{(2,1)}\right)$} \\
\hline & & & $\begin{array}{l}\text { contributions to }\left(h^{(1,1)}, h^{(2,1)}\right) \\
\text { from } U \text { and } T \text { sectors }\end{array}$ & \\
\hline & & \multirow{2}{*}{$\begin{array}{c}7 \\
\text { local }\end{array}$} & $\left(\vartheta, \frac{1}{2} e_{6}\right),(\omega, 0),\left(\rho, \frac{1}{2}\left(e_{5}+e_{6}\right)\right)$ & \multirow[b]{2}{*}{$(25,1)$} \\
\hline & & & $\begin{array}{l}(2,1) U+(2,0) T_{[\vartheta]}+(2,0) T_{[\omega]}+(4,0) T_{[\rho]}+ \\
(8,0) T_{\left[\vartheta \omega \rho^{3}\right]}+(7,0) T_{\left[\rho^{2}\right]}\end{array}$ & \\
\hline & & \multirow{2}{*}{$\begin{array}{c}8 \\
\text { local }\end{array}$} & $\begin{array}{l}\left(\vartheta, \frac{1}{2}\left(e_{1}+e_{2}+e_{6}\right)\right),(\omega, 0),\left(\rho, \frac{1}{2}\left(e_{1}+e_{4}+e_{5}+\right.\right. \\
\left.\left.e_{6}\right)\right)\end{array}$ & \multirow[b]{2}{*}{$(15,3)$} \\
\hline & & & $\begin{array}{l}(2,1) U+(2,0) T_{[\vartheta]}+(2,0) T_{[\omega]}+(4,0) T_{[\rho]}+ \\
(5,2) T_{\left[\rho^{2}\right]}\end{array}$ & \\
\hline & \multirow[t]{18}{*}{5} & \multirow{2}{*}{$\begin{array}{c}1 \\
\text { local }\end{array}$} & $(\vartheta, 0),(\omega, 0),(\rho, 0)$ & \multirow[b]{2}{*}{$(37,1)$} \\
\hline & & & $\begin{array}{l}(2,1) U+(4,0) T_{[\vartheta]}+(4,0) T_{[\omega]}+(10,0) T_{[\rho]}+ \\
(2,0) T_{[\vartheta \omega]}+(2,0) T_{[\vartheta \rho]}+(2,0) T_{[\omega \rho]} \\
+(6,0) T_{\left[\vartheta \omega \rho^{3}\right]}+(5,0) T_{\left[\rho^{2}\right]}\end{array}$ & \\
\hline & & \multirow{2}{*}{$\begin{array}{c}2 \\
\text { local }\end{array}$} & $\left(\vartheta, \frac{1}{2} e_{1}\right),(\omega, 0),\left(\rho, \frac{1}{2} e_{1}\right)$ & \multirow[b]{2}{*}{$(25,1)$} \\
\hline & & & $\begin{array}{l}(2,1) U+(4,0) T_{[\vartheta]}+(2,0) T_{[\omega]}+(6,0) T_{[\rho]}+ \\
(2,0) T_{[\vartheta \rho]}+(4,0) T_{\left[\vartheta \omega \rho^{3}\right]}+(5,0) T_{\left[\rho^{2}\right]} \\
\end{array}$ & \\
\hline & & \multirow[b]{2}{*}{ local } & $\begin{array}{l}\left(\vartheta, \frac{1}{2}\left(e_{2}+e_{3}+e_{4}+e_{5}\right)\right),(\omega, 0),\left(\rho, \frac{1}{2}\left(e_{2}+e_{3}+\right.\right. \\
\left.\left.e_{4}+e_{5}\right)\right)\end{array}$ & \multirow[b]{2}{*}{$(27,3)$} \\
\hline & & & $\begin{array}{l}(2,1) U+(2,0) T_{[\vartheta]}+(2,0) T_{[\omega]}+(6,0) T_{[\rho]}+ \\
(2,0) T_{[\vartheta \omega]}+(1,1) T_{[\vartheta \rho]}+(1,1) T_{[\omega \rho]} \\
+(6,0) T_{\left[\vartheta \omega \rho^{3}\right]}+(5,0) T_{\left[\rho^{2}\right]}\end{array}$ & \\
\hline & & \multirow[b]{2}{*}{ local } & $\begin{array}{l}\left(\vartheta, \frac{1}{2}\left(e_{1}+e_{2}+e_{3}+e_{4}+e_{5}\right)\right),(\omega, 0),\left(\rho, \frac{1}{2}\left(e_{1}+\right.\right. \\
\left.\left.e_{2}+e_{3}+e_{4}+e_{5}\right)\right)\end{array}$ & \multirow[b]{2}{*}{$(20,2)$} \\
\hline & & & $\begin{array}{l}(2,1) U+(2,0) T_{[\vartheta]}+(2,0) T_{[\omega]}+(4,0) T_{[\rho]}+ \\
(1,1) T_{[\vartheta \rho]}+(4,0) T_{\left[\vartheta \omega \rho^{3}\right]}+(5,0) T_{\left[\rho^{2}\right]}\end{array}$ & \\
\hline & & \multirow{2}{*}{$\begin{array}{c}5 \\
\text { local }\end{array}$} & $\left(\vartheta, \frac{1}{2}\left(e_{3}+e_{4}\right)\right),(\omega, 0),\left(\rho, \frac{1}{2}\left(e_{2}+e_{3}\right)\right)$ & \multirow[b]{2}{*}{$(22,4)$} \\
\hline & & & $\begin{array}{l}(2,1) U+(4,0) T_{[\vartheta]}+(2,0) T_{[\omega]}+(6,0) T_{[\rho]}+ \\
(1,1) T_{[\vartheta \omega]}+(2,0) T_{[\vartheta \rho]}+(1,1) T_{[\omega \rho]} \\
+(4,1) T_{\left[\rho^{2}\right]}\end{array}$ & \\
\hline & & \multirow{2}{*}{$\begin{array}{c}6 \\
\text { local }\end{array}$} & $\left(\vartheta, \frac{1}{2}\left(e_{1}+e_{3}+e_{4}\right)\right),(\omega, 0),\left(\rho, \frac{1}{2}\left(e_{1}+e_{2}+e_{3}\right)\right)$ & \multirow[b]{2}{*}{$(20,2)$} \\
\hline & & & $\begin{array}{l}(2,1) U+(4,0) T_{[\vartheta]}+(2,0) T_{[\omega]}+(6,0) T_{[\rho]}+ \\
(2,0) T_{[\vartheta \rho]}+(4,1) T_{\left[\rho^{2}\right]}\end{array}$ & \\
\hline & & \multirow{2}{*}{$\begin{array}{c}7 \\
\text { local }\end{array}$} & $\left(\vartheta, \frac{1}{2}\left(e_{1}+e_{2}+e_{5}\right)\right),(\omega, 0),\left(\rho, \frac{1}{2}\left(e_{1}+e_{4}+e_{5}\right)\right)$ & \multirow[b]{2}{*}{$(15,3)$} \\
\hline & & & $\begin{array}{l}(2,1) U+(2,0) T_{[\vartheta]}+(2,0) T_{[\omega]}+(4,0) T_{[\rho]}+ \\
(1,1) T_{[\vartheta \rho]}+(4,1) T_{\left[\rho^{2}\right]}\end{array}$ & \\
\hline & & \multirow{2}{*}{$\begin{array}{c}8 \\
\text { local }\end{array}$} & $\left(\vartheta, \frac{1}{2}\left(e_{2}+e_{6}\right)\right),(\omega, 0),\left(\rho, \frac{1}{2}\left(e_{2}+e_{6}\right)\right)$ & \multirow[b]{2}{*}{$(32,2)$} \\
\hline & & & $\begin{array}{l}(2,1) U+(4,0) T_{[\vartheta]}+(2,0) T_{[\omega]}+(8,0) T_{[\rho]}+ \\
(2,0) T_{[\vartheta \omega]}+(2,0) T_{[\vartheta \rho]}+(1,1) T_{[\omega \rho]} \\
+(6,0) T_{\left[\vartheta \omega \rho^{3}\right]}+(5,0) T_{\left[\rho^{2}\right]}\end{array}$ & \\
\hline & & \multirow{2}{*}{$\begin{array}{c}9 \\
\text { local }\end{array}$} & $\left(\vartheta, \frac{1}{2}\left(e_{2}+e_{3}+e_{4}+e_{6}\right)\right),(\omega, 0),\left(\rho, \frac{1}{2}\left(e_{3}+e_{6}\right)\right)$ & \multirow[b]{2}{*}{$(27,3)$} \\
\hline & & & $\begin{array}{l}(2,1) U+(4,0) T_{[\vartheta]}+(4,0) T_{[\omega]}+(8,0) T_{[\rho]}+ \\
(1,1) T_{[\vartheta \omega]}+(2,0) T_{[\vartheta \rho]}+(2,0) T_{[\omega \rho]} \\
+(4,1) T_{\left[\rho^{2}\right]}\end{array}$ & \\
\hline
\end{tabular}

(Table 2. Continued...) 


\begin{tabular}{|c|c|c|c|c|}
\hline \multirow{2}{*}{$\begin{array}{l}\text { Q-class }(P), \\
\text { GAPID, } \\
\text { CARAT index }\end{array}$} & \multirow{2}{*}{$\begin{array}{c}\mathbb{Z}_{-} \\
\text {class } \\
(\Lambda)\end{array}$} & \multirow{2}{*}{$\begin{array}{c}\text { affine } \\
\text { class, } \\
\text { breaking }\end{array}$} & generators of $G$ & \multirow[b]{2}{*}{$\left(h^{(1,1)}, h^{(2,1)}\right)$} \\
\hline & & & $\begin{array}{l}\text { contributions to }\left(h^{(1,1)}, h^{(2,1)}\right) \\
\text { from } U \text { and } T \text { sectors }\end{array}$ & \\
\hline & & \multirow{2}{*}{$\begin{array}{c}10 \\
\text { local }\end{array}$} & $\left(\vartheta, \frac{1}{2}\left(e_{5}+e_{6}\right)\right),(\omega, 0),\left(\rho, \frac{1}{2}\left(e_{2}+e_{4}+e_{5}+e_{6}\right)\right)$ & \multirow[b]{2}{*}{$(17,5)$} \\
\hline & & & $\begin{array}{l}(2,1) U+(2,0) T_{[\vartheta]}+(2,0) T_{[\omega]}+(4,0) T_{[\rho]}+ \\
(1,1) T_{[\vartheta \omega]}+(1,1) T_{[\vartheta \rho]}+(1,1) T_{[\omega \rho]} \\
+(4,1) T_{\left[\rho^{2}\right]}\end{array}$ & \\
\hline & & \multirow{2}{*}{$\begin{array}{c}11 \\
\text { local }\end{array}$} & $(\vartheta, 0),(\omega, 0),\left(\rho, \frac{1}{2} e_{1}\right)$ & \multirow[b]{2}{*}{$(25,1)$} \\
\hline & & & $\begin{array}{l}(2,1) U+(2,0) T_{[\vartheta]}+(2,0) T_{[\omega]}+(6,0) T_{[\rho]}+ \\
(2,0) T_{[\vartheta \omega]}+(6,0) T_{\left[\vartheta \omega \rho^{3}\right]}+(5,0) T_{\left[\rho^{2}\right]}\end{array}$ & \\
\hline & & \multirow{2}{*}{$\begin{array}{c}12 \\
\text { local }\end{array}$} & $\left(\vartheta, \frac{1}{2}\left(e_{3}+e_{4}\right)\right),(\omega, 0),\left(\rho, \frac{1}{2}\left(e_{1}+e_{2}+e_{3}\right)\right)$ & \multirow[b]{2}{*}{$(15,3)$} \\
\hline & & & $\begin{array}{l}(2,1) U+(2,0) T_{[\vartheta]}+(2,0) T_{[\omega]}+(4,0) T_{[\rho]}+ \\
(1,1) T_{[\vartheta \omega]}+(4,1) T_{\left[\rho^{2}\right]}\end{array}$ & \\
\hline & & \multirow{2}{*}{$\begin{array}{c}13 \\
\text { local }\end{array}$} & $\left(\vartheta, \frac{1}{2}\left(e_{2}+e_{6}\right)\right),(\omega, 0),\left(\rho, \frac{1}{2}\left(e_{1}+e_{2}+e_{6}\right)\right)$ & \multirow[b]{2}{*}{$(25,1)$} \\
\hline & & & $\begin{array}{l}(2,1) U+(2,0) T_{[\vartheta]}+(2,0) T_{[\omega]}+(6,0) T_{[\rho]}+ \\
(2,0) T_{[\vartheta \omega]}+(6,0) T_{\left[\vartheta \omega \rho^{3}\right]}+(5,0) T_{\left[\rho^{2}\right]}\end{array}$ & \\
\hline & & \multirow{2}{*}{$\begin{array}{l}14 \\
\text { local }\end{array}$} & $\begin{array}{l}\left(\vartheta, \frac{1}{2}\left(e_{2}+e_{3}+e_{4}+e_{6}\right)\right),(\omega, 0),\left(\rho, \frac{1}{2}\left(e_{1}+e_{3}+\right.\right. \\
\left.\left.e_{6}\right)\right)\end{array}$ & \multirow[b]{2}{*}{$(15,3)$} \\
\hline & & & $\begin{array}{l}(2,1) U+(2,0) T_{[\vartheta]}+(2,0) T_{[\omega]}+(4,0) T_{[\rho]}+ \\
(1,1) T_{[\vartheta \omega]}+(4,1) T_{\left[\rho^{2}\right]}\end{array}$ & \\
\hline \multirow{16}{*}{$\begin{array}{c}\mathbb{Z}_{3} \times S_{3} \\
{[18,3]}\end{array}$} & \multirow[t]{8}{*}{1} & \multirow{2}{*}{$\begin{array}{c}1 \\
\text { local }\end{array}$} & $(\vartheta, 0),(\omega, 0)$ & \multirow[b]{2}{*}{$(49,1)$} \\
\hline & & & $\begin{array}{l}(2,0) U+(9,0) T_{[\vartheta]}+(3,0) T_{[\omega]}+(15,0) T_{\left[\omega^{2}\right]}+ \\
(9,0) T_{\left[\vartheta^{2} \omega^{2}\right]}+(2,1) T_{\left[\omega^{3}\right]}+(9,0) T_{\left[\vartheta^{2} \omega^{4}\right]}\end{array}$ & \\
\hline & & \multirow{2}{*}{$\begin{array}{c}2 \\
\text { local }\end{array}$} & $(\vartheta, 0),\left(\omega, \frac{1}{3}\left(e_{3}+e_{4}\right)\right)$ & \multirow[b]{2}{*}{$(16,4)$} \\
\hline & & & $\begin{array}{l}(2,0) U+(3,3) T_{[\vartheta]}+(3,0) T_{[\omega]}+(6,0) T_{\left[\omega^{2}\right]}+ \\
(2,1) T_{\left[\omega^{3}\right]}\end{array}$ & \\
\hline & & \multirow{2}{*}{$\begin{array}{c}3 \\
\text { local }\end{array}$} & $\left(\vartheta, \frac{1}{3}\left(e_{5}+e_{6}\right)\right),(\omega, 0)$ & \multirow[b]{2}{*}{$(19,7)$} \\
\hline & & & $\begin{array}{lll}(2,0) U+(3,0) T_{[\omega]}+(6,0) T_{\left[\omega^{2}\right]}+ & + \\
(3,3) T_{\left[\vartheta^{2} \omega^{2}\right]}+(2,1) T_{\left[\omega^{3}\right]}+(3,3) T_{\left[\vartheta^{2} \omega^{4}\right]} & \end{array}$ & \\
\hline & & \multirow{2}{*}{$\begin{array}{c}4 \\
\text { local }\end{array}$} & $\left(\vartheta, \frac{1}{3}\left(e_{5}+e_{6}\right)\right),\left(\omega, \frac{1}{3}\left(e_{3}+e_{4}\right)\right)$ & \multirow[b]{2}{*}{$(13,1)$} \\
\hline & & & $(2,0) U+(3,0) T_{[\omega]}+(6,0) T_{\left[\omega^{2}\right]}+(2,1) T_{\left[\omega^{3}\right]}$ & \\
\hline & \multirow[t]{4}{*}{2} & \multirow{2}{*}{$\begin{array}{c}1 \\
\text { local }\end{array}$} & $(\vartheta, 0),(\omega, 0)$ & \multirow[b]{2}{*}{$(25,1)$} \\
\hline & & & $\begin{array}{l}(2,0) U+(3,0) T_{[\vartheta]}+(3,0) T_{[\omega]}+(9,0) T_{\left[\omega^{2}\right]}+ \\
(3,0) T_{\left[\vartheta^{2} \omega^{2}\right]}+(2,1) T_{\left[\omega^{3}\right]}+(3,0) T_{\left[\vartheta^{2} \omega^{4}\right]}\end{array}$ & \\
\hline & & \multirow{2}{*}{$\begin{array}{c}2 \\
\text { local }\end{array}$} & $\left(\vartheta, \frac{1}{3}\left(2 e_{1}+e_{5}\right)\right),\left(\omega, \frac{1}{3}\left(2 e_{2}+e_{3}\right)\right)$ & \multirow[b]{2}{*}{$(13,1)$} \\
\hline & & & $(2,0) U+(3,0) T_{[\omega]}+(6,0) T_{\left[\omega^{2}\right]}+(2,1) T_{\left[\omega^{3}\right]}$ & \\
\hline & \multirow[t]{4}{*}{3} & \multirow{2}{*}{$\begin{array}{c}1 \\
\text { local }\end{array}$} & $(\vartheta, 0),(\omega, 0)$ & \multirow[b]{2}{*}{$(27,3)$} \\
\hline & & & $\begin{array}{l}(2,0) U+(5,2) T_{[\vartheta]}+(3,0) T_{[\omega]}+(9,0) T_{\left[\omega^{2}\right]}+ \\
(3,0) T_{\left[\vartheta^{2} \omega^{2}\right]}+(2,1) T_{\left[\omega^{3}\right]}+(3,0) T_{\left[\vartheta^{2} \omega^{4}\right]} \\
\end{array}$ & \\
\hline & & \multirow{2}{*}{$\begin{array}{c}2 \\
\text { local }\end{array}$} & $(\vartheta, 0),\left(\omega, \frac{1}{3}\left(e_{1}+e_{3}\right)\right)$ & \\
\hline & & & $\begin{array}{l}(2,0) U+(3,3) T_{[\vartheta]}+(3,0) T_{[\omega]}+(6,0) T_{\left[\omega^{2}\right]}+ \\
(2,1) T_{\left[\omega^{3}\right]}\end{array}$ & $(16,4)$ \\
\hline
\end{tabular}

(Table 2. Continued ...) 


\begin{tabular}{|c|c|c|c|c|}
\hline \multirow{2}{*}{$\begin{array}{l}\text { Q-class }(P), \\
\text { GAPID, } \\
\text { CARAT index }\end{array}$} & \multirow{2}{*}{$\begin{array}{c}\mathbb{Z}- \\
\text { class } \\
(\Lambda)\end{array}$} & \multirow{2}{*}{$\begin{array}{c}\text { affine } \\
\text { class, } \\
\text { breaking }\end{array}$} & generators of $G$ & \multirow[b]{2}{*}{$\left(h^{(1,1)}, h^{(2,1)}\right)$} \\
\hline & & & $\begin{array}{l}\text { contributions to }\left(h^{(1,1)}, h^{(2,1)}\right) \\
\text { from } U \text { and } T \text { sectors }\end{array}$ & \\
\hline & & \multirow{2}{*}{$\begin{array}{c}3 \\
\text { local }\end{array}$} & $\left(\vartheta, \frac{1}{3}\left(2 e_{5}+e_{6}\right)\right),(\omega, 0)$ & \multirow[b]{2}{*}{$(15,3)$} \\
\hline & & & $\begin{array}{l}(2,0) U+(3,0) T_{[\omega]}+(6,0) T_{\left[\omega^{2}\right]}+ \\
(1,1) T_{\left[\vartheta^{2} \omega^{2}\right]}+(2,1) T_{\left[\omega^{3}\right]}+(1,1) T_{\left[\vartheta^{2} \omega^{4}\right]}\end{array}$ & \\
\hline & & \multirow{2}{*}{$\begin{array}{c}4 \\
\text { local } \\
\end{array}$} & $\left(\vartheta, \frac{1}{3}\left(2 e_{5}+e_{6}\right)\right),\left(\omega, \frac{1}{3}\left(e_{1}+e_{3}\right)\right)$ & \multirow[b]{2}{*}{$(13,1)$} \\
\hline & & & $(2,0) U+(3,0) T_{[\omega]}+(6,0) T_{\left[\omega^{2}\right]}+(2,1) T_{\left[\omega^{3}\right]}$ & \\
\hline & \multirow[t]{6}{*}{4} & \multirow{2}{*}{$\begin{array}{c}1 \\
\text { local }\end{array}$} & $(\vartheta, 0),(\omega, 0)$ & \multirow[b]{2}{*}{$(25,1)$} \\
\hline & & & $\begin{array}{l}(2,0) U+(3,0) T_{[\vartheta]}+(3,0) T_{[\omega]}+(9,0) T_{\left[\omega^{2}\right]}+ \\
(3,0) T_{\left[\vartheta^{2} \omega^{2}\right]}+(2,1) T_{\left[\omega^{3}\right]}+(3,0) T_{\left[\vartheta^{2} \omega^{4}\right]}\end{array}$ & \\
\hline & & \multirow{2}{*}{$\begin{array}{c}2 \\
\text { local }\end{array}$} & $(\vartheta, 0),\left(\omega, \frac{1}{3}\left(e_{2}+e_{4}\right)\right)$ & \multirow[b]{2}{*}{$(16,4)$} \\
\hline & & & $\begin{array}{l}(2,0) U+(1,1) T_{[\vartheta]}+(3,0) T_{[\omega]}+(6,0) T_{\left[\omega^{2}\right]}+ \\
(1,1) T_{\left[\vartheta^{2} \omega^{2}\right]}+(2,1) T_{\left[\omega^{3}\right]}+(1,1) T_{\left[\vartheta^{2} \omega^{4}\right]}\end{array}$ & \\
\hline & & \multirow{2}{*}{$\begin{array}{c}3 \\
\text { local }\end{array}$} & $\left(\vartheta, \frac{1}{3}\left(e_{5}+e_{6}\right)\right),\left(\omega, \frac{1}{3}\left(e_{1}+e_{2}\right)\right)$ & \multirow{2}{*}{$(13,1)$} \\
\hline & & & $(2,0) U+(3,0) T_{[\omega]}+(6,0) T_{\left[\omega^{2}\right]}+(2,1) T_{\left[\omega^{3}\right]}$ & \\
\hline & \multirow[t]{2}{*}{5} & \multirow{2}{*}{$\begin{array}{c}1 \\
\text { local }\end{array}$} & $(\vartheta, 0),(\omega, 0)$ & \multirow[b]{2}{*}{$(17,1)$} \\
\hline & & & $\begin{array}{l}(2,0) U+(1,0) T_{[\vartheta]}+(3,0) T_{[\omega]}+(7,0) T_{\left[\omega^{2}\right]}+ \\
(1,0) T_{\left[\vartheta^{2} \omega^{2}\right]}+(2,1) T_{\left[\omega^{3}\right]}+(1,0) T_{\left[\vartheta^{2} \omega^{4}\right]}\end{array}$ & \\
\hline & \multirow[t]{4}{*}{6} & \multirow{2}{*}{$\begin{array}{c}1 \\
\text { local }\end{array}$} & $(\vartheta, 0),(\omega, 0)$ & \multirow[b]{2}{*}{$(27,3)$} \\
\hline & & & $\begin{array}{l}(2,0) U+(5,2) T_{[\vartheta]}+(3,0) T_{[\omega]}+(9,0) T_{\left[\omega^{2}\right]}+ \\
(3,0) T_{\left[\vartheta^{2} \omega^{2}\right]}+(2,1) T_{\left[\omega^{3}\right]}+(3,0) T_{\left[\vartheta^{2} \omega^{4}\right]}\end{array}$ & \\
\hline & & \multirow{2}{*}{$\begin{array}{c}2 \\
\text { local }\end{array}$} & $\left(\vartheta, \frac{1}{3}\left(2 e_{5}+e_{6}\right)\right),(\omega, 0)$ & \multirow[b]{2}{*}{$(15,3)$} \\
\hline & & & $\begin{array}{l}(2,0) U+(3,0) T_{[\omega]}+(6,0) T_{\left[\omega^{2}\right]}+ \\
(1,1) T_{\left[\vartheta^{2} \omega^{2}\right]}+(2,1) T_{\left[\omega^{3}\right]}+(1,1) T_{\left[\vartheta^{2} \omega^{4}\right]}\end{array}$ & \\
\hline \multirow{6}{*}{$\begin{array}{c}\text { Frobenius } T_{7} \\
\quad[21,1] \\
2935\end{array}$} & \multirow[t]{2}{*}{1} & \multirow{2}{*}{$\begin{array}{c}1 \\
\text { local }\end{array}$} & $(\vartheta, 0),(\omega, 0)$ & \multirow[b]{2}{*}{$(10,2)$} \\
\hline & & & $(1,0) U+(1,1) T_{[\vartheta]}+(7,0) T_{[\omega]}+(1,1) T_{\left[\vartheta^{2}\right]}$ & \\
\hline & \multirow[t]{2}{*}{2} & \multirow{2}{*}{$\begin{array}{c}1 \\
\text { local }\end{array}$} & $(\vartheta, 0),(\omega, 0)$ & \multirow[b]{2}{*}{$(10,2)$} \\
\hline & & & $(1,0) U+(1,1) T_{[\vartheta]}+(7,0) T_{[\omega]}+(1,1) T_{\left[\vartheta^{2}\right]}$ & \\
\hline & \multirow[t]{2}{*}{3} & 1 & $(\vartheta, 0),(\omega, 0)$ & \\
\hline & & local & $(1,0) U+(1,1) T_{[\vartheta]}+(7,0) T_{[\omega]}+(1,1) T_{\left[\vartheta^{2}\right]}$ & $(10,2)$ \\
\hline $\mathbb{Z}_{3} \rtimes \mathbb{Z}_{8}$ & 1 & 1 & $(\vartheta, 0),(\omega, 0)$ & \\
\hline $\begin{array}{l}{[24,1]} \\
6266 \\
\end{array}$ & & local & $\begin{array}{l}(2,0) U+(4,0) T_{[\vartheta]}+(3,2) T_{[\omega]}+(6,0) T_{\left[\vartheta^{2}\right]}+ \\
(4,0) T_{\left[\vartheta^{3}\right]}+(4,0) T_{\left[\vartheta^{2} \omega\right]}+(3,1) T_{\left[\vartheta^{4}\right]} \\
+(1,0) T_{\left[\vartheta^{4} \omega\right]}\end{array}$ & $(27,3)$ \\
\hline $\mathrm{SL}(2,3)-\mathrm{I}$ & 1 & 1 & $(\vartheta, 0),(\omega, 0)$ & \\
\hline$[24,3]$ & & local & $\begin{array}{l}(2,0) U+(3,0) T_{[\vartheta]}+(4,4) T_{[\omega]}+(3,0) T_{\left[\vartheta^{2}\right]}+ \\
(12,0) T_{\left[\vartheta^{2} \omega\right]}+(5,1) T_{\left[\omega^{2}\right]}\end{array}$ & $(29,5)$ \\
\hline 6743 & & 2 & $(\vartheta, 0),\left(\omega, \frac{1}{2} e_{5}\right)$ & \\
\hline & & local & $\begin{array}{l}(2,0) U+(3,0) T_{[\vartheta]}+(3,0) T_{\left[\vartheta^{2}\right]}+ \\
(12,0) T_{\left[\vartheta^{2} \omega\right]}+(5,1) T_{\left[\omega^{2}\right]} \\
(12\end{array}$ & $(25,1)$ \\
\hline & 2 & 1 & $(\vartheta, 0),(\omega, 0)$ & \\
\hline & & local & $\begin{array}{l}(2,0) U+(3,0) T_{[\vartheta]}+(4,4) T_{[\omega]}+(3,0) T_{\left[\vartheta^{2}\right]}+ \\
(12,0) T_{\left[\vartheta^{2} \omega\right]}+(5,1) T_{\left[\omega^{2}\right]}\end{array}$ & $(29,5)$ \\
\hline
\end{tabular}

(Table 2. Continued ...) 


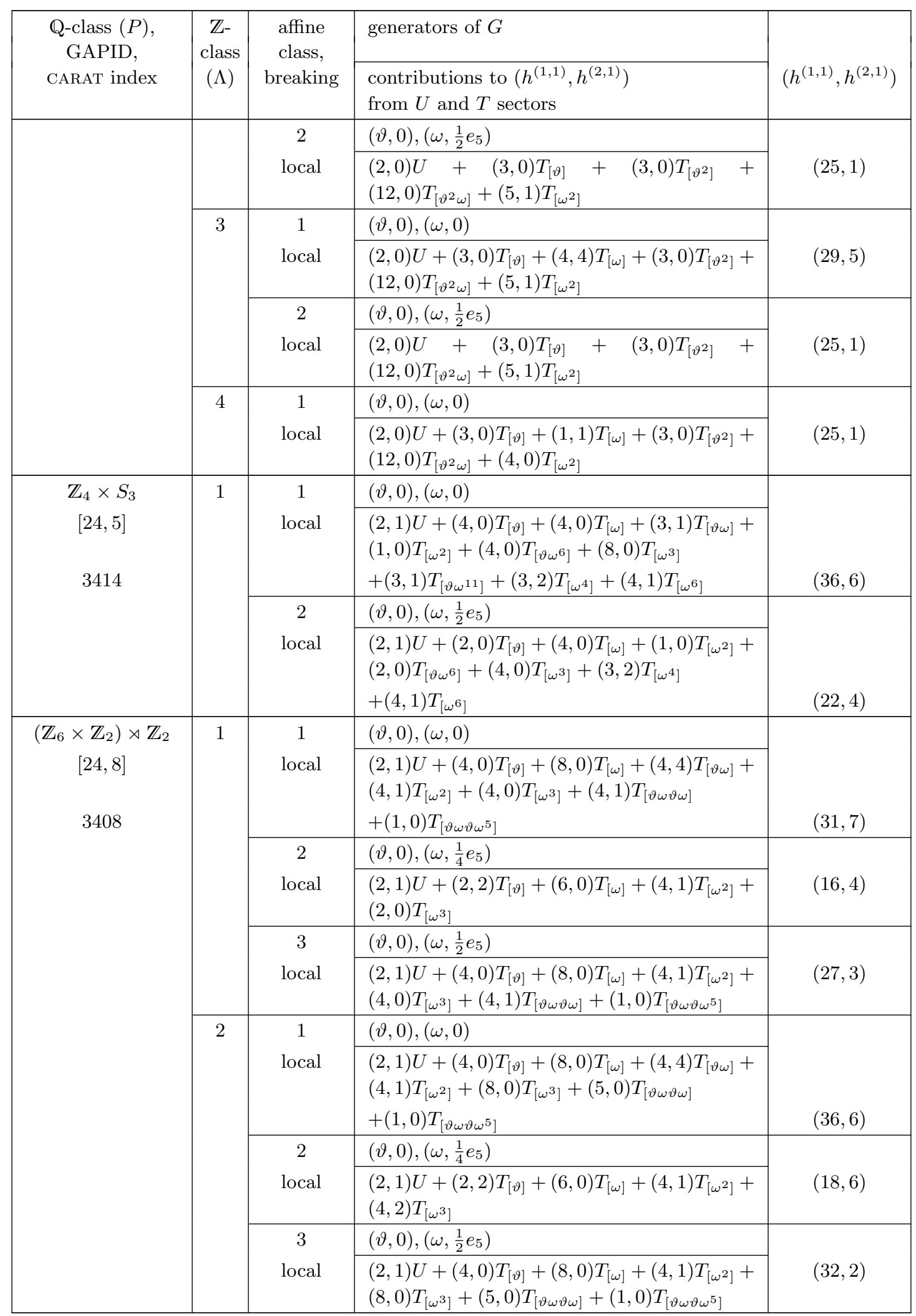

(Table 2. Continued ...) 


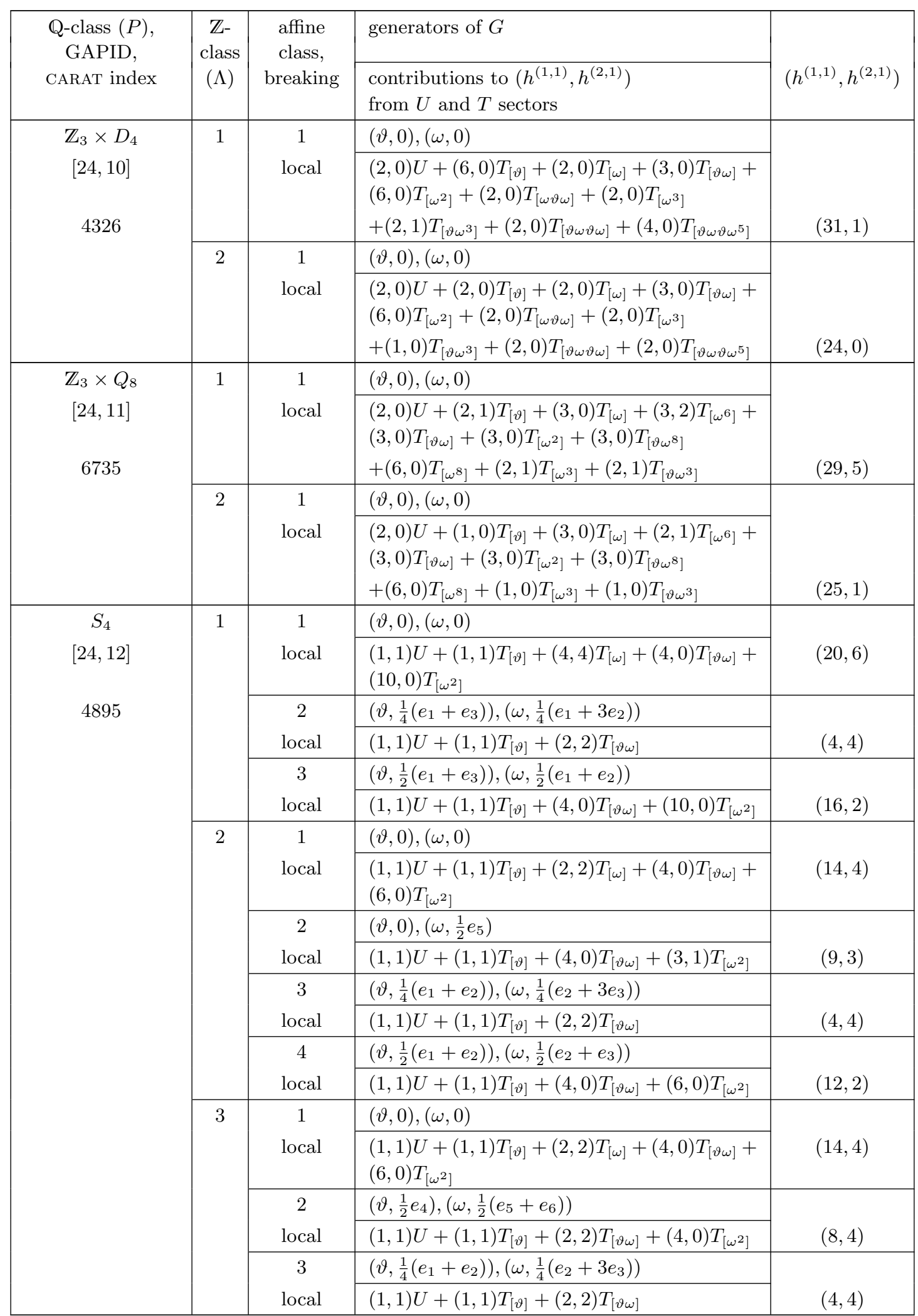

(Table 2. Continued...) 


\begin{tabular}{|c|c|c|c|c|}
\hline \multirow{2}{*}{$\begin{array}{l}\text { Q-class }(P), \\
\text { GAPID, } \\
\text { CARAT index }\end{array}$} & \multirow{2}{*}{$\begin{array}{c}\mathbb{Z}_{-} \\
\text {class } \\
(\Lambda)\end{array}$} & \multirow{2}{*}{$\begin{array}{l}\text { affine } \\
\text { class, } \\
\text { breaking }\end{array}$} & generators of $G$ & \multirow[b]{2}{*}{$\left(h^{(1,1)}, h^{(2,1)}\right)$} \\
\hline & & & $\begin{array}{l}\text { contributions to }\left(h^{(1,1)}, h^{(2,1)}\right) \\
\text { from } U \text { and } T \text { sectors }\end{array}$ & \\
\hline & & \multirow{2}{*}{$\begin{array}{c}4 \\
\text { local }\end{array}$} & $\left(\vartheta, \frac{1}{2}\left(e_{1}+e_{2}\right)\right),\left(\omega, \frac{1}{2}\left(e_{2}+e_{3}\right)\right)$ & \multirow[b]{2}{*}{$(12,2)$} \\
\hline & & & $(1,1) U+(1,1) T_{[\vartheta]}+(4,0) T_{[\vartheta \omega]}+(6,0) T_{\left[\omega^{2}\right]}$ & \\
\hline & \multirow[t]{4}{*}{4} & \multirow{2}{*}{$\begin{array}{c}1 \\
\text { local }\end{array}$} & $(\vartheta, 0),(\omega, 0)$ & \multirow[b]{2}{*}{$(11,3)$} \\
\hline & & & $\begin{array}{l}(1,1) U+(1,1) T_{[\vartheta]}+(1,1) T_{[\omega]}+(4,0) T_{[\vartheta \omega]}+ \\
(4,0) T_{\left[\omega^{2}\right]}\end{array}$ & \\
\hline & & \multirow{2}{*}{$\begin{array}{c}2 \\
\text { local }\end{array}$} & $(\vartheta, 0),\left(\omega, \frac{1}{2} e_{4}\right)$ & \multirow[b]{2}{*}{$(8,2)$} \\
\hline & & & $(1,1) U+(1,1) T_{[\vartheta]}+(4,0) T_{[\vartheta \omega]}+(2,0) T_{\left[\omega^{2}\right]}$ & \\
\hline & \multirow[t]{8}{*}{5} & \multirow{2}{*}{$\begin{array}{c}1 \\
\text { local }\end{array}$} & $(\vartheta, 0),(\omega, 0)$ & \multirow[b]{2}{*}{$(11,3)$} \\
\hline & & & $\begin{array}{l}(1,1) U+(1,1) T_{[\vartheta]}+(1,1) T_{[\omega]}+(4,0) T_{[\vartheta \omega]}+ \\
(4,0) T_{\left[\omega^{2}\right]}\end{array}$ & \\
\hline & & \multirow{2}{*}{$\begin{array}{c}2 \\
\text { local }\end{array}$} & $\left(\vartheta, \frac{1}{2} e_{2}\right),\left(\omega, \frac{1}{2} e_{1}\right)$ & \multirow[b]{2}{*}{$(6,4)$} \\
\hline & & & $(1,1) U+(1,1) T_{[\vartheta]}+(2,2) T_{[\vartheta \omega]}+(2,0) T_{\left[\omega^{2}\right]}$ & \\
\hline & & \multirow{2}{*}{$\begin{array}{c}3 \\
\text { local }\end{array}$} & $\left(\vartheta, \frac{1}{2}\left(e_{4}+e_{5}\right)\right),\left(\omega, \frac{1}{2} e_{4}\right)$ & \multirow[b]{2}{*}{$(8,2)$} \\
\hline & & & $(1,1) U+(1,1) T_{[\vartheta]}+(4,0) T_{[\vartheta \omega]}+(2,0) T_{\left[\omega^{2}\right]}$ & \\
\hline & & \multirow{2}{*}{$\begin{array}{c}4 \\
\text { local }\end{array}$} & $\left(\vartheta, \frac{1}{2}\left(e_{2}+e_{4}+e_{5}\right)\right),\left(\omega, \frac{1}{2}\left(e_{1}+e_{4}\right)\right)$ & \multirow[b]{2}{*}{$(5,5)$} \\
\hline & & & $(1,1) U+(1,1) T_{[\vartheta]}+(2,2) T_{[\vartheta \omega]}+(1,1) T_{\left[\omega^{2}\right]}$ & \\
\hline & \multirow[t]{4}{*}{6} & \multirow{2}{*}{$\begin{array}{c}1 \\
\text { local }\end{array}$} & $(\vartheta, 0),(\omega, 0)$ & \multirow[b]{2}{*}{$(11,3)$} \\
\hline & & & $\begin{array}{l}(1,1) U+(1,1) T_{[\vartheta]}+(1,1) T_{[\omega]}+(4,0) T_{[\vartheta \omega]}+ \\
(4,0) T_{\left[\omega^{2}\right]}\end{array}$ & \\
\hline & & \multirow{2}{*}{$\begin{array}{c}2 \\
\text { local }\end{array}$} & $\left(\vartheta, \frac{1}{2} e_{4}\right),\left(\omega, \frac{1}{2} e_{3}\right)$ & \multirow[b]{2}{*}{$(6,4)$} \\
\hline & & & $(1,1) U+(1,1) T_{[\vartheta]}+(2,2) T_{[\vartheta \omega]}+(2,0) T_{\left[\omega^{2}\right]}$ & \\
\hline \multirow{12}{*}{$\begin{array}{l}\Delta(27) \\
{[27,3]}\end{array}$} & \multirow[t]{8}{*}{1} & \multirow{2}{*}{$\begin{array}{c}1 \\
\text { local }\end{array}$} & $(\vartheta, 0),(\omega, 0)$ & \multirow[b]{2}{*}{$(36,0)$} \\
\hline & & & $\begin{array}{l}(1,0) U+(3,0) T_{[\vartheta]}+(3,0) T_{[\omega]}+(3,0) T_{\left[\vartheta^{2}\right]}+ \\
(3,0) T_{[\vartheta \omega]}+(3,0) T_{\left[\vartheta^{2} \omega\right]}+(3,0) T_{\left[\omega^{2}\right]} \\
+(3,0) T_{[\omega \vartheta \omega]}+(3,0) T_{\left[\omega \vartheta^{2} \omega\right]}+(11,0) T_{\left[\vartheta \omega \vartheta^{2} \omega^{2}\right]}\end{array}$ & \\
\hline & & \multirow{2}{*}{$\begin{array}{c}2 \\
\text { local }\end{array}$} & $\left(\vartheta, \frac{1}{3}\left(e_{2}+e_{5}\right)\right),(\omega, 0)$ & \multirow[b]{2}{*}{$(8,4)$} \\
\hline & & & $\begin{array}{l}(1,0) U+(1,1) T_{[\omega]}+(1,1) T_{[\vartheta \omega]}+(1,1) T_{\left[\omega^{2}\right]}+ \\
(1,1) T_{\left[\omega \vartheta^{2} \omega\right]}+(3,0) T_{\left[\vartheta \omega \vartheta^{2} \omega^{2}\right]}\end{array}$ & \\
\hline & & 3 & $\left(\vartheta, \frac{1}{3}\left(2 e_{1}+2 e_{2}+e_{5}\right)\right),\left(\omega, \frac{1}{3} e_{1}\right)$ & \\
\hline & & non-local & $\begin{array}{l}(1,0) U+(3,0) T_{[\vartheta \omega]}+(3,0) T_{\left[\omega \vartheta^{2} \omega\right]}+ \\
(5,0) T_{\left[\vartheta \omega \vartheta^{2} \omega^{2}\right]}\end{array}$ & $(12,0)$ \\
\hline & & 4 & $\left(\vartheta, \frac{1}{3}\left(2 e_{2}+e_{3}+2 e_{5}\right)\right),\left(\omega, \frac{1}{3} e_{1}\right)$ & \\
\hline & & non-local & $(1,0) U+(3,0) T_{\left[\vartheta \omega \vartheta^{2} \omega^{2}\right]}$ & $(4,0)$ \\
\hline & 2 & 1 & $(\vartheta, 0),(\omega, 0)$ & \\
\hline & & local & $\begin{array}{l}(1,0) U+(1,0) T_{[\vartheta]}+(9,0) T_{[\omega]}+(1,0) T_{\left[\vartheta^{2}\right]}+ \\
(1,0) T_{[\vartheta \omega]}+(1,0) T_{\left[\vartheta^{2} \omega\right]}+(9,0) T_{\left[\omega^{2}\right]} \\
+(1,0) T_{[\omega \vartheta \omega]}+(1,0) T_{\left[\omega \vartheta^{2} \omega\right]}+(11,0) T_{\left[\vartheta \omega \vartheta^{2} \omega^{2}\right]}\end{array}$ & $(36,0)$ \\
\hline & & 2 & $\left(\vartheta, \frac{1}{3}\left(2 e_{3}+e_{4}\right)\right),\left(\omega, \frac{1}{3}\left(e_{1}+e_{4}\right)\right)$ & \\
\hline & & local & $\begin{array}{l}(1,0) U+(1,0) T_{[\vartheta]}+(1,0) T_{\left[\vartheta^{2}\right]}+(1,0) T_{[\vartheta \omega]}+ \\
(1,0) T_{\left[\vartheta^{2} \omega\right]}+(1,0) T_{[\omega \vartheta \omega]}+(1,0) T_{\left[\omega \vartheta^{2} \omega\right]} \\
+(5,0) T_{\left[\vartheta \omega \vartheta^{2} \omega^{2}\right]}\end{array}$ & $(12,0)$ \\
\hline
\end{tabular}

(Table 2. Continued...) 


\begin{tabular}{|c|c|c|c|c|}
\hline \multirow{2}{*}{$\begin{array}{l}\text { Q-class }(P) \\
\text { GAPID, } \\
\text { CARAT index }\end{array}$} & \multirow{2}{*}{$\begin{array}{l}\mathbb{Z}- \\
\text { class } \\
(\Lambda)\end{array}$} & \multirow{2}{*}{$\begin{array}{l}\text { affine } \\
\text { class, } \\
\text { breaking }\end{array}$} & generators of $G$ & \multirow[b]{2}{*}{$\left(h^{(1,1)}, h^{(2,1)}\right)$} \\
\hline & & & $\begin{array}{l}\text { contributions to }\left(h^{(1,1)}, h^{(2,1)}\right) \\
\text { from } U \text { and } T \text { sectors }\end{array}$ & \\
\hline & \multirow[t]{8}{*}{3} & \multirow{2}{*}{$\begin{array}{c}1 \\
\text { local }\end{array}$} & $(\vartheta, 0),(\omega, 0)$ & \multirow[b]{2}{*}{$(36,0)$} \\
\hline & & & $\begin{array}{l}(1,0) U+(3,0) T_{[\vartheta]}+(3,0) T_{[\omega]}+(3,0) T_{\left[\vartheta^{2}\right]}+ \\
(3,0) T_{[\vartheta \omega]}+(3,0) T_{\left[\vartheta^{2} \omega\right]}+(3,0) T_{\left[\omega^{2}\right]} \\
+(3,0) T_{[\omega \vartheta \omega]}+(3,0) T_{\left[\omega \vartheta^{2} \omega\right]}+(11,0) T_{\left[\vartheta \omega \vartheta^{2} \omega^{2}\right]}\end{array}$ & \\
\hline & & \multirow{2}{*}{$\begin{array}{c}2 \\
\text { local }\end{array}$} & $\left(\vartheta, \frac{1}{3}\left(e_{2}+e_{4}+e_{6}\right)\right),(\omega, 0)$ & \multirow[b]{2}{*}{$(20,0)$} \\
\hline & & & $\begin{array}{l}(1,0) U+(3,0) T_{[\vartheta]}+(3,0) T_{[\omega]}+(3,0) T_{\left[\vartheta^{2}\right]}+ \\
(3,0) T_{\left[\omega^{2}\right]}+(7,0) T_{\left[\vartheta \omega \vartheta^{2} \omega^{2}\right]}\end{array}$ & \\
\hline & & \multirow{2}{*}{$\begin{array}{c}3 \\
\text { non-local }\end{array}$} & $\left(\vartheta, \frac{1}{3}\left(2 e_{2}+2 e_{4}+e_{5}+e_{6}\right)\right),(\omega, 0)$ & \multirow[b]{2}{*}{$(12,0)$} \\
\hline & & & $\begin{array}{l}(1,0) U+(3,0) T_{[\omega]}+(3,0) T_{\left[\omega^{2}\right]}+ \\
(5,0) T_{\left[\vartheta \omega \vartheta^{2} \omega^{2}\right]}\end{array}$ & \\
\hline & & \multirow{2}{*}{$\begin{array}{c}4 \\
\text { non-local } \\
\end{array}$} & $\left(\vartheta, \frac{1}{3}\left(e_{2}+e_{4}+e_{5}\right)\right),\left(\omega, \frac{1}{3}\left(e_{1}+e_{3}\right)\right)$ & \multirow[b]{2}{*}{$(4,0)$} \\
\hline & & & $(1,0) U+(3,0) T_{\left[\vartheta \omega \vartheta^{2} \omega^{2}\right]}$ & \\
\hline \multirow{14}{*}{$\begin{array}{c}\left(\mathbb{Z}_{4} \times \mathbb{Z}_{4}\right) \rtimes \mathbb{Z}_{2} \\
{[32,11]}\end{array}$} & \multirow[t]{12}{*}{1} & \multirow{2}{*}{$\begin{array}{c}1 \\
\text { local }\end{array}$} & $(\vartheta, 0),(\omega, 0)$ & \multirow[b]{2}{*}{$(61,1)$} \\
\hline & & & $\begin{array}{l}(2,0) U+(4,0) T_{[\vartheta]}+(4,0) T_{[\omega]}+(9,0) T_{\left[\vartheta^{2}\right]}+ \\
(4,0) T_{[\vartheta \omega]}+(3,1) T_{\left[\vartheta^{2} \omega\right]}+(6,0) T_{\left[\vartheta^{4}\right]} \\
+(12,0) T_{\left[\vartheta^{3} \omega\right]}+(4,0) T_{\left[\vartheta^{7} \omega\right]}+(9,0) T_{[\vartheta \omega \vartheta \omega]}+ \\
(4,0) T_{\left[\vartheta \omega \vartheta^{3} \omega\right]}\end{array}$ & \\
\hline & & \multirow{2}{*}{$\begin{array}{c}2 \\
\text { local }\end{array}$} & $\left(\vartheta, \frac{1}{2} e_{6}\right),\left(\omega, \frac{1}{2} e_{5}\right)$ & \multirow[b]{2}{*}{$(36,0)$} \\
\hline & & & $\begin{array}{l}(2,0) U+(4,0) T_{[\vartheta]}+(2,0) T_{[\omega]}+(3,0) T_{\left[\vartheta^{2}\right]}+ \\
(2,0) T_{[\vartheta \omega]}+(6,0) T_{\left[\vartheta^{4}\right]}+(8,0) T_{\left[\vartheta^{3} \omega\right]} \\
+(2,0) T_{\left[\vartheta^{7} \omega\right]}+(7,0) T_{[\vartheta \omega \vartheta \omega]}\end{array}$ & \\
\hline & & \multirow{2}{*}{$\begin{array}{c}3 \\
\text { local }\end{array}$} & $\left(\vartheta, \frac{1}{2}\left(e_{1}+e_{3}\right)\right),(\omega, 0)$ & \multirow[b]{2}{*}{$(37,1)$} \\
\hline & & & $\begin{array}{l}(2,0) U+(4,0) T_{[\vartheta]}+(4,0) T_{[\omega]}+(7,0) T_{\left[\vartheta^{2}\right]}+ \\
(3,1) T_{\left[\vartheta^{2} \omega\right]}+(4,0) T_{\left[\vartheta^{4}\right]}+(4,0) T_{\left[\vartheta^{3} \omega\right]} \\
+(7,0) T_{[\vartheta \omega \vartheta \omega]}+(2,0) T_{\left[\vartheta \omega \vartheta^{3} \omega\right]}\end{array}$ & \\
\hline & & \multirow{2}{*}{$\begin{array}{c}4 \\
\text { local }\end{array}$} & $\left(\vartheta, \frac{1}{2}\left(e_{1}+e_{3}+e_{6}\right)\right),\left(\omega, \frac{1}{2} e_{5}\right)$ & \multirow[b]{2}{*}{$(24,0)$} \\
\hline & & & $\begin{array}{l}(2,0) U+(4,0) T_{[\vartheta]}+(2,0) T_{[\omega]}+(3,0) T_{\left[\vartheta^{2}\right]}+ \\
(4,0) T_{\left[\vartheta^{4}\right]}+(4,0) T_{\left[\vartheta^{3} \omega\right]}+(5,0) T_{[\vartheta \omega \vartheta \omega]}\end{array}$ & \\
\hline & & \multirow{2}{*}{$\begin{array}{c}5 \\
\text { local }\end{array}$} & $\left(\vartheta, \frac{1}{2} e_{5}\right),(\omega, 0)$ & \multirow[b]{2}{*}{$(54,0)$} \\
\hline & & & $\begin{array}{l}(2,0) U+(4,0) T_{[\vartheta]}+(2,0) T_{[\omega]}+(7,0) T_{\left[\vartheta^{2}\right]}+ \\
(4,0) T_{[\vartheta \omega]}+(6,0) T_{\left[\vartheta^{4}\right]}+(12,0) T_{\left[\vartheta^{3} \omega\right]} \\
+(4,0) T_{\left[\vartheta^{7} \omega\right]}+(9,0) T_{[\vartheta \omega \vartheta \omega]}+(4,0) T_{\left[\vartheta \omega \vartheta^{3} \omega\right]}\end{array}$ & \\
\hline & & \multirow{2}{*}{$\begin{array}{c}6 \\
\text { local }\end{array}$} & $\left(\vartheta, \frac{1}{2}\left(e_{1}+e_{3}+e_{5}\right)\right),(\omega, 0)$ & \multirow[b]{2}{*}{$(30,0)$} \\
\hline & & & $\begin{array}{l}(2,0) U+(4,0) T_{[\vartheta]}+(2,0) T_{[\omega]}+(5,0) T_{\left[\vartheta^{2}\right]}+ \\
(4,0) T_{\left[\vartheta^{4}\right]}+(4,0) T_{\left[\vartheta^{3} \omega\right]}+(7,0) T_{[\vartheta \omega \vartheta \omega]} \\
+(2,0) T_{\left[\vartheta \omega \vartheta^{3} \omega\right]}\end{array}$ & \\
\hline & \multirow[t]{2}{*}{2} & \multirow{2}{*}{$\begin{array}{c}1 \\
\text { local }\end{array}$} & $(\vartheta, 0),(\omega, 0)$ & \multirow[b]{2}{*}{$(42,0)$} \\
\hline & & & $\begin{array}{l}(2,0) U+(4,0) T_{[\vartheta]}+(4,0) T_{[\omega]}+(7,0) T_{\left[\vartheta^{2}\right]}+ \\
(2,0) T_{[\vartheta \omega]}+(2,0) T_{\left[\vartheta^{2} \omega\right]}+(4,0) T_{\left[\vartheta^{4}\right]} \\
+(8,0) T_{\left[\vartheta^{3} \omega\right]}+(2,0) T_{\left[\vartheta^{7} \omega\right]}+(5,0) T_{[\vartheta \omega \vartheta \omega]}+ \\
(2,0) T_{\left[\vartheta \omega \vartheta^{3} \omega\right]}\end{array}$ & \\
\hline
\end{tabular}

(Table 2. Continued ...) 


\begin{tabular}{|c|c|c|c|c|}
\hline \multirow{2}{*}{$\begin{array}{l}\text { Q-class }(P) \\
\text { GAPID, } \\
\text { CARAT index }\end{array}$} & \multirow{2}{*}{$\begin{array}{l}\mathbb{Z}- \\
\text { class } \\
(\Lambda)\end{array}$} & \multirow{2}{*}{$\begin{array}{l}\text { affine } \\
\text { class, } \\
\text { breaking }\end{array}$} & generators of $G$ & \multirow[b]{2}{*}{$\left(h^{(1,1)}, h^{(2,1)}\right)$} \\
\hline & & & $\begin{array}{l}\text { contributions to }\left(h^{(1,1)}, h^{(2,1)}\right) \\
\text { from } U \text { and } T \text { sectors }\end{array}$ & \\
\hline & & \multirow{2}{*}{$\begin{array}{c}2 \\
\text { local }\end{array}$} & $\left(\vartheta, \frac{1}{2} e_{5}\right),(\omega, 0)$ & \multirow[b]{2}{*}{$(37,1)$} \\
\hline & & & $\begin{array}{l}(2,0) U+(4,0) T_{[\vartheta]}+(2,0) T_{[\omega]}+(5,0) T_{\left[\vartheta^{2}\right]}+ \\
(2,0) T_{[\vartheta \omega]}+(1,1) T_{\left[\vartheta^{2} \omega\right]}+(4,0) T_{\left[\vartheta^{4}\right]} \\
+(8,0) T_{\left[\vartheta^{3} \omega\right]}+(2,0) T_{\left[\vartheta^{7} \omega\right]}+(5,0) T_{[\vartheta \omega \vartheta \omega]}+ \\
(2,0) T_{\left[\vartheta \omega \vartheta^{3} \omega\right]}\end{array}$ & \\
\hline & & \multirow{2}{*}{$\begin{array}{c}3 \\
\text { local }\end{array}$} & $\left(\vartheta, \frac{1}{2} e_{2}\right),(\omega, 0)$ & \multirow[b]{2}{*}{$(36,0)$} \\
\hline & & & $\begin{array}{l}(2,0) U+(4,0) T_{[\vartheta]}+(2,0) T_{[\omega]}+(5,0) T_{\left[\vartheta^{2}\right]}+ \\
(2,0) T_{[\vartheta \omega]}+(4,0) T_{\left[\vartheta^{4}\right]}+(8,0) T_{\left[\vartheta^{3} \omega\right]} \\
+(2,0) T_{\left[\vartheta^{7} \omega\right]}+(5,0) T_{[\vartheta \omega \vartheta \omega]}+(2,0) T_{\left[\vartheta \omega \vartheta^{3} \omega\right]}\end{array}$ & \\
\hline & & \multirow{2}{*}{$\begin{array}{c}4 \\
\text { local }\end{array}$} & $\left(\vartheta, \frac{1}{2}\left(e_{1}+e_{3}\right)\right),(\omega, 0)$ & \multirow[b]{2}{*}{$(25,1)$} \\
\hline & & & $\begin{array}{l}(2,0) U+(4,0) T_{[\vartheta]}+(2,0) T_{[\omega]}+(4,0) T_{\left[\vartheta^{2}\right]}+ \\
(1,1) T_{\left[\vartheta^{2} \omega\right]}+(3,0) T_{\left[\vartheta^{4}\right]}+(4,0) T_{\left[\vartheta^{3} \omega\right]} \\
+(4,0) T_{[\vartheta \omega \vartheta \omega]}+(1,0) T_{\left[\vartheta \omega \vartheta^{3} \omega\right]}\end{array}$ & \\
\hline & & \multirow{2}{*}{$\begin{array}{c}5 \\
\text { local }\end{array}$} & $\left(\vartheta, \frac{1}{2}\left(e_{1}+e_{3}+e_{5}\right)\right),(\omega, 0)$ & \multirow[b]{2}{*}{$(30,0)$} \\
\hline & & & $\begin{array}{l}(2,0) U+(4,0) T_{[\vartheta]}+(4,0) T_{[\omega]}+(6,0) T_{\left[\vartheta^{2}\right]}+ \\
(2,0) T_{\left[\vartheta^{2} \omega\right]}+(3,0) T_{\left[\vartheta^{4}\right]}+(4,0) T_{\left[\vartheta^{3} \omega\right]} \\
+(4,0) T_{[\vartheta \omega \vartheta \omega]}+(1,0) T_{\left[\vartheta \omega \vartheta^{3} \omega\right]}\end{array}$ & \\
\hline & & \multirow{2}{*}{$\begin{array}{c}6 \\
\text { local }\end{array}$} & $\left(\vartheta, \frac{1}{2}\left(e_{1}+e_{2}+e_{3}\right)\right),(\omega, 0)$ & \multirow[b]{2}{*}{$(24,0)$} \\
\hline & & & $\begin{array}{l}(2,0) U+(4,0) T_{[\vartheta]}+(2,0) T_{[\omega]}+(4,0) T_{\left[\vartheta^{2}\right]}+ \\
(3,0) T_{\left[\vartheta^{4}\right]}+(4,0) T_{\left[\vartheta^{3} \omega\right]}+(4,0) T_{[\vartheta \omega \vartheta \omega]} \\
+(1,0) T_{\left[\vartheta \omega \vartheta^{3} \omega\right]}\end{array}$ & \\
\hline & \multirow[t]{10}{*}{3} & \multirow{2}{*}{$\begin{array}{c}1 \\
\text { local }\end{array}$} & $(\vartheta, 0),(\omega, 0)$ & \multirow[b]{2}{*}{$(54,0)$} \\
\hline & & & $\begin{array}{l}(2,0) U+(4,0) T_{[\vartheta]}+(8,0) T_{[\omega]}+(10,0) T_{\left[\vartheta^{2}\right]}+ \\
(2,0) T_{[\vartheta \omega]}+(4,0) T_{\left[\vartheta^{2} \omega\right]}+(5,0) T_{\left[\vartheta^{4}\right]} \\
+(8,0) T_{\left[\vartheta^{3} \omega\right]}+(2,0) T_{\left[\vartheta^{7} \omega\right]}+(6,0) T_{[\vartheta \omega \vartheta \omega]}+ \\
(3,0) T_{\left[\vartheta \omega \vartheta^{3} \omega\right]}\end{array}$ & \\
\hline & & \multirow{2}{*}{$\begin{array}{c}2 \\
\text { local }\end{array}$} & $\left(\vartheta, \frac{1}{2} e_{5}\right),(\omega, 0)$ & \multirow[b]{2}{*}{$(42,0)$} \\
\hline & & & $\begin{array}{l}(2,0) U+(4,0) T_{[\vartheta]}+(4,0) T_{[\omega]}+(6,0) T_{\left[\vartheta^{2}\right]}+ \\
(2,0) T_{[\vartheta \omega]}+(5,0) T_{\left[\vartheta^{4}\right]}+(8,0) T_{\left[\vartheta^{3} \omega\right]} \\
+(2,0) T_{\left[\vartheta^{7} \omega\right]}+(6,0) T_{[\vartheta \omega \vartheta \omega]}+(3,0) T_{\left[\vartheta \omega \vartheta^{3} \omega\right]}\end{array}$ & \\
\hline & & \multirow{2}{*}{$\begin{array}{c}3 \\
\text { local }\end{array}$} & $\left(\vartheta, \frac{1}{2} e_{6}\right),\left(\omega, \frac{1}{2} e_{5}\right)$ & \multirow[b]{2}{*}{$(30,0)$} \\
\hline & & & $\begin{array}{l}(2,0) U+(4,0) T_{[\vartheta]}+(4,0) T_{[\omega]}+(3,0) T_{\left[\vartheta^{2}\right]}+ \\
(1,0) T_{[\vartheta \omega]}+(5,0) T_{\left[\vartheta^{4}\right]}+(6,0) T_{\left[\vartheta^{3} \omega\right]} \\
+(1,0) T_{\left[\vartheta^{7} \omega\right]}+(4,0) T_{[\vartheta \omega \vartheta \omega]}\end{array}$ & \\
\hline & & \multirow{2}{*}{$\begin{array}{c}4 \\
\text { local }\end{array}$} & $\left(\vartheta, \frac{1}{2}\left(e_{1}+e_{3}\right)\right),(\omega, 0)$ & \multirow[b]{2}{*}{$(27,3)$} \\
\hline & & & $\begin{array}{l}(2,0) U+(4,0) T_{[\vartheta]}+(4,0) T_{[\omega]}+(3,0) T_{\left[\vartheta^{2}\right]}+ \\
(2,2) T_{\left[\vartheta^{2} \omega\right]}+(4,0) T_{\left[\vartheta^{4}\right]}+(4,0) T_{\left[\vartheta^{3} \omega\right]} \\
+(3,0) T_{[\vartheta \omega \vartheta \omega]}+(1,1) T_{\left[\vartheta \omega \vartheta^{3} \omega\right]}\end{array}$ & \\
\hline & & \multirow{2}{*}{$\begin{array}{c}5 \\
\text { local }\end{array}$} & $\left(\vartheta, \frac{1}{2}\left(e_{1}+e_{3}+e_{5}\right)\right),(\omega, 0)$ & \multirow[b]{2}{*}{$(25,1)$} \\
\hline & & & $\begin{array}{l}(2,0) U+(4,0) T_{[\vartheta]}+(4,0) T_{[\omega]}+(3,0) T_{\left[\vartheta^{2}\right]}+ \\
(4,0) T_{\left[\vartheta^{4}\right]}+(4,0) T_{\left[\vartheta^{3} \omega\right]}+(3,0) T_{[\vartheta \omega \vartheta \omega]} \\
+(1,1) T_{\left[\vartheta \omega \vartheta^{3} \omega\right]}\end{array}$ & \\
\hline
\end{tabular}

(Table 2. Continued ...) 


\begin{tabular}{|c|c|c|c|c|}
\hline \multirow{2}{*}{$\begin{array}{c}\text { Q-class }(P), \\
\text { GAPID, } \\
\text { CARAT index }\end{array}$} & \multirow{2}{*}{$\begin{array}{c}\mathbb{Z}- \\
\text { class } \\
(\Lambda)\end{array}$} & \multirow{2}{*}{$\begin{array}{c}\text { affine } \\
\text { class, } \\
\text { breaking }\end{array}$} & generators of $G$ & \multirow[b]{2}{*}{$\left(h^{(1,1)}, h^{(2,1)}\right)$} \\
\hline & & & $\begin{array}{l}\text { contributions to }\left(h^{(1,1)}, h^{(2,1)}\right) \\
\text { from } U \text { and } T \text { sectors }\end{array}$ & \\
\hline & & \multirow{2}{*}{$\begin{array}{c}6 \\
\text { local }\end{array}$} & $\left(\vartheta, \frac{1}{2}\left(e_{1}+e_{3}+e_{6}\right)\right),\left(\omega, \frac{1}{2} e_{5}\right)$ & \multirow[b]{2}{*}{$(24,0)$} \\
\hline & & & $\begin{array}{l}(2,0) U+(4,0) T_{[\vartheta]}+(4,0) T_{[\omega]}+(3,0) T_{\left[\vartheta^{2}\right]}+ \\
(4,0) T_{\left[\vartheta^{4}\right]}+(4,0) T_{\left[\vartheta^{3} \omega\right]}+(3,0) T_{[\vartheta \omega \vartheta \omega]}\end{array}$ & \\
\hline & & 7 & $\left(\vartheta, \frac{1}{2} e_{4}\right),\left(\omega, \frac{1}{2}\left(e_{1}+e_{2}\right)\right)$ & \multirow[b]{2}{*}{$(39,3)$} \\
\hline & & local & $\begin{array}{l}(2,0) U+(4,0) T_{[\vartheta]}+(6,0) T_{\left[\vartheta^{2}\right]}+(2,0) T_{[\vartheta \omega]}+ \\
(2,2) T_{\left[\vartheta^{2} \omega\right]}+(4,1) T_{\left[\vartheta^{4}\right]}+(8,0) T_{\left[\vartheta^{3} \omega\right]} \\
+(2,0) T_{\left[\vartheta^{7} \omega\right]}+(6,0) T_{[\vartheta \omega \vartheta \omega]}+(3,0) T_{\left[\vartheta \omega \vartheta^{3} \omega\right]}\end{array}$ & \\
\hline & & 8 & $\left(\vartheta, \frac{1}{2}\left(e_{4}+e_{5}\right)\right),\left(\omega, \frac{1}{2}\left(e_{1}+e_{2}\right)\right)$ & \multirow[b]{2}{*}{$(37,1)$} \\
\hline & & local & $\begin{array}{l}(2,0) U+(4,0) T_{[\vartheta]}+(6,0) T_{\left[\vartheta^{2}\right]}+(2,0) T_{[\vartheta \omega]}+ \\
(4,1) T_{\left[\vartheta^{4}\right]}+(8,0) T_{\left[\vartheta^{3} \omega\right]}+(2,0) T_{\left[\vartheta^{7} \omega\right]} \\
+(6,0) T_{[\vartheta \omega \vartheta \omega]}+(3,0) T_{\left[\vartheta \omega \vartheta^{3} \omega\right]}\end{array}$ & \\
\hline & & \multirow{2}{*}{$\begin{array}{c}9 \\
\text { local }\end{array}$} & $\left(\vartheta, \frac{1}{2}\left(e_{4}+e_{6}\right)\right),\left(\omega, \frac{1}{2}\left(e_{1}+e_{2}+e_{5}\right)\right)$ & \multirow[b]{2}{*}{$(25,1)$} \\
\hline & & & $\begin{array}{l}(2,0) U+(4,0) T_{[\vartheta]}+(3,0) T_{\left[\vartheta^{2}\right]}+(1,0) T_{[\vartheta \omega]}+ \\
(4,1) T_{\left[\vartheta^{4}\right]}+(6,0) T_{\left[\vartheta^{3} \omega\right]}+(1,0) T_{\left[\vartheta^{7} \omega\right]} \\
+(4,0) T_{[\vartheta \omega \vartheta \omega]}\end{array}$ & \\
\hline & & \multirow{2}{*}{$\begin{array}{c}10 \\
\text { local }\end{array}$} & $\left(\vartheta, \frac{1}{2}\left(e_{1}+e_{3}+e_{4}\right)\right),\left(\omega, \frac{1}{2}\left(e_{1}+e_{2}\right)\right)$ & \multirow[b]{2}{*}{$(22,4)$} \\
\hline & & & $\begin{array}{l}(2,0) U+(4,0) T_{[\vartheta]}+(3,0) T_{\left[\vartheta^{2}\right]}+(2,2) T_{\left[\vartheta^{2} \omega\right]}+ \\
(3,1) T_{\left[\vartheta^{4}\right]}+(4,0) T_{\left[\vartheta^{3} \omega\right]}+(3,0) T_{[\vartheta \omega \vartheta \omega]} \\
+(1,1) T_{\left[\vartheta \omega \vartheta^{3} \omega\right]}\end{array}$ & \\
\hline & & \multirow{2}{*}{$\begin{array}{c}11 \\
\text { local }\end{array}$} & $\left(\vartheta, \frac{1}{2}\left(e_{1}+e_{3}+e_{4}+e_{5}\right)\right),\left(\omega, \frac{1}{2}\left(e_{1}+e_{2}\right)\right)$ & \multirow[b]{2}{*}{$(20,2)$} \\
\hline & & & $\begin{array}{l}(2,0) U+(4,0) T_{[\vartheta]}+(3,0) T_{\left[\vartheta^{2}\right]}+(3,1) T_{\left[\vartheta^{4}\right]}+ \\
(4,0) T_{\left[\vartheta^{3} \omega\right]}+(3,0) T_{[\vartheta \omega \vartheta \omega]}+(1,1) T_{\left[\vartheta \omega \vartheta^{3} \omega\right]}\end{array}$ & \\
\hline & & \multirow{2}{*}{$\begin{array}{c}12 \\
\text { local }\end{array}$} & $\left(\vartheta, \frac{1}{2}\left(e_{1}+e_{3}+e_{4}+e_{6}\right)\right),\left(\omega, \frac{1}{2}\left(e_{1}+e_{2}+e_{5}\right)\right)$ & \multirow[b]{2}{*}{$(19,1)$} \\
\hline & & & $\begin{array}{l}(2,0) U+(4,0) T_{[\vartheta]}+(3,0) T_{\left[\vartheta^{2}\right]}+(3,1) T_{\left[\vartheta^{4}\right]}+ \\
(4,0) T_{\left[\vartheta^{3} \omega\right]}+(3,0) T_{[\vartheta \omega \vartheta \omega]}\end{array}$ & \\
\hline & \multirow[t]{6}{*}{4} & \multirow{2}{*}{$\begin{array}{c}1 \\
\text { local }\end{array}$} & $(\vartheta, 0),(\omega, 0)$ & \multirow[b]{2}{*}{$(42,0)$} \\
\hline & & & $\begin{array}{l}(2,0) U+(4,0) T_{[\vartheta]}+(4,0) T_{[\omega]}+(7,0) T_{\left[\vartheta^{2}\right]}+ \\
(2,0) T_{[\vartheta \omega]}+(2,0) T_{\left[\vartheta^{2} \omega\right]}+(4,0) T_{\left[\vartheta^{4}\right]} \\
+(8,0) T_{\left[\vartheta^{3} \omega\right]}+(2,0) T_{\left[\vartheta^{7} \omega\right]}+(5,0) T_{[\vartheta \omega \vartheta \omega]}+ \\
(2,0) T_{\left[\vartheta \omega \vartheta^{3} \omega\right]}\end{array}$ & \\
\hline & & \multirow{2}{*}{$\begin{array}{c}2 \\
\text { local }\end{array}$} & $\left(\vartheta, \frac{1}{2}\left(e_{2}+e_{3}+e_{4}+e_{5}+e_{6}\right)\right),\left(\omega, \frac{1}{2}\left(e_{1}+e_{2}+e_{4}\right)\right)$ & \multirow[b]{2}{*}{$(19,1)$} \\
\hline & & & $\begin{array}{l}(2,0) U+(4,0) T_{[\vartheta]}+(2,0) T_{[\omega]}+(3,0) T_{\left[\vartheta^{2}\right]}+ \\
(2,0) T_{\left[\vartheta^{4}\right]}+(4,0) T_{\left[\vartheta^{3} \omega\right]}+(2,1) T_{[\vartheta \omega \vartheta \omega]}\end{array}$ & \\
\hline & & \multirow{2}{*}{$\begin{array}{c}3 \\
\text { local }\end{array}$} & $\left(\vartheta, \frac{1}{2} e_{3}\right),\left(\omega, \frac{1}{2} e_{2}\right)$ & \multirow[b]{2}{*}{$(30,0)$} \\
\hline & & & $\begin{array}{l}(2,0) U+(4,0) T_{[\vartheta]}+(2,0) T_{[\omega]}+(4,0) T_{\left[\vartheta^{2}\right]}+ \\
(1,0) T_{[\vartheta \omega]}+(4,0) T_{\left[\vartheta^{4}\right]}+(6,0) T_{\left[\vartheta^{3} \omega\right]} \\
+(1,0) T_{\left[\vartheta^{7} \omega\right]}+(5,0) T_{[\vartheta \omega \vartheta \omega]}+(1,0) T_{\left[\vartheta \omega \vartheta^{3} \omega\right]}\end{array}$ & \\
\hline & \multirow[t]{2}{*}{5} & \multirow{2}{*}{$\begin{array}{c}1 \\
\text { local }\end{array}$} & $(\vartheta, 0),(\omega, 0)$ & \multirow[b]{2}{*}{$(30,0)$} \\
\hline & & & $\begin{array}{l}(2,0) U+(4,0) T_{[\vartheta]}+(3,0) T_{[\omega]}+(5,0) T_{\left[\vartheta^{2}\right]}+ \\
(1,0) T_{[\vartheta \omega]}+(1,0) T_{\left[\vartheta^{2} \omega\right]}+(3,0) T_{\left[\vartheta^{4}\right]} \\
+(6,0) T_{\left[\vartheta^{3} \omega\right]}+(1,0) T_{\left[\vartheta^{7} \omega\right]}+(3,0) T_{[\vartheta \omega \vartheta \omega]}+ \\
(1,0) T_{\left[\vartheta \omega \vartheta^{3} \omega\right]}\end{array}$ & \\
\hline
\end{tabular}

(Table 2. Continued ...) 


\begin{tabular}{|c|c|c|c|c|}
\hline \multirow{6}{*}{$\begin{array}{l}\text { Q-class }(P) \\
\text { GAPID, } \\
\text { CARAT index }\end{array}$} & \multirow{6}{*}{$\begin{array}{c}\mathbb{Z}- \\
\text { class } \\
(\Lambda)\end{array}$} & \multirow{2}{*}{$\begin{array}{l}\text { affine } \\
\text { class, } \\
\text { breaking }\end{array}$} & generators of $G$ & \multirow[b]{2}{*}{$\left(h^{(1,1)}, h^{(2,1)}\right)$} \\
\hline & & & $\begin{array}{l}\text { contributions to }\left(h^{(1,1)}, h^{(2,1)}\right) \\
\text { from } U \text { and } T \text { sectors }\end{array}$ & \\
\hline & & \multirow{2}{*}{$\begin{array}{c}2 \\
\text { local }\end{array}$} & $\left(\vartheta, \frac{1}{2}\left(e_{1}+e_{2}\right)+\frac{1}{4}\left(e_{3}+3 e_{4}\right)\right),(\omega, 0)$ & \multirow[b]{2}{*}{$(16,1)$} \\
\hline & & & $\begin{array}{l}(2,0) U+(4,0) T_{[\vartheta]}+(1,1) T_{[\omega]}+(3,0) T_{\left[\vartheta^{2}\right]}+ \\
(1,0) T_{\left[\vartheta^{4}\right]}+(4,0) T_{\left[\vartheta^{3} \omega\right]}+(1,0) T_{[\vartheta \omega \vartheta \omega]}\end{array}$ & \\
\hline & & \multirow{2}{*}{$\begin{array}{c}3 \\
\text { local }\end{array}$} & $\left(\vartheta, \frac{1}{2}\left(e_{3}+e_{4}\right)\right),(\omega, 0)$ & \\
\hline & & & $\begin{array}{l}(2,0) U+(4,0) T_{[\vartheta]}+(3,0) T_{[\omega]}+(5,0) T_{\left[\vartheta^{2}\right]}+ \\
(1,0) T_{[\vartheta \omega]}+(1,0) T_{\left[\vartheta^{2} \omega\right]}+(3,0) T_{\left[\vartheta^{4}\right]} \\
+(6,0) T_{\left[\vartheta^{3} \omega\right]}+(1,0) T_{\left[\vartheta^{7} \omega\right]}+(3,0) T_{[\vartheta \omega \vartheta \omega]}+ \\
(1,0) T_{\left[\vartheta \omega \vartheta^{3} \omega\right]}\end{array}$ & $(30,0)$ \\
\hline \multirow{2}{*}{$\begin{array}{c}\mathbb{Z}_{3} \times\left(\mathbb{Z}_{3} \rtimes \mathbb{Z}_{4}\right) \\
{[36,6]}\end{array}$} & \multirow[t]{2}{*}{1} & \multirow{2}{*}{$\begin{array}{c}1 \\
\text { local }\end{array}$} & $(\vartheta, 0),(\omega, 0)$ & \multirow[b]{2}{*}{$(51,3)$} \\
\hline & & & $\begin{array}{l}(2,0) U+(5,0) T_{[\vartheta]}+(3,0) T_{[\omega]}+(3,0) T_{\left[\omega^{2}\right]}+ \\
(6,0) T_{\left[\vartheta^{2} \omega^{2}\right]}+(2,1) T_{\left[\omega^{3}\right]}+(9,0) T_{\left[\omega^{4}\right]} \\
+(6,0) T_{\left[\vartheta^{2} \omega^{4}\right]}+(3,0) T_{\left[\omega^{5}\right]}+(3,1) T_{\left[\omega^{6}\right]}+ \\
(1,0) T_{\left[\vartheta^{2} \omega^{6}\right]}+(6,0) T_{\left[\vartheta^{2} \omega^{8}\right]}+(2,1) T_{\left[\omega^{9}\right]}\end{array}$ & \\
\hline \multirow{6}{*}{$\begin{array}{c}\mathbb{Z}_{3} \times A_{4} \\
{[36,11]}\end{array}$} & \multirow[t]{2}{*}{1} & \multirow{2}{*}{$\begin{array}{c}1 \\
\text { local }\end{array}$} & $(\vartheta, 0),(\omega, 0)$ & \multirow[b]{2}{*}{$(20,0)$} \\
\hline & & & $\begin{array}{l}(1,0) U+(1,0) T_{[\vartheta]}+(2,0) T_{[\omega]}+(1,0) T_{\left[\vartheta^{2}\right]}+ \\
(1,0) T_{[\vartheta \omega]}+(1,0) T_{\left[\vartheta^{2} \omega\right]}+(5,0) T_{\left[\omega^{2}\right]} \\
+(1,0) T_{[\omega \vartheta \omega]}+(1,0) T_{\left[\omega \vartheta^{2} \omega\right]}+(6,0) T_{\left[\omega^{3}\right]}\end{array}$ & \\
\hline & \multirow[t]{2}{*}{2} & \multirow{2}{*}{$\begin{array}{c}1 \\
\text { local }\end{array}$} & $(\vartheta, 0),(\omega, 0)$ & \multirow[b]{2}{*}{$(16,0)$} \\
\hline & & & $\begin{array}{l}(1,0) U+(1,0) T_{[\vartheta]}+(2,0) T_{[\omega]}+(1,0) T_{\left[\vartheta^{2}\right]}+ \\
(1,0) T_{[\vartheta \omega]}+(1,0) T_{\left[\vartheta^{2} \omega\right]}+(5,0) T_{\left[\omega^{2}\right]} \\
+(1,0) T_{[\omega \vartheta \omega]}+(1,0) T_{\left[\omega \vartheta^{2} \omega\right]}+(2,0) T_{\left[\omega^{3}\right]}\end{array}$ & \\
\hline & \multirow[t]{2}{*}{3} & \multirow{2}{*}{$\begin{array}{c}1 \\
\text { local }\end{array}$} & $(\vartheta, 0),(\omega, 0)$ & \multirow[b]{2}{*}{$(16,0)$} \\
\hline & & & $\begin{array}{l}(1,0) U+(1,0) T_{[\vartheta]}+(2,0) T_{[\omega]}+(1,0) T_{\left[\vartheta^{2}\right]}+ \\
(1,0) T_{[\vartheta \omega]}+(1,0) T_{\left[\vartheta^{2} \omega\right]}+(5,0) T_{\left[\omega^{2}\right]} \\
+(1,0) T_{[\omega \vartheta \omega]}+(1,0) T_{\left[\omega \vartheta^{2} \omega\right]}+(2,0) T_{\left[\omega^{3}\right]} \\
\end{array}$ & \\
\hline \multirow{6}{*}{$\begin{array}{c}\mathbb{Z}_{6} \times S_{3} \\
{[36,12]}\end{array}$} & \multirow[t]{4}{*}{1} & \multirow{2}{*}{$\begin{array}{c}1 \\
\text { local }\end{array}$} & $(\vartheta, 0),(\omega, 0)$ & \multirow[b]{2}{*}{$(48,0)$} \\
\hline & & & $\begin{array}{l}(2,0) U+(2,0) T_{[\vartheta]}+(1,0) T_{[\omega]}+(9,0) T_{\left[\vartheta^{2}\right]}+ \\
(2,0) T_{[\vartheta \omega]}+(2,0) T_{\left[\vartheta^{3}\right]}+(6,0) T_{\left[\vartheta^{2} \omega\right]} \\
+(2,0) T_{\left[\vartheta^{3} \omega\right]}+(5,0) T_{\left[\omega^{2}\right]}+(6,0) T_{\left[\vartheta^{2} \omega^{2}\right]}+ \\
(6,0) T_{\left[\vartheta^{4} \omega^{2}\right]}+(3,0) T_{\left[\omega^{3}\right]}+(2,0) T_{\left[\vartheta^{2} \omega^{3}\right]}\end{array}$ & \\
\hline & & \multirow{2}{*}{$\begin{array}{c}2 \\
\text { local }\end{array}$} & $(\vartheta, 0),\left(\omega, \frac{1}{3}\left(e_{5}+e_{6}\right)\right)$ & \\
\hline & & & $\begin{array}{l}(2,0) U+(2,0) T_{[\vartheta]}+(4,0) T_{\left[\vartheta^{2}\right]}+(2,0) T_{[\vartheta \omega]}+ \\
(2,0) T_{\left[\vartheta^{3}\right]}+(4,0) T_{\left[\vartheta^{2} \omega\right]}+(2,0) T_{\left[\vartheta^{3} \omega\right]} \\
+(2,1) T_{\left[\vartheta^{2} \omega^{2}\right]}+(2,1) T_{\left[\vartheta^{4} \omega^{2}\right]}+(3,0) T_{\left[\omega^{3}\right]}+ \\
(1,0) T_{\left[\vartheta^{2} \omega^{3}\right]}\end{array}$ & $(26,2)$ \\
\hline & \multirow[t]{2}{*}{2} & \multirow{2}{*}{$\begin{array}{c}1 \\
\text { local }\end{array}$} & $(\vartheta, 0),(\omega, 0)$ & \\
\hline & & & $\begin{array}{l}(2,0) U+(2,0) T_{[\vartheta]}+(1,0) T_{[\omega]}+(6,0) T_{\left[\vartheta^{2}\right]}+ \\
(2,0) T_{[\vartheta \omega]}+(2,0) T_{\left[\vartheta^{3}\right]}+(6,0) T_{\left[\vartheta^{2} \omega\right]} \\
+(2,0) T_{\left[\vartheta^{3} \omega\right]}+(3,1) T_{\left[\omega^{2}\right]}+(3,0) T_{\left[\vartheta^{2} \omega^{2}\right]}+ \\
(3,0) T_{\left[\vartheta^{4} \omega^{2}\right]}+(3,0) T_{\left[\omega^{3}\right]}+(2,0) T_{\left[\vartheta^{2} \omega^{3}\right]}\end{array}$ & $(37,1)$ \\
\hline
\end{tabular}

(Table 2. Continued ...) 


\begin{tabular}{|c|c|c|c|c|}
\hline \multirow{4}{*}{$\begin{array}{l}\text { Q-class }(P), \\
\text { GAPID, } \\
\text { CARAT index }\end{array}$} & \multirow{4}{*}{$\begin{array}{c}\mathbb{Z}- \\
\text { class } \\
(\Lambda)\end{array}$} & \multirow{2}{*}{$\begin{array}{c}\text { affine } \\
\text { class, } \\
\text { breaking }\end{array}$} & generators of $G$ & \multirow[b]{2}{*}{$\left(h^{(1,1)}, h^{(2,1)}\right)$} \\
\hline & & & $\begin{array}{l}\text { contributions to }\left(h^{(1,1)}, h^{(2,1)}\right) \\
\text { from } U \text { and } T \text { sectors }\end{array}$ & \\
\hline & & \multirow{2}{*}{$\begin{array}{c}2 \\
\text { local }\end{array}$} & $(\vartheta, 0),\left(\omega, \frac{1}{3}\left(2 e_{5}+e_{6}\right)\right)$ & \multirow[b]{2}{*}{$(24,0)$} \\
\hline & & & $\begin{array}{l}(2,0) U+(2,0) T_{[\vartheta]}+(4,0) T_{\left[\vartheta^{2}\right]}+(2,0) T_{[\vartheta \omega]}+ \\
(2,0) T_{\left[\vartheta^{3}\right]}+(4,0) T_{\left[\vartheta^{2} \omega\right]}+(2,0) T_{\left[\vartheta^{3} \omega\right]} \\
+(1,0) T_{\left[\vartheta^{2} \omega^{2}\right]}+(1,0) T_{\left[\vartheta^{4} \omega^{2}\right]}+(3,0) T_{\left[\omega^{3}\right]}+ \\
(1,0) T_{\left[\vartheta^{2} \omega^{3}\right]}\end{array}$ & \\
\hline$\Delta(48)$ & \multirow[t]{4}{*}{1} & \multirow{2}{*}{$\begin{array}{c}1 \\
\text { local }\end{array}$} & $(\vartheta, 0),(\omega, 0)$ & \multirow[b]{2}{*}{$(32,2)$} \\
\hline$[48,3]$ & & & $\begin{array}{l}(1,0) U+(1,1) T_{[\vartheta]}+(4,0) T_{[\omega]}+(1,1) T_{\left[\vartheta^{2}\right]}+ \\
(9,0) T_{\left[\omega^{2}\right]}+(4,0) T_{\left[\omega^{3}\right]}+(12,0) T_{\left[\vartheta \omega^{2} \vartheta^{2} \omega^{3}\right]}\end{array}$ & \\
\hline \multirow[t]{14}{*}{2774} & & \multirow{2}{*}{$\begin{array}{c}2 \\
\text { local }\end{array}$} & $\left(\vartheta, \frac{1}{2}\left(e_{1}+e_{2}\right)\right),\left(\omega, \frac{1}{2}\left(e_{1}+e_{3}\right)\right)$ & \multirow[b]{2}{*}{$(12,2)$} \\
\hline & & & $\begin{array}{l}(1,0) U+(1,1) T_{[\vartheta]}+(1,1) T_{\left[\vartheta^{2}\right]}+(5,0) T_{\left[\omega^{2}\right]}+ \\
(4,0) T_{\left[\vartheta \omega^{2} \vartheta^{2} \omega^{3}\right]}\end{array}$ & \\
\hline & \multirow[t]{6}{*}{2} & \multirow{2}{*}{$\begin{array}{c}1 \\
\text { local }\end{array}$} & $(\vartheta, 0),(\omega, 0)$ & \multirow[b]{2}{*}{$(20,2)$} \\
\hline & & & $\begin{array}{l}(1,0) U+(1,1) T_{[\vartheta]}+(2,0) T_{[\omega]}+(1,1) T_{\left[\vartheta^{2}\right]}+ \\
(5,0) T_{\left[\omega^{2}\right]}+(2,0) T_{\left[\omega^{3}\right]}+(8,0) T_{\left[\vartheta \omega^{2} \vartheta^{2} \omega^{3}\right]}\end{array}$ & \\
\hline & & \multirow{2}{*}{$\begin{array}{c}2 \\
\text { local }\end{array}$} & $(\vartheta, 0),\left(\omega, \frac{1}{2}\left(e_{1}+e_{3}\right)\right)$ & \multirow[b]{2}{*}{$(9,3)$} \\
\hline & & & $\begin{array}{l}(1,0) U+(1,1) T_{[\vartheta]}+(1,1) T_{\left[\vartheta^{2}\right]}+(2,1) T_{\left[\omega^{2}\right]}+ \\
(4,0) T_{\left[\vartheta \omega^{2} \vartheta^{2} \omega^{3}\right]}\end{array}$ & \\
\hline & & \multirow{2}{*}{$\begin{array}{c}3 \\
\text { local }\end{array}$} & $\left(\vartheta, \frac{1}{2}\left(e_{1}+e_{3}\right)\right),(\omega, 0)$ & \multirow[b]{2}{*}{$(16,2)$} \\
\hline & & & $\begin{array}{l}(1,0) U+(1,1) T_{[\vartheta]}+(1,0) T_{[\omega]}+(1,1) T_{\left[\vartheta^{2}\right]}+ \\
(5,0) T_{\left[\omega^{2}\right]}+(1,0) T_{\left[\omega^{3}\right]}+(6,0) T_{\left[\vartheta \omega^{2} \vartheta^{2} \omega^{3}\right]} \\
\end{array}$ & \\
\hline & \multirow[t]{2}{*}{3} & \multirow{2}{*}{$\begin{array}{c}1 \\
\text { local }\end{array}$} & $(\vartheta, 0),(\omega, 0)$ & \multirow[b]{2}{*}{$(20,2)$} \\
\hline & & & $\begin{array}{l}(1,0) U+(1,1) T_{[\vartheta]}+(2,0) T_{[\omega]}+(1,1) T_{\left[\vartheta^{2}\right]}+ \\
(5,0) T_{\left[\omega^{2}\right]}+(2,0) T_{\left[\omega^{3}\right]}+(8,0) T_{\left[\vartheta \omega^{2} \vartheta^{2} \omega^{3}\right]}\end{array}$ & \\
\hline & \multirow[t]{4}{*}{4} & \multirow{2}{*}{$\begin{array}{c}1 \\
\text { local }\end{array}$} & $(\vartheta, 0),(\omega, 0)$ & \multirow[b]{2}{*}{$(14,2)$} \\
\hline & & & $\begin{array}{l}(1,0) U+(1,1) T_{[\vartheta]}+(1,0) T_{[\omega]}+(1,1) T_{\left[\vartheta^{2}\right]}+ \\
(3,0) T_{\left[\omega^{2}\right]}+(1,0) T_{\left[\omega^{3}\right]}+(6,0) T_{\left[\vartheta \omega^{2} \vartheta^{2} \omega^{3}\right]}\end{array}$ & \\
\hline & & \multirow{2}{*}{$\begin{array}{c}2 \\
\text { local }\end{array}$} & $\left(\vartheta, \frac{1}{2}\left(e_{1}+e_{3}\right)\right),\left(\omega, \frac{1}{2}\left(e_{1}+e_{2}\right)\right)$ & \multirow[b]{2}{*}{$(8,2)$} \\
\hline & & & $\begin{array}{l}(1,0) U+(1,1) T_{[\vartheta]}+(1,1) T_{\left[\vartheta^{2}\right]}+(1,0) T_{\left[\omega^{2}\right]}+ \\
(4,0) T_{\left[\vartheta \omega^{2} \vartheta^{2} \omega^{3}\right]}\end{array}$ & \\
\hline $\mathrm{GL}(2,3)$ & 1 & 1 & $(\vartheta, 0),(\omega, 0)$ & \multirow[b]{2}{*}{$(26,8)$} \\
\hline$[48,29]$ & & local & $\begin{array}{l}(2,1) U+(5,5) T_{[\vartheta]}+(8,0) T_{[\omega]}+(4,0) T_{[\vartheta \omega]}+ \\
(3,1) T_{\left[\omega^{2}\right]}+(1,1) T_{\left[\omega \vartheta^{2} \omega\right]}+(3,0) T_{\left[\omega^{4}\right]}\end{array}$ & \\
\hline \multirow[t]{6}{*}{5713} & & \multirow{2}{*}{$\begin{array}{c}2 \\
\text { local }\end{array}$} & $\left(\vartheta, \frac{1}{3} e_{6}\right),\left(\omega, \frac{1}{3} e_{6}\right)$ & \multirow[b]{2}{*}{$(20,2)$} \\
\hline & & & $\begin{array}{l}(2,1) U+(8,0) T_{[\omega]}+(4,0) T_{[\vartheta \omega]}+(3,1) T_{\left[\omega^{2}\right]}+ \\
(3,0) T_{\left[\omega^{4}\right]}\end{array}$ & \\
\hline & & \multirow{2}{*}{$\begin{array}{c}3 \\
\text { local }\end{array}$} & $\left(\vartheta, \frac{1}{2} e_{2}+\frac{2}{3} e_{5}\right),\left(\omega, \frac{1}{2} e_{2}+\frac{2}{3} e_{5}\right)$ & \\
\hline & & & $\begin{array}{l}(2,1) U+(8,0) T_{[\omega]}+(4,0) T_{[\vartheta \omega]}+(3,1) T_{\left[\omega^{2}\right]}+ \\
(3,0) T_{\left[\omega^{4}\right]}\end{array}$ & $(20,2)$ \\
\hline & & 4 & $\left(\vartheta, \frac{1}{2} e_{2}\right),\left(\omega, \frac{1}{2} e_{2}\right)$ & \\
\hline & & local & $\begin{array}{l}(2,1) U+(5,5) T_{[\vartheta]}+(8,0) T_{[\omega]}+(4,0) T_{[\vartheta \omega]}+ \\
(3,1) T_{\left[\omega^{2}\right]}+(1,1) T_{\left[\omega \vartheta^{2} \omega\right]}+(3,0) T_{\left[\omega^{4}\right]}\end{array}$ & $(26,8)$ \\
\hline
\end{tabular}




\begin{tabular}{|c|c|c|c|c|}
\hline \multirow{2}{*}{$\begin{array}{l}\text { Q-class }(P), \\
\text { GAPID, } \\
\text { CARAT index }\end{array}$} & \multirow{2}{*}{$\begin{array}{c}\mathbb{Z}- \\
\text { class } \\
(\Lambda)\end{array}$} & \multirow{2}{*}{$\begin{array}{c}\text { affine } \\
\text { class, } \\
\text { breaking }\end{array}$} & generators of $G$ & \multirow[b]{2}{*}{$\left(h^{(1,1)}, h^{(2,1)}\right)$} \\
\hline & & & $\begin{array}{l}\text { contributions to }\left(h^{(1,1)}, h^{(2,1)}\right) \\
\text { from } U \text { and } T \text { sectors }\end{array}$ & \\
\hline \multirow{6}{*}{$\begin{array}{c}\mathrm{SL}(2,3) \rtimes \mathbb{Z}_{2} \\
{[48,33]}\end{array}$} & \multirow[t]{6}{*}{1} & \multirow{2}{*}{$\begin{array}{c}1 \\
\text { local }\end{array}$} & $(\vartheta, 0),(\omega, 0)$ & \multirow[b]{2}{*}{$(41,5)$} \\
\hline & & & $\begin{array}{l}(2,1) U+(4,0) T_{[\vartheta]}+(4,0) T_{[\omega]}+(3,0) T_{\left[\omega^{6}\right]}+ \\
(4,0) T_{\left[\omega^{7}\right]}+(1,0) T_{\left[\omega^{2}\right]}+(3,2) T_{[\omega \vartheta \omega]} \\
+(8,0) T_{\left[\omega^{3}\right]}+(8,0) T_{\left[\omega^{2} \vartheta \omega\right]}+(3,2) T_{\left[\omega^{4}\right]}+ \\
(1,0) T_{\left[\omega^{10}\right]}\end{array}$ & \\
\hline & & \multirow{2}{*}{$\begin{array}{c}2 \\
\text { local }\end{array}$} & $\left(\vartheta, \frac{1}{2}\left(e_{1}+e_{3}\right)\right),\left(\omega, \frac{1}{2}\left(e_{1}+e_{3}\right)\right)$ & \multirow[b]{2}{*}{$(26,8)$} \\
\hline & & & $\begin{array}{l}(2,1) U+(2,2) T_{[\vartheta]}+(4,0) T_{[\omega]}+(2,1) T_{\left[\omega^{6}\right]}+ \\
(4,0) T_{\left[\omega^{7}\right]}+(1,0) T_{\left[\omega^{2}\right]}+(3,2) T_{[\omega \vartheta \omega]} \\
+(4,0) T_{\left[\omega^{3}\right]}+(3,2) T_{\left[\omega^{4}\right]}+(1,0) T_{\left[\omega^{10}\right]} \\
\end{array}$ & \\
\hline & & \multirow{2}{*}{$\begin{array}{c}3 \\
\text { local }\end{array}$} & $\left(\vartheta, \frac{1}{2}\left(e_{1}+e_{2}+e_{3}+e_{4}\right)\right),\left(\omega, \frac{1}{2}\left(e_{1}+e_{2}+e_{3}+e_{4}\right)\right)$ & \multirow[b]{2}{*}{$(31,7)$} \\
\hline & & & $\begin{array}{l}(2,1) U+(2,2) T_{[\vartheta]}+(4,0) T_{[\omega]}+(3,0) T_{\left[\omega^{6}\right]}+ \\
(4,0) T_{\left[\omega^{7}\right]}+(1,0) T_{\left[\omega^{2}\right]}+(3,2) T_{[\omega \vartheta \omega]} \\
+(4,0) T_{\left[\omega^{3}\right]}+(4,0) T_{\left[\omega^{2} \vartheta \omega\right]}+(3,2) T_{\left[\omega^{4}\right]}+ \\
(1,0) T_{\left[\omega^{10}\right]}\end{array}$ & \\
\hline \multirow{14}{*}{$\begin{array}{l}\Delta(54) \\
{[54,8]}\end{array}$} & \multirow[t]{4}{*}{1} & \multirow{2}{*}{$\begin{array}{c}1 \\
\text { local }\end{array}$} & $(\vartheta, 0),(\omega, 0),(\rho, 0)$ & \multirow[b]{2}{*}{$(25,1)$} \\
\hline & & & $\begin{array}{l}(1,0) U+(2,1) T_{[\vartheta]}+(9,0) T_{[\omega]}+(1,0) T_{[\rho]}+ \\
(3,0) T_{[\vartheta \rho]}+(1,0) T_{[\omega \rho]}+(1,0) T_{\left[\omega^{2} \rho\right]} \\
+(7,0) T_{\left[\omega \rho^{2} \omega^{2} \rho\right]}\end{array}$ & \\
\hline & & \multirow[t]{2}{*}{$\begin{array}{c}2 \\
\text { local }\end{array}$} & $\begin{array}{l}(\vartheta, 0),\left(\omega, \frac{1}{3}\left(e_{1}+e_{2}+2 e_{3}+2 e_{4}+e_{5}+e_{6}\right)\right) \\
\left(\rho, \frac{1}{3}\left(e_{1}+e_{2}+2 e_{3}+2 e_{4}+e_{5}+e_{6}\right)\right)\end{array}$ & \multirow[b]{2}{*}{$(13,1)$} \\
\hline & & & $\begin{array}{l}(1,0) U+(2,1) T_{[\vartheta]}+(1,0) T_{[\rho]}+(3,0) T_{[\vartheta \rho]}+ \\
(1,0) T_{[\omega \rho]}+(1,0) T_{\left[\omega^{2} \rho\right]}+(4,0) T_{\left[\omega \rho^{2} \omega^{2} \rho\right]}\end{array}$ & \\
\hline & \multirow[t]{8}{*}{2} & \multirow{2}{*}{$\begin{array}{c}1 \\
\text { local }\end{array}$} & $(\vartheta, 0),(\omega, 0),(\rho, 0)$ & \multirow[b]{2}{*}{$(25,1)$} \\
\hline & & & $\begin{array}{l}(1,0) U+(2,1) T_{[\vartheta]}+(3,0) T_{[\omega]}+(3,0) T_{[\rho]}+ \\
(3,0) T_{[\vartheta \rho]}+(3,0) T_{[\omega \rho]}+(3,0) T_{\left[\omega^{2} \rho\right]} \\
+(7,0) T_{\left[\omega \rho^{2} \omega^{2} \rho\right]}\end{array}$ & \\
\hline & & \multirow[t]{2}{*}{$\begin{array}{c}2 \\
\text { local }\end{array}$} & $\begin{array}{l}\left(\vartheta, \frac{1}{3}\left(2 e_{1}+e_{2}+e_{3}+2 e_{5}\right)\right),\left(\omega, \frac{2}{3}\left(e_{1}+e_{3}+e_{4}\right)\right), \\
\left(\rho, \frac{2}{3}\left(e_{1}+e_{3}+e_{4}\right)\right)\end{array}$ & \multirow[b]{2}{*}{$(17,1)$} \\
\hline & & & $\begin{array}{l}(1,0) U+(2,1) T_{[\vartheta]}+(3,0) T_{[\rho]}+(3,0) T_{[\vartheta \rho]}+ \\
(3,0) T_{\left[\omega^{2} \rho\right]}+(5,0) T_{\left[\omega \rho^{2} \omega^{2} \rho\right]}\end{array}$ & \\
\hline & & \multirow[t]{2}{*}{$\begin{array}{c}3 \\
\text { local }\end{array}$} & $\begin{array}{l}\left(\vartheta, \frac{1}{3}\left(2 e_{1}+2 e_{3}+e_{4}+2 e_{5}+e_{6}\right)\right),\left(\omega, \frac{1}{3}\left(2 e_{2}+\right.\right. \\
\left.\left.2 e_{3}+e_{6}\right)\right), \\
\left(\rho, \frac{1}{3}\left(2 e_{2}+2 e_{3}+e_{6}\right)\right)\end{array}$ & \multirow[b]{2}{*}{$(13,1)$} \\
\hline & & & $\begin{array}{l}(1,0) U+(2,1) T_{[\vartheta]}+(3,0) T_{[\vartheta \rho]}+(3,0) T_{\left[\omega^{2} \rho\right]}+ \\
(4,0) T_{\left[\omega \rho^{2} \omega^{2} \rho\right]}\end{array}$ & \\
\hline & & \multirow{2}{*}{$\begin{array}{c}4 \\
\text { local }\end{array}$} & $(\vartheta, 0),\left(\omega, \frac{1}{3}\left(e_{2}+e_{3}+e_{4}\right)\right),\left(\rho, \frac{1}{3}\left(e_{2}+e_{5}+2 e_{6}\right)\right)$ & \multirow[b]{2}{*}{$(9,1)$} \\
\hline & & & $\begin{array}{l}(1,0) U+(2,1) T_{[\vartheta]}+\quad+\quad(3,0) T_{[\vartheta \rho]}+ \\
(3,0) T_{\left[\omega \rho^{2} \omega^{2} \rho\right]}\end{array}$ & \\
\hline & \multirow[t]{2}{*}{3} & \multirow{2}{*}{$\begin{array}{c}1 \\
\text { local }\end{array}$} & $(\vartheta, 0),(\omega, 0),(\rho, 0)$ & \\
\hline & & & $\begin{array}{l}(1,0) U+(2,1) T_{[\vartheta]}+(3,0) T_{[\omega]}+(3,0) T_{[\rho]}+ \\
(3,0) T_{[\vartheta \rho]}+(3,0) T_{[\omega \rho]}+(3,0) T_{\left[\omega^{2} \rho\right]} \\
+(7,0) T_{\left[\omega \rho^{2} \omega^{2} \rho\right]}\end{array}$ & $(25,1)$ \\
\hline
\end{tabular}

(Table 2. Continued ...) 


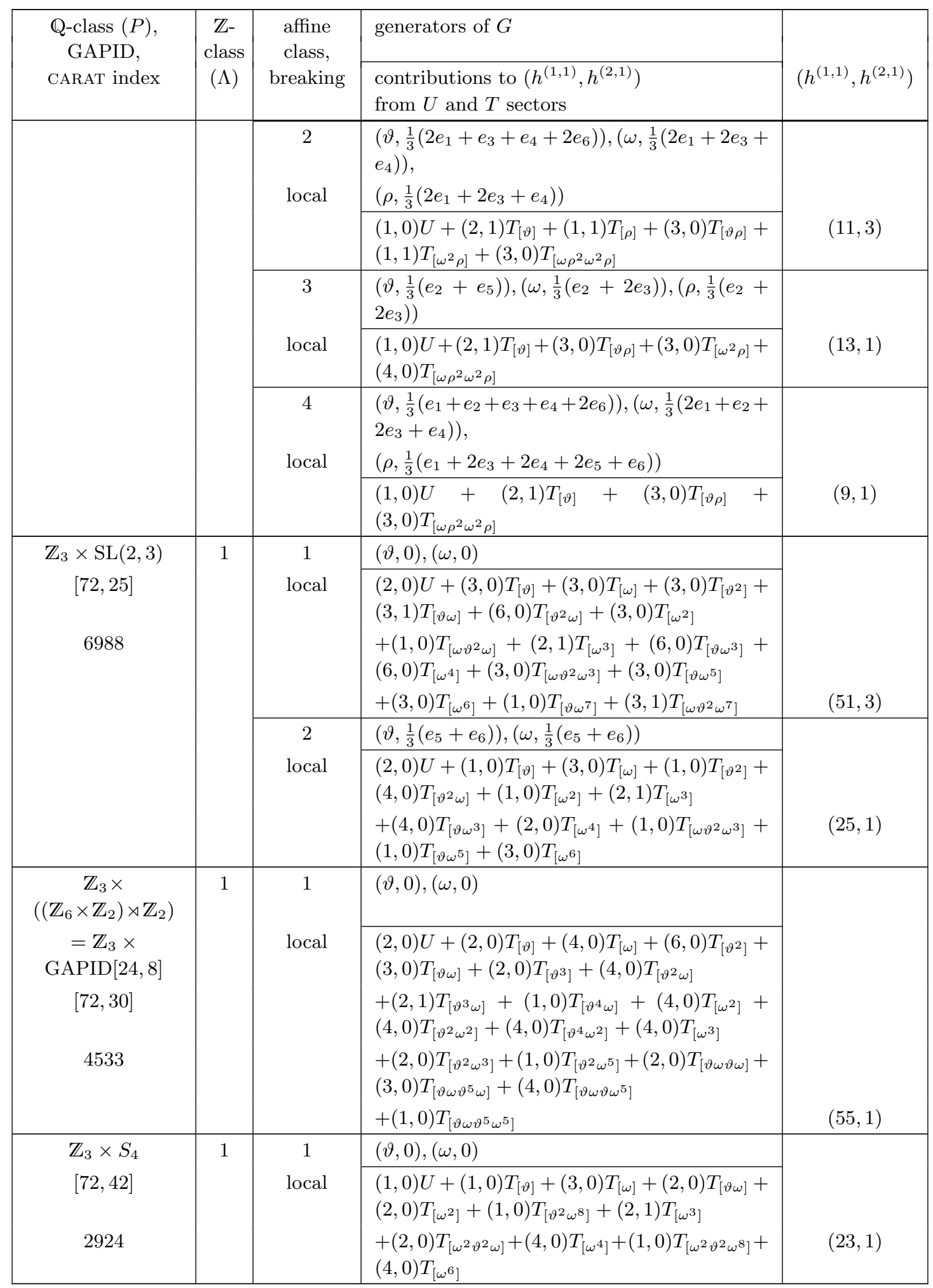

(Table 2. Continued ...) 


\begin{tabular}{|c|c|c|c|c|}
\hline \multirow{2}{*}{$\begin{array}{l}\text { Q-class }(P), \\
\text { GAPID, } \\
\text { CARAT index }\end{array}$} & \multirow{2}{*}{$\begin{array}{c}\mathbb{Z}- \\
\text { class } \\
(\Lambda)\end{array}$} & \multirow{2}{*}{$\begin{array}{l}\text { affine } \\
\text { class, } \\
\text { breaking }\end{array}$} & generators of $G$ & \multirow[b]{2}{*}{$\left(h^{(1,1)}, h^{(2,1)}\right)$} \\
\hline & & & $\begin{array}{l}\text { contributions to }\left(h^{(1,1)}, h^{(2,1)}\right) \\
\text { from } U \text { and } T \text { sectors }\end{array}$ & \\
\hline & \multirow[t]{2}{*}{2} & \multirow{2}{*}{$\begin{array}{c}1 \\
\text { local }\end{array}$} & $(\vartheta, 0),(\omega, 0)$ & \multirow[b]{2}{*}{$(20,0)$} \\
\hline & & & $\begin{array}{l}(1,0) U+(1,0) T_{[\vartheta]}+(3,0) T_{[\omega]}+(2,0) T_{[\vartheta \omega]}+ \\
(2,0) T_{\left[\omega^{2}\right]}+(1,0) T_{\left[\vartheta^{2} \omega^{8}\right]}+(1,0) T_{\left[\omega^{3}\right]} \\
+(2,0) T_{\left[\omega^{2} \vartheta^{2} \omega\right]}+(4,0) T_{\left[\omega^{4}\right]}+(1,0) T_{\left[\omega^{2} \vartheta^{2} \omega^{8}\right]}+ \\
(2,0) T_{\left[\omega^{6}\right]}\end{array}$ & \\
\hline & \multirow[t]{2}{*}{3} & \multirow{2}{*}{$\begin{array}{c}1 \\
\text { local }\end{array}$} & $(\vartheta, 0),(\omega, 0)$ & \multirow[b]{2}{*}{$(20,0)$} \\
\hline & & & $\begin{array}{l}(1,0) U+(1,0) T_{[\vartheta]}+(3,0) T_{[\omega]}+(2,0) T_{[\vartheta \omega]}+ \\
(2,0) T_{\left[\omega^{2}\right]}+(1,0) T_{\left[\vartheta^{2} \omega^{8}\right]}+(1,0) T_{\left[\omega^{3}\right]} \\
+(2,0) T_{\left[\omega^{2} \vartheta^{2} \omega\right]}+(4,0) T_{\left[\omega^{4}\right]}+(1,0) T_{\left[\omega^{2} \vartheta^{2} \omega^{8}\right]}+ \\
(2,0) T_{\left[\omega^{6}\right]}\end{array}$ & \\
\hline \multirow{18}{*}{$\begin{array}{c}\Delta(96) \\
{[96,64]}\end{array}$} & \multirow[t]{6}{*}{1} & \multirow{2}{*}{$\begin{array}{c}1 \\
\text { local }\end{array}$} & $(\vartheta, 0),(\omega, 0)$ & \multirow[b]{2}{*}{$(32,2)$} \\
\hline & & & $\begin{array}{l}(1,0) U+(1,1) T_{[\vartheta]}+(4,0) T_{[\omega]}+(3,1) T_{[\vartheta \omega]}+ \\
(9,0) T_{\left[\omega^{2}\right]}+(4,0) T_{\left[\omega^{2} \vartheta^{2} \omega\right]}+(6,0) T_{\left[\omega^{4}\right]} \\
+(4,0) T_{\left[\vartheta \omega^{3} \vartheta \omega\right]}\end{array}$ & \\
\hline & & \multirow{2}{*}{$\begin{array}{c}2 \\
\text { local }\end{array}$} & $\left(\vartheta, \frac{1}{2}\left(e_{3}+e_{4}\right)\right),\left(\omega, \frac{1}{2}\left(e_{4}+e_{5}+e_{6}\right)\right)$ & \multirow[b]{2}{*}{$(15,1)$} \\
\hline & & & $\begin{array}{l}(1,0) U+(1,1) T_{[\vartheta]}+(4,0) T_{[\omega]}+(3,0) T_{\left[\omega^{2}\right]}+ \\
(2,0) T_{\left[\omega^{2} \vartheta^{2} \omega\right]}+(4,0) T_{\left[\omega^{4}\right]}\end{array}$ & \\
\hline & & \multirow{2}{*}{$\begin{array}{c}3 \\
\text { local }\end{array}$} & $(\vartheta, 0),\left(\omega, \frac{1}{2}\left(e_{4}+e_{6}\right)\right)$ & \multirow[b]{2}{*}{$(25,1)$} \\
\hline & & & $\begin{array}{l}(1,0) U+(1,1) T_{[\vartheta]}+(4,0) T_{[\omega]}+(7,0) T_{\left[\omega^{2}\right]}+ \\
(2,0) T_{\left[\omega^{2} \vartheta^{2} \omega\right]}+(6,0) T_{\left[\omega^{4}\right]}+(4,0) T_{\left[\vartheta \omega^{3} \vartheta \omega\right]} \\
\end{array}$ & \\
\hline & \multirow[t]{6}{*}{2} & \multirow{2}{*}{$\begin{array}{c}1 \\
\text { local }\end{array}$} & $(\vartheta, 0),(\omega, 0)$ & \multirow[b]{2}{*}{$(25,1)$} \\
\hline & & & $\begin{array}{l}(1,0) U+(1,1) T_{[\vartheta]}+(4,0) T_{[\omega]}+(2,0) T_{[\vartheta \omega]}+ \\
(7,0) T_{\left[\omega^{2}\right]}+(4,0) T_{\left[\omega^{2} \vartheta^{2} \omega\right]}+(4,0) T_{\left[\omega^{4}\right]} \\
+(2,0) T_{\left[\vartheta \omega^{3} \vartheta \omega\right]}\end{array}$ & \\
\hline & & \multirow{2}{*}{$\begin{array}{c}2 \\
\text { local }\end{array}$} & $\left(\vartheta, \frac{1}{2}\left(e_{1}+e_{2}\right)\right),\left(\omega, \frac{1}{2}\left(e_{3}+e_{4}+e_{5}\right)\right)$ & \multirow[b]{2}{*}{$(17,1)$} \\
\hline & & & $\begin{array}{l}(1,0) U+(1,1) T_{[\vartheta]}+(4,0) T_{[\omega]}+(4,0) T_{\left[\omega^{2}\right]}+ \\
(2,0) T_{\left[\omega^{2} \vartheta^{2} \omega\right]}+(4,0) T_{\left[\omega^{4}\right]}+(1,0) T_{\left[\vartheta \omega^{3} \vartheta \omega\right]} \\
\end{array}$ & \\
\hline & & \multirow{2}{*}{$\begin{array}{c}3 \\
\text { local }\end{array}$} & $(\vartheta, 0),\left(\omega, \frac{1}{2}\left(e_{1}+e_{3}+e_{6}\right)\right)$ & \multirow[b]{2}{*}{$(13,1)$} \\
\hline & & & $\begin{array}{l}(1,0) U+(1,1) T_{[\vartheta]}+(4,0) T_{[\omega]}+(3,0) T_{\left[\omega^{2}\right]}+ \\
(2,0) T_{\left[\omega^{2} \vartheta^{2} \omega\right]}+(2,0) T_{\left[\omega^{4}\right]}\end{array}$ & \\
\hline & \multirow[t]{6}{*}{3} & \multirow{2}{*}{$\begin{array}{c}1 \\
\text { local }\end{array}$} & $(\vartheta, 0),(\omega, 0)$ & \multirow[b]{2}{*}{$(25,1)$} \\
\hline & & & $\begin{array}{l}(1,0) U+(1,1) T_{[\vartheta]}+(4,0) T_{[\omega]}+(2,0) T_{[\vartheta \omega]}+ \\
(7,0) T_{\left[\omega^{2}\right]}+(4,0) T_{\left[\omega^{2} \vartheta^{2} \omega\right]}+(4,0) T_{\left[\omega^{4}\right]} \\
+(2,0) T_{\left[\vartheta \omega^{3} \vartheta \omega\right]}\end{array}$ & \\
\hline & & \multirow{2}{*}{$\begin{array}{c}2 \\
\text { local }\end{array}$} & $\left(\vartheta, \frac{1}{2}\left(e_{2}+e_{3}+e_{4}\right)\right),\left(\omega, \frac{1}{2}\left(e_{4}+e_{5}+e_{6}\right)\right)$ & \\
\hline & & & $\begin{array}{l}(1,0) U+(1,1) T_{[\vartheta]}+(4,0) T_{[\omega]}+(1,1) T_{[\vartheta \omega]}+ \\
(5,0) T_{\left[\omega^{2}\right]}+(2,0) T_{\left[\omega^{2} \vartheta^{2} \omega\right]}+(4,0) T_{\left[\omega^{4}\right]} \\
+(2,0) T_{\left[\vartheta \omega^{3} \vartheta \omega\right]}\end{array}$ & $(20,2)$ \\
\hline & & \multirow{2}{*}{$\begin{array}{c}3 \\
\text { local }\end{array}$} & $(\vartheta, 0),\left(\omega, \frac{1}{2} e_{4}\right)$ & \multirow[b]{2}{*}{$(19,1)$} \\
\hline & & & $\begin{array}{l}(1,0) U+(1,1) T_{[\vartheta]}+(4,0) T_{[\omega]}+(5,0) T_{\left[\omega^{2}\right]}+ \\
(2,0) T_{\left[\omega^{2} \vartheta^{2} \omega\right]}+(4,0) T_{\left[\omega^{4}\right]}+(2,0) T_{\left[\vartheta \omega^{3} \vartheta \omega\right]}\end{array}$ & \\
\hline
\end{tabular}

(Table 2. Continued...) 


\begin{tabular}{|c|c|c|c|c|}
\hline \multirow{8}{*}{$\begin{array}{l}\text { Q-class }(P), \\
\text { GAPID, } \\
\text { CARAT index }\end{array}$} & \multirow{2}{*}{$\begin{array}{l}\mathbb{Z}- \\
\text { class } \\
(\Lambda)\end{array}$} & \multirow{2}{*}{$\begin{array}{l}\text { affine } \\
\text { class, } \\
\text { breaking }\end{array}$} & generators of $G$ & \multirow[b]{2}{*}{$\left(h^{(1,1)}, h^{(2,1)}\right)$} \\
\hline & & & $\begin{array}{l}\text { contributions to }\left(h^{(1,1)}, h^{(2,1)}\right) \\
\text { from } U \text { and } T \text { sectors }\end{array}$ & \\
\hline & \multirow[t]{6}{*}{4} & \multirow{2}{*}{$\begin{array}{c}1 \\
\text { local }\end{array}$} & $(\vartheta, 0),(\omega, 0)$ & \multirow[b]{2}{*}{$(19,1)$} \\
\hline & & & $\begin{array}{l}(1,0) U+(1,1) T_{[\vartheta]}+(4,0) T_{[\omega]}+(1,0) T_{[\vartheta \omega]}+ \\
(5,0) T_{\left[\omega^{2}\right]}+(3,0) T_{\left[\omega^{2} \vartheta^{2} \omega\right]}+(3,0) T_{\left[\omega^{4}\right]} \\
+(1,0) T_{\left[\vartheta \omega^{3} \vartheta \omega\right]}\end{array}$ & \\
\hline & & \multirow{2}{*}{$\begin{array}{c}2 \\
\text { local }\end{array}$} & $\left(\vartheta, \frac{1}{4}\left(3 e_{1}+3 e_{2}+3 e_{3}+e_{5}\right)\right),\left(\omega, \frac{1}{4}\left(3 e_{2}+e_{5}\right)\right)$ & \multirow[b]{2}{*}{$(11,2)$} \\
\hline & & & $\begin{array}{l}(1,0) U+(1,1) T_{[\vartheta]}+(4,0) T_{[\omega]}+(3,0) T_{\left[\omega^{2}\right]}+ \\
(1,1) T_{\left[\omega^{2} \vartheta^{2} \omega\right]}+(1,0) T_{\left[\omega^{4}\right]}\end{array}$ & \\
\hline & & \multirow{2}{*}{$\begin{array}{c}3 \\
\text { local }\end{array}$} & $\left(\vartheta, \frac{1}{2}\left(e_{1}+e_{2}+e_{3}+e_{5}\right)\right),\left(\omega, \frac{1}{2}\left(e_{2}+e_{5}\right)\right)$ & \\
\hline & & & $\begin{array}{l}(1,0) U+(1,1) T_{[\vartheta]}+(4,0) T_{[\omega]}+(1,0) T_{[\vartheta \omega]}+ \\
(5,0) T_{\left[\omega^{2}\right]}+(3,0) T_{\left[\omega^{2} \vartheta^{2} \omega\right]}+(3,0) T_{\left[\omega^{4}\right]} \\
+(1,0) T_{\left[\vartheta \omega^{3} \vartheta \omega\right]}\end{array}$ & $(19,1)$ \\
\hline \multirow{5}{*}{$\begin{array}{c}\mathrm{SL}(2,3) \rtimes \mathbb{Z}_{4} \\
{[96,67]}\end{array}$} & \multirow[t]{5}{*}{1} & \multirow{3}{*}{$\begin{array}{c}1 \\
\text { local }\end{array}$} & $(\vartheta, 0),(\omega, 0)$ & \multirow[b]{3}{*}{$(44,2)$} \\
\hline & & & $\begin{array}{l}(2,0) U+(3,2) T_{[\vartheta]}+(8,0) T_{[\omega]}+(4,0) T_{[\vartheta \omega]}+ \\
(2,0) T_{\left[\vartheta^{2} \omega\right]}+(6,0) T_{\left[\omega^{2}\right]}+(4,0) T_{[\omega \vartheta \omega]}\end{array}$ & \\
\hline & & & $\begin{array}{l}+(2,0) T_{\left[\omega^{2} \vartheta \omega\right]}+ \\
(3,0) T_{\left[\vartheta^{2} \omega^{3} \vartheta^{2} \omega\right]}+ \\
(3,0) T_{\left[\vartheta \omega \vartheta^{2} \omega \vartheta \omega^{2}\right]}\end{array}$ & \\
\hline & & \multirow{2}{*}{$\begin{array}{c}2 \\
\text { local }\end{array}$} & $\left(\vartheta, \frac{1}{2} e_{1}\right),\left(\omega, \frac{1}{2}\left(e_{1}+e_{2}+e_{3}\right)\right)$ & \multirow[b]{2}{*}{$(27,3)$} \\
\hline & & & $\begin{array}{l}(2,0) U+(3,2) T_{[\vartheta]}+(4,0) T_{[\omega]}+(4,0) T_{[\vartheta \omega]}+ \\
(3,0) T_{\left[\omega^{2}\right]}+(4,0) T_{[\omega \vartheta \omega]}+(1,0) T_{\left[\vartheta^{2} \omega^{3} \vartheta \omega\right]} \\
+(1,1) T_{\left[\vartheta^{2} \omega^{3} \vartheta^{2} \omega\right]}+(3,0) T_{[\vartheta \omega \vartheta \omega]}+ \\
(2,0) T_{\left[\vartheta \omega \vartheta^{2} \omega \vartheta \omega^{2}\right]}+\end{array}$ & \\
\hline \multirow{8}{*}{$\begin{array}{c}\Sigma(36 \phi) \\
{[108,15]}\end{array}$} & \multirow[t]{4}{*}{1} & \multirow{2}{*}{$\begin{array}{c}1 \\
\text { local }\end{array}$} & $(\vartheta, 0),(\omega, 0)$ & \multirow[b]{2}{*}{$(25,1)$} \\
\hline & & & $\begin{array}{l}(1,0) U+(1,0) T_{[\vartheta]}+(3,0) T_{[\omega]}+(2,1) T_{\left[\vartheta^{2}\right]}+ \\
(3,0) T_{[\vartheta \omega]}+(1,0) T_{\left[\vartheta^{3}\right]}+(3,0) T_{[\vartheta \omega \vartheta \omega]} \\
+(3,0) T_{\left[\vartheta \omega \vartheta^{3} \omega\right]}+(3,0) T_{\left[\vartheta \omega \vartheta \omega \vartheta^{3} \omega\right]}+ \\
(5,0) T_{[\vartheta \omega \vartheta \omega \vartheta \omega \vartheta \omega]}\end{array}$ & \\
\hline & & \multirow[b]{2}{*}{ local } & $\begin{array}{l}\left(\vartheta, \frac{2}{3}\left(e_{1}+e_{4}\right)\right),\left(\omega, \frac{1}{3}\left(e_{1}+2 e_{2}+2 e_{3}+e_{4}+e_{5}+\right.\right. \\
\left.\left.e_{6}\right)\right)\end{array}$ & \multirow[b]{2}{*}{$(17,1)$} \\
\hline & & & $\begin{array}{l}(1,0) U+(1,0) T_{[\vartheta]}+(2,1) T_{\left[\vartheta^{2}\right]}+(3,0) T_{[\vartheta \omega]}+ \\
(1,0) T_{\left[\vartheta^{3}\right]}+(3,0) T_{[\vartheta \omega \vartheta \omega]} \\
+(3,0) T_{\left[\vartheta \omega \vartheta \omega \vartheta^{3} \omega\right]}+(3,0) T_{[\vartheta \omega \vartheta \omega \vartheta \omega \vartheta \omega]} \\
\end{array}$ & \\
\hline & \multirow[t]{4}{*}{2} & \multirow{2}{*}{$\begin{array}{c}1 \\
\text { local }\end{array}$} & $(\vartheta, 0),(\omega, 0)$ & \multirow[b]{2}{*}{$(25,1)$} \\
\hline & & & $\begin{array}{l}(1,0) U+(1,0) T_{[\vartheta]}+(3,0) T_{[\omega]}+(2,1) T_{\left[\vartheta^{2}\right]}+ \\
(3,0) T_{[\vartheta \omega]}+(1,0) T_{\left[\vartheta^{3}\right]}+(3,0) T_{[\vartheta \omega \vartheta \omega]} \\
+(3,0) T_{\left[\vartheta \omega \vartheta^{3} \omega\right]}+\quad(3,0) T_{\left[\vartheta \omega \vartheta \omega \vartheta^{3} \omega\right]}+ \\
(5,0) T_{[\vartheta \omega \vartheta \omega \vartheta \omega \vartheta \omega]}\end{array}$ & \\
\hline & & \multirow{2}{*}{$\begin{array}{c}2 \\
\text { local }\end{array}$} & $\left(\vartheta, \frac{1}{3}\left(2 e_{3}+e_{4}\right)\right),\left(\omega, \frac{1}{3}\left(e_{1}+2 e_{4}+2 e_{5}+2 e_{6}\right)\right)$ & \multirow[b]{2}{*}{$(17,1)$} \\
\hline & & & $\begin{array}{l}(1,0) U+(1,0) T_{[\vartheta]}+(2,1) T_{\left[\vartheta^{2}\right]}+(3,0) T_{[\vartheta \omega]}+ \\
(1,0) T_{\left[\vartheta^{3}\right]}+(3,0) T_{[\vartheta \omega \vartheta \omega]} \\
+(3,0) T_{\left[\vartheta \omega \vartheta \omega \vartheta^{3} \omega\right]}+(3,0) T_{[\vartheta \omega \vartheta \omega \vartheta \omega \vartheta \omega]}\end{array}$ & \\
\hline
\end{tabular}

(Table 2. Continued...) 


\begin{tabular}{|c|c|c|c|c|}
\hline \multirow{2}{*}{$\begin{array}{c}\text { Q-class }(P), \\
\text { GAPID, } \\
\text { CARAT index }\end{array}$} & \multirow{2}{*}{$\begin{array}{c}\mathbb{Z}- \\
\text { class } \\
(\Lambda)\end{array}$} & \multirow{2}{*}{$\begin{array}{c}\text { affine } \\
\text { class, } \\
\text { breaking }\end{array}$} & generators of $G$ & \multirow[b]{2}{*}{$\left(h^{(1,1)}, h^{(2,1)}\right)$} \\
\hline & & & $\begin{array}{l}\text { contributions to }\left(h^{(1,1)}, h^{(2,1)}\right) \\
\text { from } U \text { and } T \text { sectors }\end{array}$ & \\
\hline$\Delta(108)$ & \multirow[t]{4}{*}{1} & \multirow{4}{*}{$\begin{array}{c}1 \\
\text { local }\end{array}$} & $(\vartheta, 0),(\omega, 0)$ & \multirow[b]{4}{*}{$(36,0)$} \\
\hline$[108,22]$ & & & $\begin{array}{l}(1,0) U+(1,0) T_{[\vartheta]}+(4,0) T_{[\omega]}+(1,0) T_{\left[\vartheta^{2}\right]}+ \\
(1,0) T_{[\vartheta \omega]}+(1,0) T_{\left[\vartheta^{2} \omega\right]}+(4,0) T_{\left[\omega^{2}\right]}\end{array}$ & \\
\hline \multirow[t]{2}{*}{2810} & & & $\begin{array}{l}+(1,0) T_{[\omega \vartheta \omega]}+(1,0) T_{\left[\omega \vartheta^{2} \omega\right]}+(4,0) T_{\left[\omega^{3}\right]}+ \\
(4,0) T_{\left[\omega^{4}\right]}+(2,0) T_{\left[\vartheta \omega^{4} \vartheta^{2} \omega^{5}\right]}\end{array}$ & \\
\hline & & & $\begin{array}{l}+(1,0) T_{\left[\vartheta \omega^{3} \vartheta^{2} \omega^{4}\right]}+\quad(4,0) T_{\left[\vartheta \omega^{2} \vartheta^{2} \omega^{3}\right]} \\
(1,0) T_{\left[\vartheta \omega^{4} \vartheta^{2} \omega\right]}+(5,0) T_{\left[\vartheta \omega^{2} \vartheta^{2} \omega^{4}\right]}\end{array}$ & \\
\hline \multirow{2}{*}{$\begin{array}{c}\operatorname{PSL}(3,2) \\
{[168,42]}\end{array}$} & \multirow[t]{6}{*}{1} & \multirow{2}{*}{$\begin{array}{c}1 \\
\text { local }\end{array}$} & $(\vartheta, 0),(\omega, 0)$ & \multirow[b]{2}{*}{$(14,2)$} \\
\hline & & & $\begin{array}{l}(1,0) U+(1,1) T_{[\vartheta]}+(4,0) T_{[\omega]}+(7,0) T_{[\vartheta \omega]}+ \\
(1,1) T_{\left[\vartheta^{2} \omega \vartheta \omega\right]}\end{array}$ & \\
\hline \multirow[t]{4}{*}{2934} & & \multirow{2}{*}{$\begin{array}{c}2 \\
\text { local }\end{array}$} & $(\vartheta, 0),\left(\omega, \frac{1}{2}\left(e_{3}+e_{6}\right)\right)$ & \multirow[b]{2}{*}{$(10,2)$} \\
\hline & & & $(1,0) U+(1,1) T_{[\vartheta]}+(1,1) T_{[\omega]}+(7,0) T_{[\vartheta \omega]}$ & \\
\hline & & \multirow{2}{*}{$\begin{array}{c}3 \\
\text { local }\end{array}$} & $(\vartheta, 0),\left(\omega, \frac{1}{2}\left(e_{1}+e_{2}+e_{3}+e_{5}\right)\right)$ & \multirow[b]{2}{*}{$(11,1)$} \\
\hline & & & $(1,0) U+(1,1) T_{[\vartheta]}+(2,0) T_{[\omega]}+(7,0) T_{[\vartheta \omega]}$ & \\
\hline \multirow{5}{*}{$\begin{array}{c}\Sigma(72 \phi) \\
{[216,88]}\end{array}$} & \multirow[t]{3}{*}{1} & \multirow{3}{*}{$\begin{array}{c}1 \\
\text { local }\end{array}$} & $(\vartheta, 0),(\omega, 0)$ & \multirow[b]{3}{*}{$(25,1)$} \\
\hline & & & $\begin{array}{l}(1,0) U+(1,0) T_{[\vartheta]}+(3,0) T_{[\omega]}+(2,1) T_{\left[\vartheta^{2}\right]}+ \\
(3,0) T_{[\vartheta \omega]}+(1,0) T_{\left[\vartheta^{2} \omega\right]}+(1,0) T_{\left[\vartheta^{3} \omega\right]}\end{array}$ & \\
\hline & & & $\begin{array}{l}+(3,0) T_{\left[\omega^{2}\right]}+(3,0) T_{\left[\omega \vartheta^{2} \omega\right]}+(3,0) T_{\left[\omega \vartheta^{3} \omega\right]}+ \\
(4,0) T_{\left[\omega^{4}\right]}\end{array}$ & \\
\hline & \multirow[t]{2}{*}{2} & \multirow{2}{*}{$\begin{array}{c}1 \\
\text { local }\end{array}$} & $(\vartheta, 0),(\omega, 0)$ & \multirow[b]{2}{*}{$(25,1)$} \\
\hline & & & $\begin{array}{l}(1,0) U+(1,0) T_{[\vartheta]}+(3,0) T_{[\omega]}+(2,1) T_{\left[\vartheta^{2}\right]}+ \\
(3,0) T_{[\vartheta \omega]}+(1,0) T_{\left[\vartheta^{2} \omega\right]}+(1,0) T_{\left[\vartheta^{3} \omega\right]} \\
+(3,0) T_{\left[\omega^{2}\right]}+(3,0) T_{\left[\omega \vartheta^{2} \omega\right]}+(3,0) T_{\left[\omega \vartheta^{3} \omega\right]}+ \\
(4,0) T_{\left[\omega^{4}\right]}\end{array}$ & \\
\hline$\Delta(216)$ & \multirow[t]{4}{*}{1} & \multirow{4}{*}{$\begin{array}{c}1 \\
\text { local }\end{array}$} & $(\vartheta, 0),(\omega, 0),(\rho, 0)$ & \multirow[b]{4}{*}{$(31,1)$} \\
\hline$[216,95]$ & & & $\begin{array}{l}(1,0) U+(1,0) T_{[\vartheta]}+(2,1) T_{[\omega]}+(1,0) T_{[\rho]}+ \\
(2,0) T_{[\vartheta \omega]}+(1,0) T_{[\vartheta \rho]}+(4,0) T_{\left[\vartheta^{2} \rho\right]}\end{array}$ & \\
\hline 2851 & & & $\begin{array}{l}+(3,0) T_{\left[\omega^{2}\right]}+(2,0) T_{[\omega \rho]}+(3,0) T_{\left[\vartheta^{2} \omega^{3} \rho\right]}+ \\
(1,0) T_{\left[\omega \rho \vartheta \omega^{3} \rho^{2}\right]}+(4,0) T_{\left[\omega^{2} \rho \vartheta \omega^{2} \rho\right]}\end{array}$ & \\
\hline & & & $+(4,0) T_{[\omega \rho \omega \rho]}+(2,0) T_{\left[\omega^{3} \rho \omega^{3} \rho\right]}$ & \\
\hline
\end{tabular}

Table 2. Hodge numbers of heterotic orbifolds with non-Abelian point group. 


\begin{tabular}{|c|c|c|c|c|c|}
\hline \multirow{2}{*}{$\begin{array}{c}\text { point group } P \\
\text { (Q-class) }\end{array}$} & \multirow{2}{*}{$\begin{array}{c}\mathbb{Z} \text { - and } \\
\text { affine class }\end{array}$} & \multirow{2}{*}{$\begin{array}{l}\text { Hodge numbers } \\
\left(h^{(1,1)}, h^{(2,1)}\right)\end{array}$} & \multicolumn{2}{|c|}{ origin of $\pi_{1}$ generators: } & \multirow{2}{*}{$\begin{array}{c}\text { fundamental group } \\
\pi_{1}=S /\langle F\rangle\end{array}$} \\
\hline & & & $G /\left\langle G_{F}\right\rangle$ & $\Lambda / \Lambda_{F}$ & \\
\hline \multirow[t]{3}{*}{$S_{3}$} & $1-2$ & $(6,6)$ & $\mathbb{1 1}$ & $\mathbb{Z}_{3} \times \mathbb{Z}_{3}$ & $\mathbb{Z}_{3} \times \mathbb{Z}_{3}$ \\
\hline & $2-2$ & $(6,6)$ & $\mathbb{1 1}$ & $\mathbb{Z}_{3}$ & $\mathbb{Z}_{3}$ \\
\hline & $3-2$ & $(6,6)$ & $\mathbb{1 1}$ & $\mathbb{Z}_{3}$ & $\mathbb{Z}_{3}$ \\
\hline \multirow[t]{18}{*}{$D_{4}$} & $1-3$ & $(11,11)$ & $\mathbb{Z}_{2}$ & $\mathbb{Z}^{2}$ & $\mathbb{Z}_{2} \ltimes \mathbb{Z}^{2}$ \\
\hline & $1-5$ & $(6,6)$ & $\mathbb{Z}_{2}$ & $\mathbb{Z}_{2}$ & $\mathbb{Z}_{4}$ \\
\hline & $1-6$ & $(2,2)$ & $D_{4}$ & $\mathbb{Z}^{6}$ & $S$ \\
\hline & $1-8$ & $(17,5)$ & $\mathbb{Z}_{2}$ & $\mathbb{1 1}$ & $\mathbb{Z}_{2}$ \\
\hline & $1-9$ & $(7,7)$ & $\mathbb{Z}_{2} \times \mathbb{Z}_{2}$ & $\mathbb{Z}^{2}$ & $\left(\mathbb{Z}_{2} \times \mathbb{Z}_{2}\right) \ltimes \mathbb{Z}^{2}$ \\
\hline & $2-4$ & $(9,3)$ & $\mathbb{Z}_{2}$ & $\mathbb{1}$ & $\mathbb{Z}_{2}$ \\
\hline & $2-6$ & $(4,4)$ & $\mathbb{Z}_{2}$ & $\mathbb{Z}_{2}$ & $\mathbb{Z}_{4}$ \\
\hline & $2-8$ & $(10,4)$ & $\mathbb{Z}_{2}$ & $\mathbb{1}$ & $\mathbb{Z}_{2}$ \\
\hline & $5-4$ & $(4,4)$ & $\mathbb{Z}_{2}$ & $\mathbb{Z}_{2} \times \mathbb{Z}_{2}$ & $\mathbb{Z}_{4} \times \mathbb{Z}_{2}$ \\
\hline & $5-6$ & $(12,6)$ & $\mathbb{Z}_{2}$ & $\mathbb{1 1}$ & $\mathbb{Z}_{2}$ \\
\hline & $6-3$ & $(12,6)$ & $\mathbb{1}$ & $\mathbb{Z}_{2}$ & $\mathbb{Z}_{2}$ \\
\hline & $6-4$ & $(6,6)$ & $\mathbb{Z}_{2}$ & $\mathbb{1}$ & $\mathbb{Z}_{2}$ \\
\hline & $6-6$ & $(4,4)$ & $\mathbb{Z}_{2}$ & $\mathbb{Z}_{2} \times \mathbb{Z}_{2}$ & $\mathbb{Z}_{4} \times \mathbb{Z}_{2}$ \\
\hline & $6-8$ & $(10,4)$ & $\mathbb{Z}_{2}$ & $\mathbb{Z}_{2}$ & $\mathbb{Z}_{2} \times \mathbb{Z}_{2}$ \\
\hline & $8-2$ & $(6,6)$ & $\mathbb{1}$ & $\mathbb{Z}_{2}$ & $\mathbb{Z}_{2}$ \\
\hline & $9-1$ & $(17,5)$ & $\mathbb{1 1}$ & $\mathbb{Z}_{2}$ & $\mathbb{Z}_{2}$ \\
\hline & $9-2$ & $(6,6)$ & $\mathbb{1 1}$ & $\mathbb{Z}_{2}$ & $\mathbb{Z}_{2}$ \\
\hline & $9-3$ & $(15,3)$ & 11 & $\mathbb{Z}_{2}$ & $\mathbb{Z}_{2}$ \\
\hline \multirow[t]{6}{*}{$A_{4}$} & $2-1$ & $(11,3)$ & 11 & $\mathbb{Z}_{2}$ & $\mathbb{Z}_{2}$ \\
\hline & $2-2$ & $(3,3)$ & $\mathbb{1 1}$ & $\mathbb{Z}_{4}$ & $\mathbb{Z}_{4}$ \\
\hline & $4-1$ & $(7,3)$ & $\mathbb{1 1}$ & $\mathbb{Z}_{2} \times \mathbb{Z}_{2}$ & $\mathbb{Z}_{2} \times \mathbb{Z}_{2}$ \\
\hline & $4-2$ & $(5,5)$ & $\mathbb{1 1}$ & $\mathbb{Z}_{2}$ & $\mathbb{Z}_{2}$ \\
\hline & $5-1$ & $(7,3)$ & $\mathbb{1 1}$ & $\mathbb{Z}_{2}$ & $\mathbb{Z}_{2}$ \\
\hline & $6-2$ & $(3,3)$ & $\mathbb{1}$ & $\mathbb{Z}_{2}$ & $\mathbb{Z}_{2}$ \\
\hline$Q D_{16}$ & $3-4$ & $(17,5)$ & $\mathbb{Z}_{2}$ & $\mathbb{1 1}$ & $\mathbb{Z}_{2}$ \\
\hline \multirow[t]{6}{*}{$\left(\mathbb{Z}_{4} \times \mathbb{Z}_{2}\right) \rtimes \mathbb{Z}_{2}$} & $1-11$ & $(27,3)$ & $\mathbb{Z}_{2}$ & $\mathbb{1 1}$ & $\mathbb{Z}_{2}$ \\
\hline & $1-12$ & $(15,3)$ & $\mathbb{Z}_{2}$ & $\mathbb{1 1}$ & $\mathbb{Z}_{2}$ \\
\hline & $1-18$ & $(17,5)$ & $\mathbb{Z}_{2}$ & $\mathbb{1 1}$ & $\mathbb{Z}_{2}$ \\
\hline & $1-19$ & $(15,3)$ & $\mathbb{Z}_{2}$ & $\mathbb{1 1}$ & $\mathbb{Z}_{2}$ \\
\hline & $1-21$ & $(12,6)$ & $\mathbb{Z}_{2}$ & $\mathbb{1 1}$ & $\mathbb{Z}_{2}$ \\
\hline & $1-22$ & $(10,4)$ & $\mathbb{Z}_{2} \times \mathbb{Z}_{2}$ & $\mathbb{1 1}$ & $\mathbb{Z}_{2} \times \mathbb{Z}_{2}$ \\
\hline \multirow[t]{4}{*}{$\Delta(27)$} & $1-3$ & $(12,0)$ & $\mathbb{Z}_{3}$ & $\mathbb{1 1}$ & $\mathbb{Z}_{3}$ \\
\hline & $1-4$ & $(4,0)$ & $\mathbb{Z}_{3} \times \mathbb{Z}_{3}$ & $\mathbb{1 1}$ & $\mathbb{Z}_{3} \times \mathbb{Z}_{3}$ \\
\hline & $3-3$ & $(12,0)$ & $\mathbb{Z}_{3}$ & $\mathbb{1 1}$ & $\mathbb{Z}_{3}$ \\
\hline & $3-4$ & $(4,0)$ & $\mathbb{Z}_{3} \times \mathbb{Z}_{3}$ & 11 & $\mathbb{Z}_{3} \times \mathbb{Z}_{3}$ \\
\hline
\end{tabular}

Table 3. List of all non-trivial fundamental groups for orbifolds with non-Abelian $P$. The first column specifies $P$ and the second column enumerates the respective $\mathbb{Z}$ - and affine classes. In the third column we list the Hodge numbers in order to identify those cases which allow for chiral spectra, c.f. [54]. The forth and fifth column help to identify the origin of the generators of $\pi_{1}$ from the orbifolding group $G$ and from the lattice $\Lambda$, respectively. Finally, the last column lists $\pi_{1}$. 
Open Access. This article is distributed under the terms of the Creative Commons Attribution License which permits any use, distribution and reproduction in any medium, provided the original author(s) and source are credited.

\section{References}

[1] L.J. Dixon, J.A. Harvey, C. Vafa and E. Witten, Strings on orbifolds, Nucl. Phys. B 261 (1985) 678 [INSPIRE].

[2] L.J. Dixon, J.A. Harvey, C. Vafa and E. Witten, Strings on orbifolds. 2, Nucl. Phys. B 274 (1986) 285 [INSPIRE].

[3] A.E. Faraggi, A new standard-like model in the four-dimensional free fermionic string formulation, Phys. Lett. B 278 (1992) 131 [INSPIRE].

[4] T. Dijkstra, L. Huiszoon and A. Schellekens, Supersymmetric standard model spectra from RCFT orientifolds, Nucl. Phys. B 710 (2005) 3 [hep-th/0411129] [INSPIRE].

[5] F. Gmeiner, R. Blumenhagen, G. Honecker, D. Lüst and T. Weigand, One in a billion: MSSM-like D-brane statistics, JHEP 01 (2006) 004 [hep-th/0510170] [INSPIRE].

[6] R. Blumenhagen, B. Körs, D. Lüst and S. Stieberger, Four-dimensional string compactifications with D-branes, orientifolds and fluxes, Phys. Rept. 445 (2007) 1 [hep-th/0610327] [INSPIRE].

[7] F. Gmeiner and G. Honecker, Millions of standard models on $Z_{6}^{\prime}$ ?, JHEP 07 (2008) 052 [arXiv:0806.3039] [INSPIRE].

[8] B.S. Acharya, K. Bobkov, G.L. Kane, J. Shao and P. Kumar, The $G_{2}$-MSSM: an M-theory motivated model of particle physics, Phys. Rev. D 78 (2008) 065038 [arXiv:0801.0478] [INSPIRE].

[9] V. Bouchard and R. Donagi, An SU(5) heterotic standard model, Phys. Lett. B 633 (2006) 783 [hep-th/0512149] [INSPIRE].

[10] L.B. Anderson, J. Gray, A. Lukas and E. Palti, Two hundred heterotic standard models on smooth Calabi-Yau threefolds, Phys. Rev. D 84 (2011) 106005 [arXiv:1106.4804] [InSPIRE].

[11] M. Blaszczyk, S. Nibbelink Groot, F. Ruehle, M. Trapletti and P.K. Vaudrevange, Heterotic MSSM on a resolved orbifold, JHEP 09 (2010) 065 [arXiv: 1007.0203] [INSPIRE].

[12] M. Blaszczyk, N.G. Cabo Bizet, H.P. Nilles and F. Ruhle, A perfect match of MSSM-like orbifold and resolution models via anomalies, JHEP 10 (2011) 117 [arXiv:1108.0667] [INSPIRE].

[13] M. Blaszczyk, S. Groot Nibbelink and F. Ruehle, Gauged linear $\sigma$-models for toroidal orbifold resolutions, JHEP 05 (2012) 053 [arXiv: 1111.5852] [INSPIRE].

[14] W. Buchmüller, J. Louis, J. Schmidt and R. Valandro, Voisin-Borcea manifolds and heterotic orbifold models, JHEP 10 (2012) 114 [arXiv:1208.0704] [INSPIRE].

[15] W. Buchmüller, K. Hamaguchi, O. Lebedev and M. Ratz, Supersymmetric standard model from the heterotic string, Phys. Rev. Lett. 96 (2006) 121602 [hep-ph/0511035] [INSPIRE].

[16] W. Buchmüller, K. Hamaguchi, O. Lebedev and M. Ratz, Supersymmetric standard model from the heterotic string (II), Nucl. Phys. B 785 (2007) 149 [hep-th/0606187] [INSPIRE]. 
[17] O. Lebedev et al., A mini-landscape of exact MSSM spectra in heterotic orbifolds, Phys. Lett. B 645 (2007) 88 [hep-th/0611095] [INSPIRE].

[18] O. Lebedev, H.P. Nilles, S. Ramos-Sanchez, M. Ratz and P.K. Vaudrevange, Heterotic mini-landscape. (II). Completing the search for MSSM vacua in a $Z_{6}$ orbifold, Phys. Lett. B 668 (2008) 331 [arXiv:0807.4384] [INSPIRE].

[19] O. Lebedev and S. Ramos-Sanchez, The NMSSM and string theory, Phys. Lett. B 684 (2010) 48 [arXiv: 0912.0477] [INSPIRE].

[20] I. Antoniadis, E. Gava, K. Narain and T. Taylor, Effective $\mu$ term in superstring theory, Nucl. Phys. B 432 (1994) 187 [hep-th/9405024] [InSPIRE].

[21] R. Kappl et al., Large hierarchies from approximate R symmetries, Phys. Rev. Lett. 102 (2009) 121602 [arXiv:0812.2120] [INSPIRE].

[22] T. Kobayashi, H.P. Nilles, F. Plöger, S. Raby and M. Ratz, Stringy origin of non-abelian discrete flavor symmetries, Nucl. Phys. B 768 (2007) 135 [hep-ph/0611020] [INSPIRE].

[23] H.M. Lee et al., A unique $Z_{4}^{R}$ symmetry for the MSSM, Phys. Lett. B 694 (2011) 491 [arXiv: 1009.0905] [INSPIRE].

[24] S. Förste, H.P. Nilles, S. Ramos-Sánchez and P.K. Vaudrevange, Proton hexality in local grand unification, Phys. Lett. B 693 (2010) 386 [arXiv:1007.3915] [INSPIRE].

[25] T. Kobayashi and N. Ohtsubo, Geometrical aspects of $Z_{N}$ orbifold phenomenology, Int. J. Mod. Phys. A 9 (1994) 87 [inSPIRE].

[26] D. Bailin and A. Love, Orbifold compactifications of string theory, Phys. Rept. 315 (1999) 285 [INSPIRE].

[27] R. Blumenhagen and E. Plauschinn, Intersecting D-branes on shift $Z_{2} \times Z_{2}$ orientifolds, JHEP 08 (2006) 031 [hep-th/0604033] [INSPIRE].

[28] R. Donagi and K. Wendland, On orbifolds and free fermion constructions, J. Geom. Phys. 59 (2009) 942 [arXiv: 0809.0330] [inSPIRE].

[29] R. Donagi and A.E. Faraggi, On the number of chiral generations in $Z_{2} \times Z_{2}$ orbifolds, Nucl. Phys. B 694 (2004) 187 [hep-th/0403272] [INSPIRE].

[30] S. Förste, T. Kobayashi, H. Ohki and K.-j. Takahashi, Non-factorisable $Z_{2} \times Z_{2}$ heterotic orbifold models and Yukawa couplings, JHEP 03 (2007) 011 [hep-th/0612044] [INSPIRE].

[31] F. Beye, T. Kobayashi and S. Kuwakino, Gauge symmetries in heterotic asymmetric orbifolds, arXiv:1304.5621 [INSPIRE].

[32] M. Fischer, M. Ratz, J. Torrado and P.K. Vaudrevange, Classification of symmetric toroidal orbifolds, JHEP 01 (2013) 084 [arXiv:1209.3906] [INSPIRE].

[33] Z. Kakushadze, G. Shiu and S.H. Tye, Asymmetric non-abelian orbifolds and model building, Phys. Rev. D 54 (1996) 7545 [hep-th/9607137] [INSPIRE].

[34] S.J. Konopka, Non abelian orbifold compactifications of the heterotic string, arXiv: 1210.5040 [INSPIRE].

[35] G. Ross, Wilson line breaking and gauge coupling unification, hep-ph/0411057 [INSPIRE].

[36] A. Hebecker and M. Trapletti, Gauge unification in highly anisotropic string compactifications, Nucl. Phys. B 713 (2005) 173 [hep-th/0411131] [INSPIRE]. 
[37] A. Anandakrishnan and S. Raby, SU(6) GUT breaking on a projective plane, Nucl. Phys. B 868 (2013) 627 [arXiv:1205.1228] [INSPIRE].

[38] T. Kobayashi, S. Raby and R.-J. Zhang, Searching for realistic 4d string models with a Pati-Salam symmetry: orbifold grand unified theories from heterotic string compactification on a $Z_{6}$ orbifold, Nucl. Phys. B 704 (2005) 3 [hep-ph/0409098] [INSPIRE].

[39] J.E. Kim, J.-H. Kim and B. Kyae, Superstring standard model from $Z_{12-I}$ orbifold compactification with and without exotics and effective R-parity, JHEP 06 (2007) 034 [hep-ph/0702278] [INSPIRE].

[40] M. Blaszczyk et al., A $Z_{2} \times Z_{2}$ standard model, Phys. Lett. B 683 (2010) 340 [arXiv: 0911.4905] [INSPIRE].

[41] D.K.M. Pena, H.P. Nilles and P.-K. Oehlmann, A zip-code for quarks, leptons and Higgs bosons, JHEP 12 (2012) 024 [arXiv:1209.6041] [INSPIRE].

[42] L.E. Ibáñez, J. Mas, H.-P. Nilles and F. Quevedo, Heterotic strings in symmetric and asymmetric orbifold backgrounds, Nucl. Phys. B 301 (1988) 157 [INSPIRE].

[43] J. Casas, A. de la Macorra, M. Mondragón and C. Muñoz, $Z_{7}$ phenomenology, Phys. Lett. B 247 (1990) 50 [INSPIRE].

[44] H. Brown et al., Crystallographic groups of four-dimensional space, J. Wiley \& Sons, U.S.A. (1978).

[45] J. Opgenorth, W. Plesken, and T. Schulz, Cristallographic algorithms and tables, Acta Cryst. Sect. A 54 (1998).

[46] The GAP Group, GAP - Groups, Algorithms, and Programming, version 4.6.3, (2013).

[47] J.R. Ellis, A. Lahanas, D.V. Nanopoulos and K. Tamvakis, No-scale supersymmetric standard model, Phys. Lett. B 134 (1984) 429 [INSPIRE].

[48] M. Cvetič, J. Louis and B.A. Ovrut, A string calculation of the Kähler potentials for moduli of $Z_{N}$ orbifolds, Phys. Lett. B 206 (1988) 227 [INSPIRE].

[49] A. Brignole, L.E. Ibáñez and C. Muñoz, Soft supersymmetry breaking terms from supergravity and superstring models, hep-ph/9707209 [INSPIRE].

[50] L. Covi et al., De Sitter vacua in no-scale supergravities and Calabi-Yau string models, JHEP 06 (2008) 057 [arXiv: 0804.1073] [INSPIRE].

[51] L.E. Ibáñez and D. Lüst, Duality anomaly cancellation, minimal string unification and the effective low-energy Lagrangian of $4 D$ strings, Nucl. Phys. B 382 (1992) 305 [hep-th/9202046] [inSPIRE].

[52] M. Dine, N. Seiberg, X. Wen and E. Witten, Nonperturbative effects on the string world sheet, Nucl. Phys. B 278 (1986) 769 [INSPIRE].

[53] L.J. Dixon, V. Kaplunovsky and J. Louis, On effective field theories describing $(2,2)$ vacua of the heterotic string, Nucl. Phys. B 329 (1990) 27 [InSPIRE].

[54] S. Groot Nibbelink and P.K.S. Vaudrevange, Schoen manifold with line bundles as resolved magnetized orbifolds, JHEP 03 (2013) 142 [arXiv: 1212.4033] [INSPIRE].

[55] H.P. Nilles, S. Ramos-Sanchez, P.K.S. Vaudrevange and A. Wingerter, The orbifolder: a tool to study the low energy effective theory of heterotic orbifolds, Comput. Phys. Commun. 183 (2012) 1363 [arXiv: 1110.5229] [inSPIRE]. 
[56] L.E. Ibáñez, H.P. Nilles and F. Quevedo, Orbifolds and Wilson lines, Phys. Lett. B 187 (1987) 25 [INSPIRE].

[57] R. Brown and P.J. Higgins, The fundamental groupoid of the quotient of a Hausdorff space by a discontinuous action of a discrete group is the orbit groupoid of the induced action, math/0212271.

[58] L.B. Anderson, J. Gray, Y.-H. He and A. Lukas, Exploring positive monad bundles and a new heterotic standard model, JHEP 02 (2010) 054 [arXiv:0911.1569] [INSPIRE].

[59] V. Braun, On free quotients of complete intersection Calabi-Yau manifolds, JHEP 04 (2011) 005 [arXiv: 1003.3235] [INSPIRE].

[60] E. Witten, Strong coupling expansion of Calabi-Yau compactification, Nucl. Phys. B 471 (1996) 135 [hep-th/9602070] [INSPIRE].

[61] H. Abe, K.-S. Choi, T. Kobayashi and H. Ohki, Magnetic flux, Wilson line and orbifold, Phys. Rev. D 80 (2009) 126006 [arXiv:0907.5274] [InSPIRE].

[62] A.-K. Kashani-Poor, R. Minasian and H. Triendl, Enhanced supersymmetry from vanishing Euler number, JHEP 04 (2013) 058 [arXiv:1301.5031] [INSPIRE].

[63] H. Ishimori et al., An introduction to non-abelian discrete symmetries for particle physicists, Lect. Notes Phys. 858 (2012) 1.

[64] C. Luhn, S. Nasri and P. Ramond, Tri-bimaximal neutrino mixing and the family symmetry semidirect product of $Z_{7}$ and $Z_{3}$, Phys. Lett. B 652 (2007) 27 [arXiv:0706.2341] [InSPIRE].

[65] C. Luhn, K.M. Parattu and A. Wingerter, A minimal model of neutrino flavor, JHEP 12 (2012) 096 [arXiv:1210.1197] [INSPIRE].

[66] R. Blumenhagen, L. Görlich and B. Körs, Supersymmetric 4D orientifolds of type IIA with D6-branes at angles, JHEP 01 (2000) 040 [hep-th/9912204] [INSPIRE].

[67] S. Förste, G. Honecker and R. Schreyer, Supersymmetric $Z_{N} \times Z_{M}$ orientifolds in $4 D$ with D-branes at angles, Nucl. Phys. B 593 (2001) 127 [hep-th/0008250] [INSPIRE].

[68] J. Erler, Anomaly cancellation in six-dimensions, J. Math. Phys. 35 (1994) 1819 [hep-th/9304104] [INSPIRE].

[69] G. Honecker and M. Trapletti, Merging heterotic orbifolds and K3 compactifications with line bundles, JHEP 01 (2007) 051 [hep-th/0612030] [INSPIRE]. 